\title{
PRIMARY PRODUCTIVITY BY PHYTOPLANKTON IN THE TIDAL, FRESH POTOMAC RIVER, MARYLAND, MAY 1980 TO AUGUST 1981
}

By Ronald R. H. Cohen and Sheryl O. Pollock 


\section{UNITED STATES DEPARTMENT OF THE INTERIOR WILLIAM P. CLARK, Secretary}

GEOLOGICAL SURVEY

Dallas L. Peck, Director

For additional information write to:

Chief Hydrologist U.S. Geological Survey 430 National Center Reston, Virginia 22092
Copies of this report can be purchased from:

U.S. Geological Survey Open-File Services Section Western Distribution Branch Box 25425, Federal Center Denver, Colorado 80225 
Errata: Primary Productivity by Phytoplankton in the tidal fresh Potomac River, Maryland, May 1980 to August 1981. R.R.H. Cohen and S.0. Pollock

Page 18, Table 2 - the units for $\mathrm{p}_{\max }$ should be changed from mg $\mathrm{O}_{2} \mathrm{~L}^{-1} \mathrm{D}-1$, to $\mathrm{O}_{2} \mathrm{~L}^{-1} \mathrm{H}^{-1}$ per mg Chlorophy11-a

Page 26, Table 7 - fourth line of legend, "Hallowing is Hollowing Point" should read "Hal is Hallowing Point" 


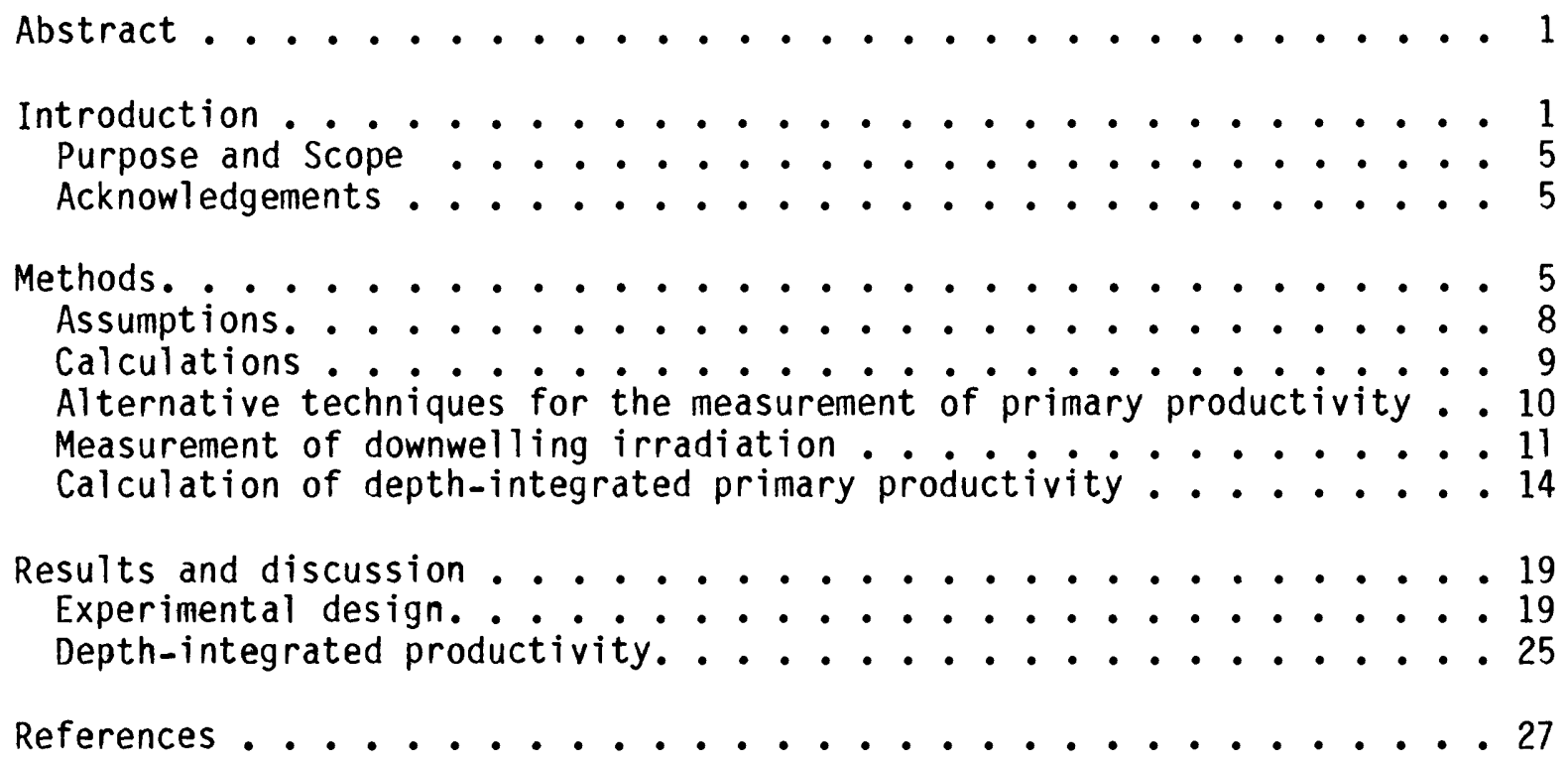




\section{ILLUSTRATIONS}

Figure 1. Map of tidal Potomac River and Estuary . . . . . . . . 2

2. Map of fresh, tidal Potomac River ........... 3

3. Diagram of the dock-side incubator used for productivity incubations. ................... 7

4. Graph showing the relationship of extinction coefficients calculated from Secchi depth, y, to those determined using a light meter that measures light in the radiation band that is useful for photosynthesis . . . . . . . . 12

5. Examples of gross primary productivity, GPP, and respiration, R, as a function of depth ............ 15

6. Graph of gross primary productivity as a function of light intensity. ................... 17

7. Flow chart for the measurement and calculation of primary productivity ................ 23

\section{TABLES}

Table 1. Solar radiation extinction coefficients $(K)$ in the water column in the fresh, tidal Potomac River.......... 13

2. The maximum rate of primary productivity, $P_{\max }$, and the half-saturation constant, $K_{m}$, in the fresh tidal Potomac River, Maryland from May 1980 to August 1981. . . . . . 18

3. Productivity measurements on samples collected through a hose and pump, and on samples incubated in the water column compared to samples collected with a depth-integrated bottle and incubated in an incubation box........... 20

4. Productivity of samples incubated in 300-milliliter bottles compared to 1-liter bottles, point samples compared to depth-integrated samples, and samples incubated for 10-hours compared to 4-hours ................. 21

5. Depth-integrated gross primary productivity determined by the graphical method, numeric integration, Talling's method, and the modified Talling method ........... 24

6. Productivity and chlorophyll a data from May 1980 to September 1981

7. Depth-integrated primary productivity in the tidal, freshwater Potomac River, Md. ............... 26 
FACTORS FOR CONVERTING INTERNATIONAL SYSTEM OF UNITS (SI)

TO INCH-POUND UNITS

Multiply SI unit

meter ( $m$ )

kilometer $(\mathrm{km})$

kilometer $(\mathrm{km})$

gram $(\mathrm{g})$

cubic meter per second $\left(\mathrm{m}^{3} \mathrm{~s}^{-1}\right)$
By

$$
\begin{aligned}
& 3.281 \\
& 0.6214 \\
& 0.5400 \\
& 0.0022 \\
& 35.31
\end{aligned}
$$

To obtain inch-pound unit

foot
mile
nautical mile
pound
cubic foot per second

Concentration Conversions

Constituent

Nitrate

Ammonia

Phosphate
From

micromoles per liter

micromoles per liter

micromoles per liter
To

milligrams per liter (N) milligrams per liter (N) milligrams per liter (N)
Divide by

0.0140

0.0140

0.0310 
PRIMARY PRODUCTIVITY BY PHYTOPLANKTON IN THE TIDAL, FRESH POTOMAC RIVER, MARYLAND, MAY 1980 TO AUGUST 1981

by Ronald R. H. Cohen and Sheryl 0. Pollock

\section{ABSTRACT}

Primary productivity, the assimilation of carbon and evolution of oxygen by phytoplankton, was measured on samples collected from the tidal Potomac River, Maryland. The studies were performed monthly from May 1980 through September 1981. Additional studies were done once a week in August 1980, twice a week from August 4 through 8, 1980, and twice in September 1980. Depth-integrated samples were collected at five stations and incubated in boxes that were exposed to natural sunlight. The boxes were covered with neutral density filters transmitting 100-, 65-, 32-, 16- and 6-percent surface light. River water was pumped continuously over the samples. The extinction of light in the water column was measured when samples were collected.

Methods for routine productivity analysis were evaluated. No difference was found between productivity (1) determined in-situ and in the boxes; (2) measured in 300-milliliter and 1-liter B.0.D. bottTes; (3) measured in point samples and depth integrated samples; and (4) calculated from short term ( 4 hours) and long term (10-24 hours) incubations. Productivity in samples that were rotated among different light intensities every 15 minutes (simulating mixing) was higher than those in bottles that remained stationary. Respiration was significantly less in samples pumped through a hose from those collected using a depth-integrating sampler.

Depth-integrated primary productivity was determined from the productivity data using an equation modified from one reported in the literature. Depth-integrated gross primary productivity was highest in August 1980 and 1981 and lowest in January 1980.

\section{INTRODUCTION}

The tidal Potomac River and Estuary, Md. extends 187 kilometers from above Washington, D.C. at Chain Bridge to the Chesapeake Bay (fig. 1). The tidal, fresh-water reach, approximately $62 \mathrm{~km}$ long, has a volume of $3.4 \mathrm{x}$ $10^{8} \mathrm{~m}^{3}$ and receives drainage from the non-tidal Potomac River and metropolitan Washington, D.C. It has an average flow of $310 \mathrm{~m}^{3} \mathrm{sec}^{-1}$ and receives approximately $1.4 \times 10^{6} \mathrm{~m}^{3}$ per day of treated waste water from municipal treatment facilities. During the summer, a zone of high phytoplankton concentration extends from river kilometer 180 at Memorial Bridge to kilometer 126 at Quantico, Va., the approximate, late-summer location of the brackish-freshwater interface (fig. 2). 


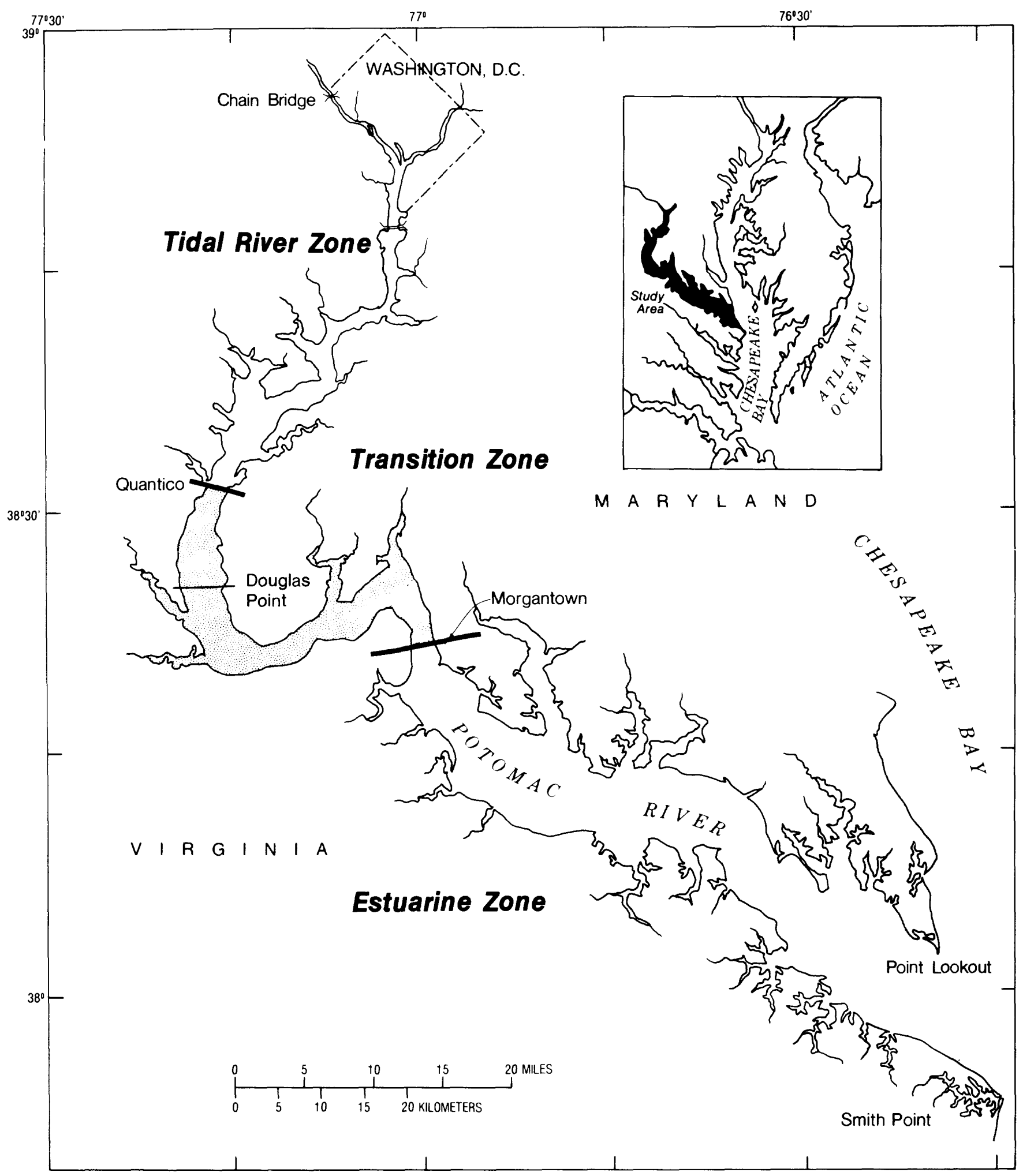

Figure 1.--Tidal Potomac River and Estuary. Shaded area is the transition zone between fresh, tidal river and brackish estuary. Thick lines delineate approximate boundaries of zones. 


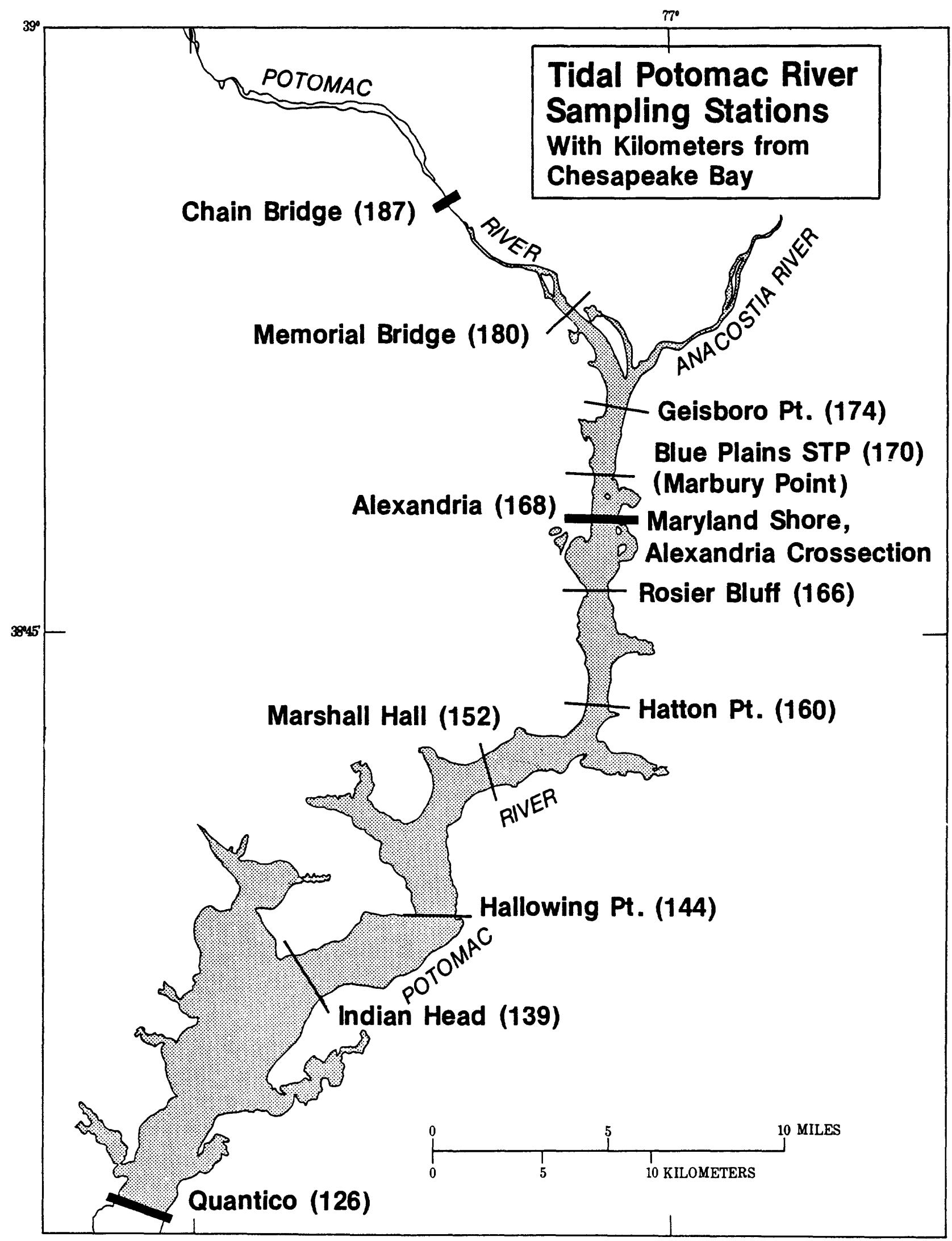

Figure 2.--The fresh, tidal Potomac River. Thick lines identify sites of cross sections that were sampled frequently as part of the Potomac River transport study. The thin lines identify sites of cross sections that were sampled in less detail or in special studies. 
Phytoplankton produce organic compounds from inorganic nutrients using light as an energy source. The organic material formed by the phytoplankton is called "primary production" and primary production per unit time per volume of water (or under a unit surface area), is called "primary productivity". The process by which phytoplankton synthesize organic material, photosynthesis, consumes inorganic carbon and releases oxygen. Thus, a measurement of the rate of increase of oxygen in solution due to the phytoplankton is a direct measurement of primary productivity.

Primary productivity measurements can yield valuable information about aquatic ecosystems. The ratio of the moles of carbon dioxide taken up to the moles of oxygen evolved by phytoplankton (the phytosynthetic quotient) is approximately 1.0 (Parsons and others, 1977). Therefore, primary productivity measurements are estimates of the increase of the carbon biomass of phytoplankton--an indication of the rate of growth. The effects of light, temperature, nutrients, and toxins on phytoplankton growth can be determined from primary productivity measurements. The information can be used to estimate the contribution of phytoplankton to the diel dissolved oxygen regimes of aquatic systems.

Sampling stations for productivity analysis or productivity experiments are listed below (with the depth used to calculate depth-integrated productivity) and shown in figure 2. (Potomac River at Douglas Point is shown in fig. 1.) River distances are measured from the center of a line drawn between Smith Point and Point Lookout at the mouth of the Potomac River.

\begin{tabular}{|c|c|c|c|}
\hline Station number & Station name & $\begin{array}{l}\text { River Distance } \\
\text { from Chesapeake } \\
\text { Bay, in kilometers }\end{array}$ & $\begin{array}{l}\text { Depth, ir } \\
\text { meters }\end{array}$ \\
\hline 01652590 & $\begin{array}{l}\text { Potomac River at } \\
\text { Alexandria, Va. } \\
\text { (in the channel near } \\
\text { the Virginia shoreline) }\end{array}$ & 168 & 2.2 \\
\hline 384736077013300 & $\begin{array}{l}\text { Potomac River across } \\
\text { from Alexandria, Va., } \\
\text { near Maryland shoreline }\end{array}$ & 168 & 2.8 \\
\hline 384318077020300 & $\begin{array}{l}\text { Potomac River at } \\
\text { Hatton Point, Md. }\end{array}$ & 160 & 3.9 \\
\hline 384136077054600 & $\begin{array}{l}\text { Potomac River at } \\
\text { Marshall Hall } \\
\text { (Mt. Vernon), Va. }\end{array}$ & 152 & 3.7 \\
\hline 383818077072800 & $\begin{array}{l}\text { Potomac River at } \\
\text { Hallowing Point, Va. }\end{array}$ & 144 & 5.6 \\
\hline
\end{tabular}


Station number

01658710

382640077159900
Station name

Potomac River at Quantico, Va.

Potomac River at Douglas Point, Md.
River Distance

from Chesapeake

Bay, in kilometers
Depth, in meters

126

5.8

117

3.8

\section{Purpose and Scope}

The purpose of this study was to determine primary productivity and respiration of the fresh, tidal Potomac River. The data will be used by the authors and other researchers involved in modelling chemical and biological properties to calculate phytoplankton growth rates. Methods are documented in detail so that the report could serve as a reference.

The report covers the period from May 1980 through August 1981. Five stations were selected for study that are representative of biologically important reaches of the fresh, tidal Potomac River.

\section{Acknowledgements}

Joan Woodward performed the chlorophyll a analyses from May 1980 through April 1981. Sheryl 0. Pollock performed chlorophyll a analyses from May through September 1981.

\section{METHODS}

Measurement of primary productivity poses many problems in methodology (Berman and Eppley, 1974). Some of the difficulties include:

1) Large surface area to volume ratio in bottles offers colonization substrate for bacteria. The bacteria increase respiration rates and remove nutrients from the water.

2) Sedimentation during incubation increases self-shading by phytoplankton.

3) Incubation at fixed depths or light intensities induces inhibition of productivity at high light levels in phytoplankton that are adapted to mixing (Marra, 1978).

4) Pump sampling of phytoplankton may result in cell damage and aberrations in productivity capacity.

5) All difficulties increase with the length of the incubation. 
Therefore, several alternative procedures for measuring primary productivity were considered and tested. We examined the differences due to methods of sample collection and the volume, length of time and technique of incubation. The methods chosen for primary productivity measurements were selected on the basis of precision, accuracy, and available resources such as research ships and personnel.

We used a light - and dark-bottle oxygen method for determining phytoplankton productivity similar to that described by Greeson and others (1977). Depth-integrated samples were collected with an open, weighted, 4-liter, polyethylene bottle fitted with a vent tube. The bottle was filled as it was lowered and raised in the water column at a uniform rate. Samples were composited until a 20-liter, polyethylene carboy was filled. At the Quantico station, two verticals were depth integrated and composited. The samples were collected during the evening (between 1700 and 2100 hours) and incubated overnight and throughout the next day, for a total of 24 hours. The first two productivity determinations (May 1980) were performed from dawn to dusk. Dawn to dusk, 4-hour and 2-hour, mid-day productivity incubations have been recommended (Vollenweider, 1965). However, nutrients for nutrient limitation bioassays must be added during the evening to demonstrate any significant stimulation during the next day (Stross, 1980).

In August and September 1980, samples were often supersaturated with oxygen at the time of collection. The 20-1iter carboy was shaken until oxygen decreased to saturation concentration. To delay oxygen supersaturation in August and September 1981, oxygen was purged from the carboy by bubbling nitrogen gas through perforated, polyethylene tubes until dissolved oxygen levels were $4-5 \mathrm{mg} \mathrm{L}^{-1}$. In spite of these precautions, oxygen degassing occurred in bottles exposed to full sunlight by 1300 to 1400 hours on some clear days in August and September. As soon as small bubbles were observed in several bottles, dissolved oxygen was measured in all the bottles for that station.

Samples from Alexandria stations were placed in incubation bottles and were incubating typically within 30-45 minutes of sampling. Samples from stations more distant from the incubation site required delays of 1-2 hours. Clear and opaque, black, 300-mL B.0.D. bottles were filled by siphon from the 20-liter bottles. Additional nutrients for bioassays were added to nine bottles. One milliliter of 18.7 millimole ammonium chloride was added to three bottles and 1 milliliter of 4.12 millimole sodium nitrate was added to another three to yield minimum concentrations of 0.02 and $0.01 \mathrm{millimole} \mathrm{NH}_{4}$ as ammonia, and $\mathrm{NO}_{3}$ as nitrate, respectively. One milliliter of $0.74 \mathrm{milli-}$ mole potassium phosphate was added to three bottles such that the concentration of phosphate attained a minimum of 0.002 millimole as $\mathrm{PO}_{4}$. Prior to incubation, dissolved oxygen was measured in all the bottles with an Orbisphere 1 / polarographic oxygen probe; the B.0.D. bottles were then sealed.

The bottles were placed in $92-\mathrm{cm}$ wide by $122-\mathrm{cm}$ long by $15-\mathrm{cm}-\mathrm{high}$ wooden boxes that were placed on a dock (fig. 3). The boxes were filled to overflowing with river water by submersible pumps capable of $0.003 \mathrm{~m} / \mathrm{s}$. Thus, the bottles were maintained at in situ river temperatures. The boxes 


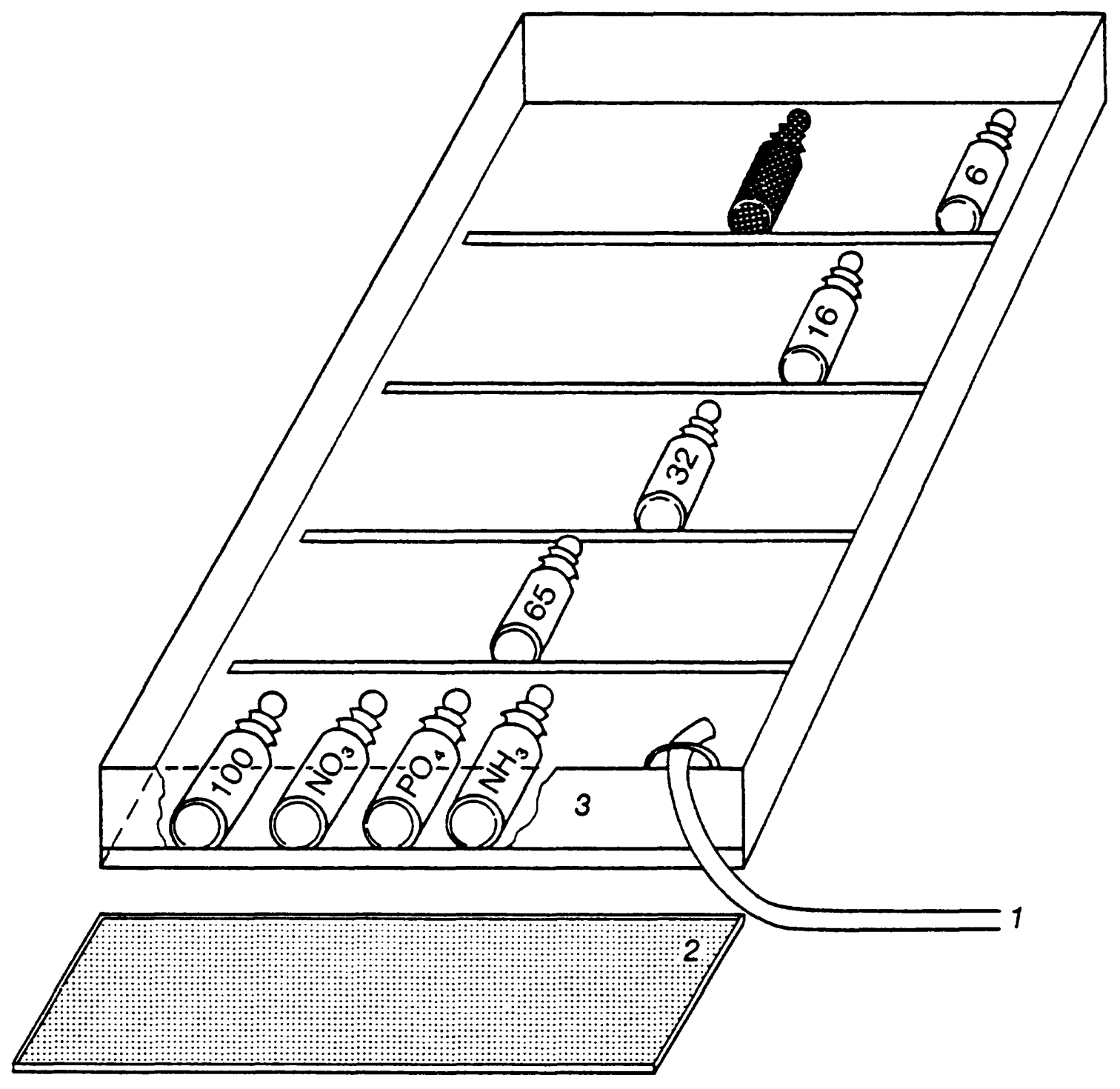

Figure 3.--Diagram of dock-side incubator used for productivity incubations: 1 is a hose from which river water flows into the incubation box; 2 is a nylon-mesh screen; 3 is the incubation box; 100, 65, 32, 16,6 are the percentages of surface light intensity as regulated by the number of screens; $\mathrm{NO}_{3}, \mathrm{PO}_{4}, \mathrm{NH}_{3}$ are nitrate-, phosphateand ammonia-enriched samples incubated under 100 percent light. The dark bottle is incubated under 6 percent light.

$$
\text { - } 7 \text { - }
$$


were divided into five sections by 2-cm-high partitions. One section was exposed to full, surface sunlight. The other sections were covered by 1, 2, 3 , or 5 layers of nylon screen transmitting 65-, 32-, 16-, and 6-percent surface light, respectively.

A black tarpaulin covered the boxes from the beginning of the incubation (in the evening) until just before dawn. Three clear bottles were placed in full sunlight and two were placed in box sections exposed to 65-, 32-, 16-, and 6-percent of full sunlight. Bottles spiked with nutrients were placed in full sunlight. Five black bottles were placed under the five layers of screen. Bottles were shaken and rotated every hour to eliminate artifacts due to settling phytoplankton and sediment. Dissolved oxygen was measured at the end of the incubation. Samples for chlorophyll-a analysis and cell enumeration and indentification were taken at the beginning and end of the incubations. The chlorophyll-a concentrations were reported as averages of those measured at the beginning and end of the incubation.

\section{Assumptions}

0xygen-based primary productivity was calculated from the light-and dark-bottle data. The calculations are based on the following assumptions:

1) Phytoplankton were the only source of oxygen in the sealed lightbottles.

2) Community respiration (bacteria, phytoplankton, and zooplankton) was the only oxygen sink.

3) Phytoplankton respiration was the same in the light and dark bottles. Although studies have suggested that light reduces phytoplankton respiration and increases other forms of phytoplankton oxygen consumption (Harris and Piccinin, 1977), evidence concerning the effect of light on respiration is contradictory.

4) Phytoplankton respiration is constant with depth.

5) Phytoplankton productivity per unit of light in the afternoon is the same as in the morning. Lehman and others (1975) reported that productivity per unit of light in the afternoon is lower than that in the morning. Greeson and others (1977) recommend the use of Vollenweider's method (Vollenweider, 1965) when incubations do not last for the entire dawn to dusk period. The method is based on a plot of "percent cumulative productivity versus time", with sunrise to midday accounting for 56 percent of the daily productivity and midday to sunset representing the other 44 percent for a cloudless day. Cohen and others

1/The mention of brand names in this report is for identification purposes only and does not constitute endorsement by the U.S. Geological Survey. 
(1982), Schindler and Fee (1973) and Harris and Lott (1973) have suggested that the depression of productivity in the afternoon relative to the morning is an artifact of the productivity method and is associated with inorganic carbon depletion in bottles sealed from the atmosphere. Therefore, when incubations were terminated approximately at midday due to oxygen supersaturation, primary productivity per day was calculated by assuming that productivity in the afternoon was the same as in the morning. Vollenweider (1965) suggests that reasonable day rate estimates are obtained by assuming a symmetrical daily curve of instantaneous productivity rate. Therefore, partial day incubations were expanded to day rate integrals by assuming that instantaneous productivity followed the positive portion of a sine wave.

\section{Calculations}

Primary productivity and related parameters were calculated as follows:

1) $R / H R=R_{T} / N$

2) $R_{D}=R / H R=D_{H}$

3) $G P P / D=\left(O_{A}-O_{B}\right)+R_{T}$, if incubation lasted from dawn to dusk.

4) $G P P / H=(G P P / D) / D H$

5) When incubations were terminated at mid-day, GPP/H was calculated by determining GPP for the half day incubation and dividing by the length of the daylight portion of the incubation:

5a) $G P P / H=\left(\left(O_{A}-O_{B}\right)+R_{T}\right) / 0.5 D_{H}$

5b) $\mathrm{GPP} / \mathrm{D}=\mathrm{GPP} / \mathrm{H} \cdot \mathrm{D}_{\mathrm{H}}$

6) $N P P / D=G P P / D-R_{D}$

7) $\mathrm{DBAL}=\mathrm{GPP} / \mathrm{D}-(\mathrm{R} / \mathrm{HR} \cdot 24)$

where

$R / H R=$ dark bottle respiration, $m g 0_{2} \mathrm{~L}^{-1} \mathrm{~h}^{-1}$;

$R_{T}=$ total respiration for the entire incubation period, in $m g 0_{2} \mathrm{~L}^{-1}$;

$N=$ duration of incubation, in hours;

$D_{H}=$ hours of day light, in hours;

$R_{D}=$ respiration during daylight hours, in $m g 0_{2} \mathrm{~L}^{-1}$; 


$$
\begin{aligned}
\mathrm{GPP} / \mathrm{D}= & \text { gross productivity per day, } \mathrm{mg} \mathrm{O}_{2} \mathrm{~L}^{-1}, \\
\mathrm{O}_{\mathrm{A}}= & \text { concentration of oxygen at the end of the incubation, } \\
& \text { in mg } \mathrm{O}_{2} \mathrm{~L}^{-1} ; \\
\mathrm{O}_{\mathrm{B}}= & \text { concentration of oxygen at the beginning of the incubation, } \\
& \text { in } \mathrm{O}_{2} \mathrm{mg} \mathrm{L}^{-1} ; \\
\mathrm{GPP} / \mathrm{H}= & \text { gross productivity, in mg } \mathrm{O}_{2} \mathrm{~L}^{-1} \mathrm{~h}^{-1} ; \\
\mathrm{NPP} / \mathrm{D}= & \text { net primary productivity, in mg } \mathrm{O}_{2} \mathrm{~L}^{-1} \text { per day; } \\
\mathrm{DBAL}= & \text { net daily djssolved oxygen balance for a } 24 \text {-hour period, } \\
& \text { in mg } 0_{2} \mathrm{~L}^{-1} .
\end{aligned}
$$

\section{Alternative Techniques for the Measurement of Primary Productivity}

Experiments were performed to select the procedure to measure primary productivity that would minimize artifacts due to collection and incubation techniques. The alternative techniques that were tested are listed below.

1. One-liter B.0.D. bottles were filled with samples and compared with $300 \mathrm{~mL}$ bottles to evaluate the effects of volume on primary production. Samples were collected in the Alexandria, Va., channel.

2. Depth-integrated samples were obtained in Alexandria, Va., channel by lowering an opaque rubber hose through the water column while pumping with a plastic-impeller, centrifugal pump. Sample collection through a hose is faster than with a depth-integrating bottle, but organisms may be damaged by the pump or chemicals in the hose material.

3. Dark bottles with water from the Alexandria, Va., channel were incubated in the 100-percent sunlight section of the incubation boxes and in the 6percent sunlight section and compared. Light may leak into sealed dark bottles through pinhole flaws in the plastic coatings.

4. Point samples were collected at Marbury Point with a horizontal Niskin bottle; the samples were then placed in the incubation boxes at a light intensity equivalent to that at the depth from which the sample was removed. The results were compared to those obtained from depth-integrated samples from the same station.

5. Samples taken from Alexandria were placed in 300-mL B.0.D. bottles and were suspended in the water column at depths equivalent to the following light intensities: approximately 100-percent surface light (immersed just below the water surface); $65 ; 30 ; 16$; and 6-percent 1 ight intensity, and were compared to similar samples in the incubation boxes.

6. Samples from Alexandria were incubated from 1100 to 1500 hours; daily productivity then was calculated by the method of Vollenweider (1965). The results were compared to those from all-day (10-hour) incubations. 
7. Dye-tracer studies suggest that the fresh, tidal Potomac River, from Alexandria to Hallowing Point, mixes top to bottom in one hour or less (W. E. Webb, U.S. Geol. Survey, personal commun.). To duplicate this mixing pattern, samples from Marshall Hall were moved from one light intensity in the incubation boxes to the next, consecutive intensity such that they were mixed from 100-percent to 6-percent light in one hour and back to 100-percent in the next hour. The pattern was repeated dawn to dusk.

\section{Measurement of Downwelling Irradiation}

The extinction of light in the water column was determined by measuring sunlight on the surface with the air-calibrated setting of a quantum sensor and then measuring the depth of 1 -percent light with a water calibrated setting. The depth of 1-percent light is considered the limit of the photic zone, the zone at which productivity exceeds respiration (Parsons and others, 1977).

The extinction coefficient, $k$, was determined from the Beer-LambertBouger law:

$$
I=I_{0} e^{-k} I^{z}
$$

where $\quad z=$ depth, in meters

$Z=$ depth, in meters
$I=1$ ght at depth $z$, in ueinsteins $\mathrm{m}^{-2} \mathrm{sec}^{-1}$
$\mathrm{I}_{0}=$ surface light, in ueinsteins $\mathrm{m}^{-2} \mathrm{sec}^{-1}$
$\mathrm{k}_{\mathrm{I}}=$ extinction coefficient, $\mathrm{m}^{-1}$

A Secchi depth was determined in conjunction with every extinction coefficient measurement. Secchi depth is the depth at which brightness due to scattering of light is the same as the brightness of light reflected from a Secchi disc.

Secchi depth does not yield an accurate extinction coefficient because scattering of light is related in a complex, non-linear manner to the number and size of suspended particles and to the depth at which light penetrates through the water column. The Secchi depth, however, can be a valuable source of information concerning turbidity and can be calibrated against measurements taken with a light meter. When light measurements were not available, the extinction coefficients were calculated as follows:

$$
k_{I}=\frac{1.7}{D_{s}}
$$

where $D_{S}=$ Secchi depth in meters (Idso and Gilbert, 1974). The extinction coefficient calculated using the Secchi depth tended to be $0.6 \mathrm{~m}^{-1}$ higher than that determined using the quantum light sensor (fig. 4). The coefficients determined from Secchi depth are marked with an asterisk in table 1. When more than one depth of 1-percent light or secchi depth was deter- 


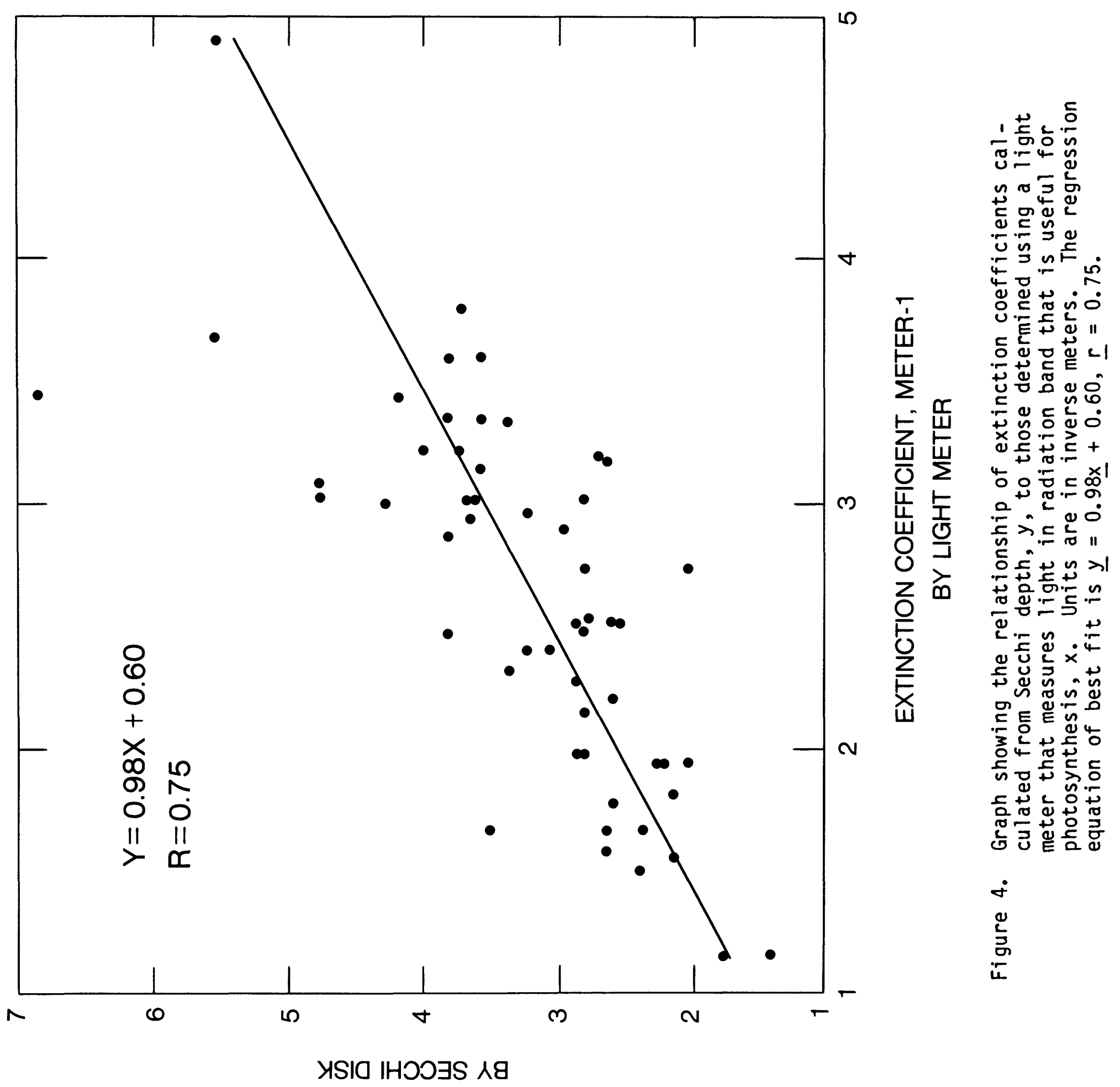

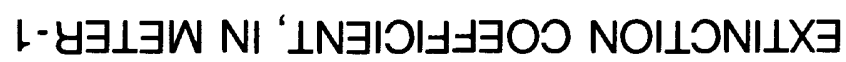


Table 1.--Solar radiation extinction coefficients, $(k)$, in the water column.

At Alexandria, Virginia (AT VA); Alexandria, Maryland channel (Al MD); Hatton Point (Hatton); Hallowing Point (Hal); Mount Vernon (MtV); Quantico (Q); and Douglas Point (Dg) stations.

Extinction coefficients were determined using quantum sensor measurements of the depth of 1 percent light except where marked: $\quad *=k$ determined by secchi disc; $t=k$ determined by quantum sensor measurements of depth of 50 percent light.

\begin{tabular}{|c|c|c|c|c|c|c|c|}
\hline \multirow[b]{2}{*}{$\begin{array}{l}\text { Productivity } \\
\text { Dates }\end{array}$} & \multicolumn{4}{|c|}{ Extinction Coefficients at, $k$, Stations } & \multirow[b]{2}{*}{ MtV } & \multirow[b]{2}{*}{$\mathrm{Dg}$} & \multirow[b]{2}{*}{$Q$} \\
\hline & A1 VA & Al MD & Hatton & $\mathrm{Hal}$ & & & \\
\hline \multicolumn{8}{|l|}{1980} \\
\hline $\begin{array}{r}05 / 22 \\
05 / 29 \\
06 / 23-06 / 24 \\
05 / 25-06 / 26 \\
07 / 23-07 / 24 \\
07 / 29-07 / 30 \\
08 / 04-08 / 05 \\
08 / 07-08 / 08 \\
08 / 13-08 / 14 \\
08 / 20-08 / 21 \\
08 / 25-08 / 26 \\
09 / 03-09 / 04 \\
09 / 15-09 / 16 \\
11 / 18-11 / 19 \\
12 / 16-12 / 17\end{array}$ & $\begin{array}{l}-2.52 \\
--.- \\
-2.52 \\
--.- \\
-2.74 \\
-2.53 \\
-2.33 \\
-1.99 \\
-3.34 \\
-3.20 \\
-3.22 \\
-2.43^{\star} \\
-2.94^{\star} \\
-2.58^{\star} \\
-1.82\end{array}$ & $\begin{array}{l}-2.33^{\star} \\
---. \\
-3.04 * \\
---. \\
-3.21 \star \\
-2.53 \\
-3.15 \\
-2.48 \\
-2.41 \\
-3.36 \\
-1.68 \\
-2.35 \star \\
-1.99 \\
---- \\
-1.51\end{array}$ & $\begin{array}{l}--. . \\
--.- \\
-\ldots- \\
--.- \\
-2.02 \star \\
-2.83 \star \\
-2.21 \\
-1.95 \\
-2.41 \\
-1.59 \\
-1.68 \\
-1.68 \\
-2.52 \\
-1.57 \\
-2.28\end{array}$ & $\begin{array}{l}-\ldots . \\
-\ldots .- \\
-\ldots- \\
--.- \\
-3.66^{\star} \\
-5.31^{\star} \\
-3.36 \\
-3.03 \\
-3.61 \\
-3.01 \\
-3.52^{\star} \\
-3.02 \\
-3.36 \\
-2.36^{\star} \\
-2.95\end{array}$ & 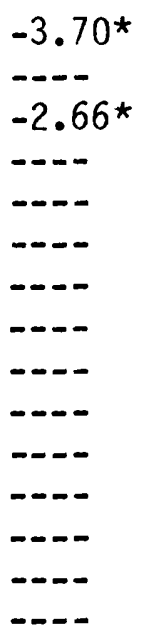 & 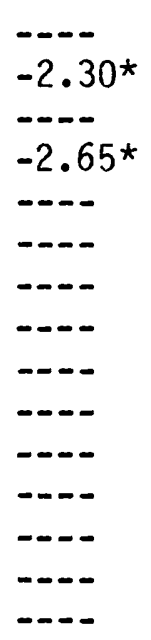 & 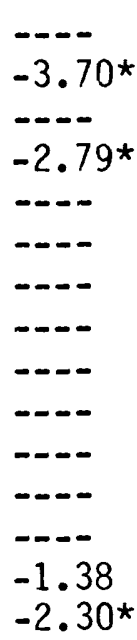 \\
\hline \multicolumn{8}{|l|}{1981} \\
\hline $\begin{array}{l}02 / 04-02 / 05 \\
04 / 01-04 / 02 \\
04 / 15-04 / 16 \\
05 / 19-05 / 20 \\
06 / 30-07 / 01 \\
07 / 08-07 / 09 \\
07 / 20-07 / 21 \\
08 / 03-08 / 04 \\
08 / 19-08 / 20 \\
08 / 25-08 / 26\end{array}$ & $\begin{array}{l}-1.16 \\
-2.58^{\star} \\
-3.68 \\
-2.52 \\
-3.03 \\
-2.43^{\star} \\
-1.33^{t} \\
-3.52 \\
-2.79^{\star} \\
-2.90\end{array}$ & $\begin{array}{l}-1.16 \\
-3.04 * \\
-3.44^{\star} \\
-2.52 \\
-2.74 \\
-2.16 \\
-3.03 \\
--- \\
-2.78 * \\
----\end{array}$ & $\begin{array}{l}-1.18 * \\
-4.72^{\star} \\
-3.09 \\
-2.48 \\
-2.88 \\
-1.78 \\
-1.95 \\
---- \\
-1.95 \\
----\end{array}$ & $\begin{array}{l}-2.54 \star \\
-6.78 \star \\
-3.44 \\
-3.60 \\
-3.22 \\
-2.97 \\
-3.62 \star \\
--- \\
-2.98 \star \\
----\end{array}$ & 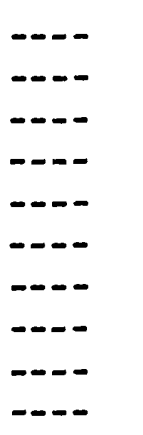 & 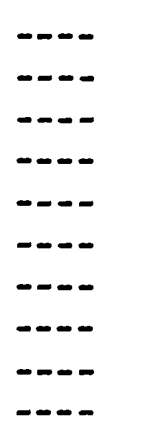 & $\begin{array}{l}-1.16 \\
-3.54 * \\
--- \\
-4.90 \\
-3.81 \\
-4.15^{\star} \\
-3.21 \\
--. \\
--- \\
---\end{array}$ \\
\hline
\end{tabular}


mined during a single day, those determined closest to the time of sample collection were averaged. All Secchi depths used were reported in Blanchard and others (1982).

The extinction coefficients permit the calculation of a depth in the water column equivalent to a percentage of surface light. The depth of 60 percent of surface light is 0.17 meter if $k_{I}=3.0$. Thus, the percentage of surface light in the productivity boxes can be converted to an equivalent depth.

\section{Calculation of Depth-integrated Productivity}

Gross productivity under a unit surface area of a water body can be calculated by integrating productivity from the surface to the bottom of the water column

$$
\int_{0}^{z} P d z
$$

where

$$
\begin{aligned}
z= & \text { depth, in meters } \\
\mathrm{P}= & \text { gross primary productivity, in units of oxygen } \\
& \text { evolved or carbon produced per unit volume per } \\
& \text { unit time. }
\end{aligned}
$$

The data required for the calculation are the gross productivities at several depths and the depth of the representative water column. The depths used were 2.2, 2.8, 3.9, and 5.6 meters at the Alexandria, Va., Maryland Channel, Hatton Point, and Hallowing Point Stations, respectively.

The percentage of surface light in the productivity boxes was converted to an equivalent depth with the extinction coefficient. The depth of one percent light is considered the compensation depth, the depth at which gross productivity equals respiration (Parsons and others, 1977). Respiration is assumed to be constant with depth. A graph of productivity and respiration with depth is shown in figure 5. In figure 5, GPP is the area under the gross productivity curve, $R$ is the area under respiration curve, and GPP minus $R$ is net productivity per square meter.

There are several ways to calculate the area under the gross productivity curve. The curve can be integrated numerically, measured on graph paper or approximated by empirical equations that are reported in the literature (Talling, 1957).

The same three data sets were analyzed by the three different techniques. First, a function that describes the productivity-depth relationship was obtained using a third degree polynomial. The areas were calculated by integrating the polynomial by Gaussian quadrature. 


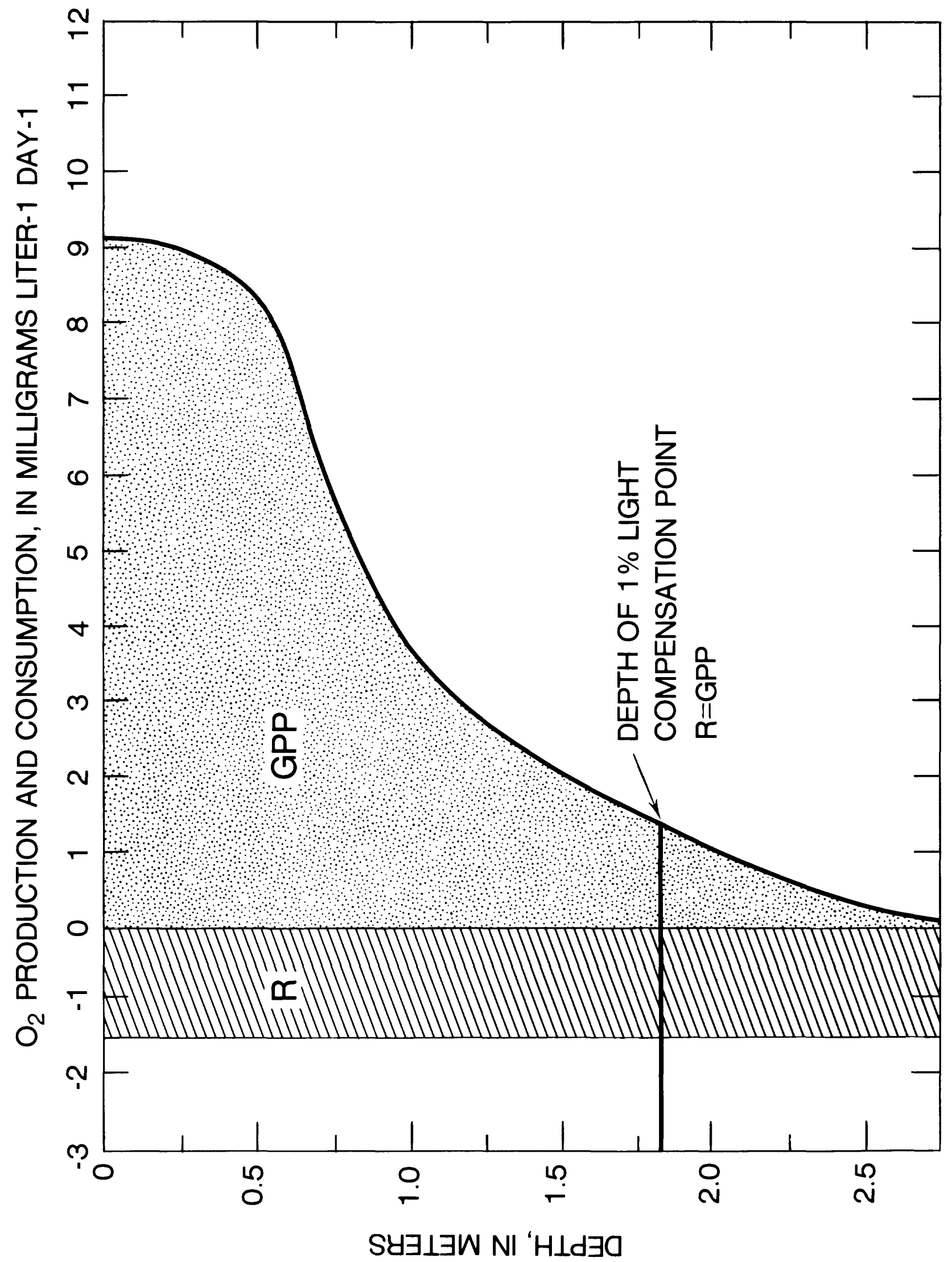

Figure 5.--Examples of gross primary productivity, GPP, and respiration, $R$, as a function of depth. 
Second, two equations were tested. One equation was derived by Talling (1957). The other was Talling's equation modified by the authors of this paper. Talling's method uses the equation

$$
\int_{0}^{H} P d z=\frac{P_{\max }}{1.33 \mathrm{k}_{I}}\left(\ln \frac{I_{0}}{0.5} I_{k}\right)
$$

where

$$
\begin{aligned}
& \mathrm{P} \quad=\text { productivity, } \mathrm{g}_{2} \mathrm{~m}^{-3} \text { day }^{-1} \\
& z=\text { depth, } m \\
& P_{\max }=\text { maximum rate of photosynthesis in the water column } \\
& k_{I}=\text { water column extinction coefficient, } \mathrm{m}^{-1} \\
& I_{0}=\text { surface light intensity, } \mu \text { einsteins } \mathrm{m}^{-2} \mathrm{sec}^{-1} \\
& I_{k}=\text { value of light intensity at which the tangent to the linear } \\
& \text { part of } P \text { versus I curve reaches a line drawn at } P_{\max } \text { (Figure 6). } \\
& H=\text { the limit of integration }
\end{aligned}
$$

The parameter $P_{\max }$ for equation (11) must be determined using the equation that describes the relationship of productivity to light intensity

$$
P=P_{\max } \frac{I}{I+K_{m}}
$$

where

$$
\begin{aligned}
& I=1 \text { ight intensity, ueinsteins } m^{-2} \text { per day } \\
& K_{m}=1 \text { ight intensity at which } P=0.5 P_{\max } \cdot
\end{aligned}
$$

A nonlinear least-squares parameter-estimation technique (Bard, 1974) was used to fit the productivity-light equation to each productivity experiiment and to estimate $P_{\max }$. In addition to $P_{\max }$, the half-saturation constant, $K_{m}$, is also obtained. Figure 6 shows the typical relationship of $P$ to $I$. Table 2 lists values of $P_{\max }$ and $K_{m}$ determined for each station and date on which a productivity measurement was made. Talling's parameter, $I_{k}$, is determined by drawing a line tangent to the initial, linear part of the $P$ versus I curve and observing where the line intercepts $P_{\max }$ (fig. 6'). We calculated depth-integrated primary productivity using Talling's equation.

Talling's parameter, $I_{k}$, is difficult to determine accurately because the slope of the initial part of the $P$ versus I graph is determined by visual inspection (Talling, 1957). The slope, however, can be calculated as the ratio of $P_{\max }$ to $K_{m}$ in which case $I_{k}=K_{m}$. The parameters $K_{m}$ and $P_{\max }$ can be determined from equation (12). The values of $I_{k}$ obtained by graphical solution were compared $k_{m}$ calculated by equation (12) for ten sets of data which yield a value of $K_{m} / I_{k}=0.59$ (Standard Deviation = $0.12)$. 


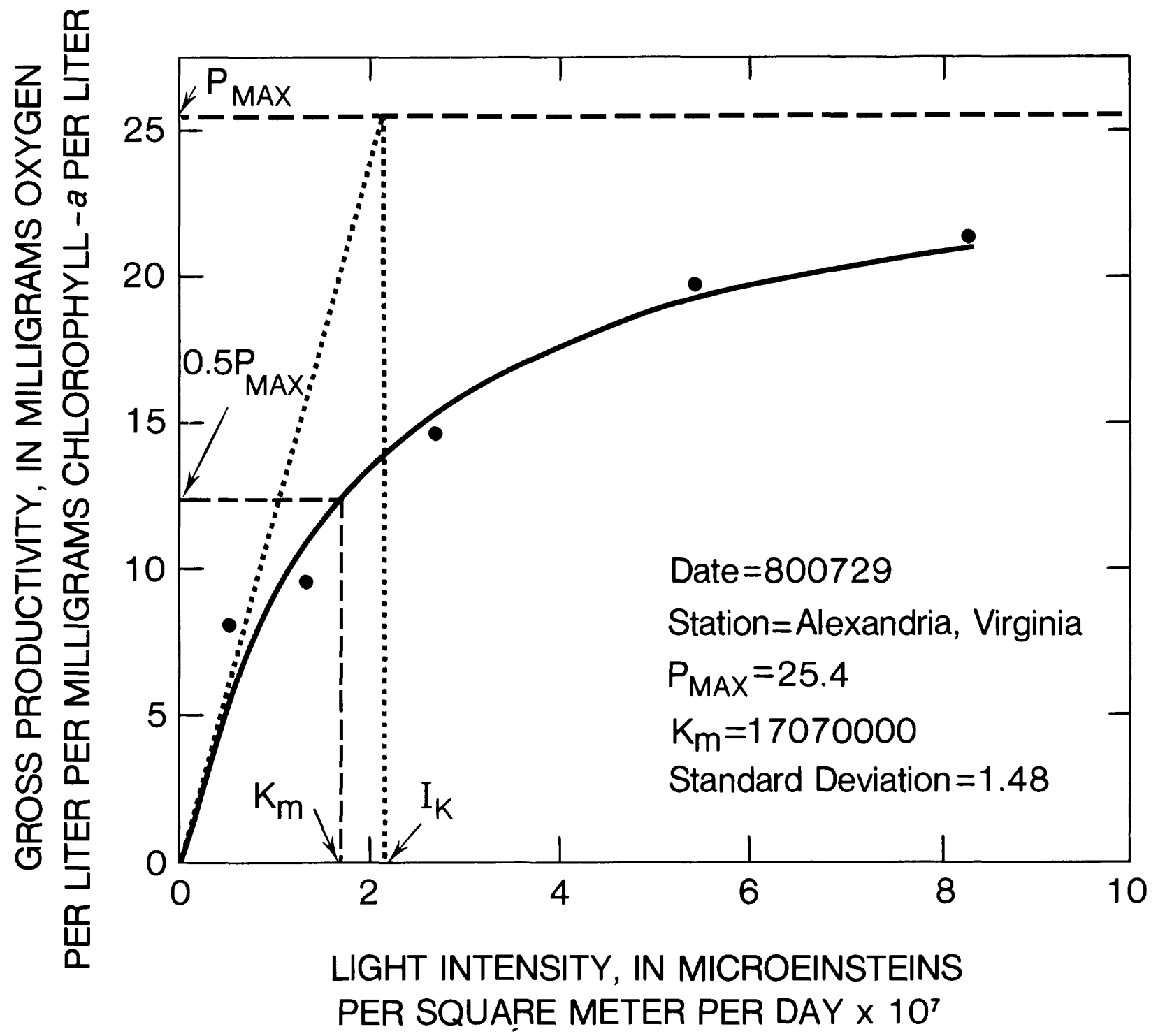

Figure 6.--Graph of gross primary productivity, in milligrams of oxygen per liter per day, as a function of light intensity, in microeinsteins per square meter per second. $\mathbb{P}_{\max }=$ maximum productivity, $K_{m}=$ half-saturation constant, $\mathrm{I}_{\mathrm{K}}=$ Talling's parameter. Solid circles are measured data; sofid line is the best non-linear, least-squares fit to the data. Horizontal, dashed line is level of $P_{\max }{ }^{-\cdots--.}$. represents line used to determine Talling's $\left.I_{k} \cdot\right]$ 


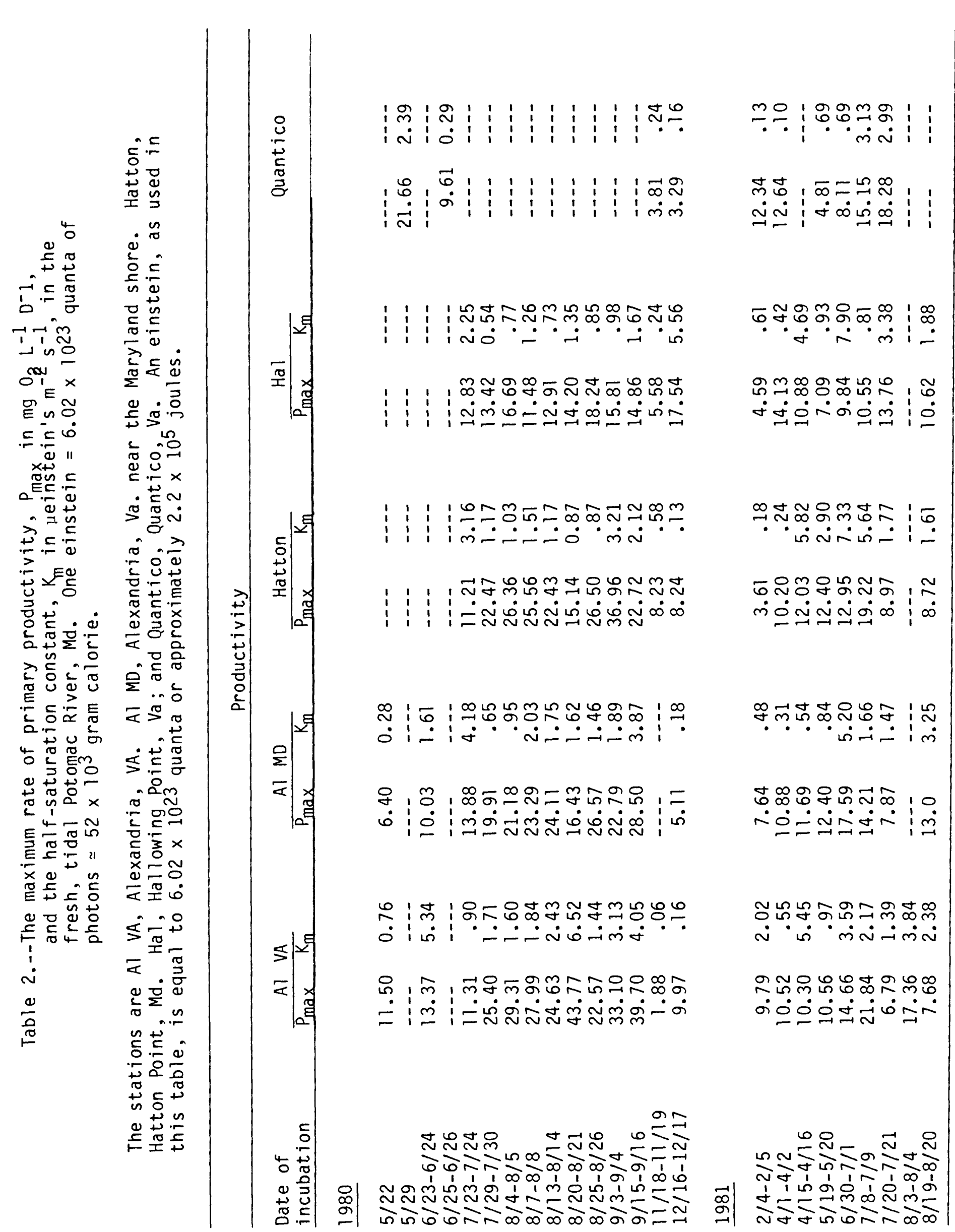


Talling used the coefficient 1.33 in equation (11) to approximate phytosynthetically active radiation (PAR) from measurements made with a thermopile (Talling, 1957). We measured PAR and did not need the coefficient 1.33. Therefore, the equation for depth-integrated primary productivity becomes

$$
\int_{0}^{H} P d z=\frac{P_{\max }}{k_{I}}\left(\ln \frac{I_{0}}{0.85 K_{m}}\right)
$$

The results obtained from equation 13 were compared to those calculated from Talling's equation.

Gross productivity data also were plotted as a function of depth on linear axes. A curve was drawn through the data points so that the area under the curve could be measured. Area was estimated by counting grid blocks. The results of the graphical determinations were used as a standard to judge the Talling and modified Talling integration methods.

\section{RESULTS AND DISCUSSION}

\section{Experimental design}

The hypothes is that "gross primary productivity and respiration determined using $300-\mathrm{mL}$ B.O.D. bottles was the same as those in 1-liter bottles" was accepted at the 5-percent significance level (table 4). Primary productivity in depth-integrated samples pumped through a hose was the same as productivity in samples collected with a polyethelene bottle (5-percent significance) (table 3). Respiration in samples taken by pump and hose, however, was significantly lower than that in samples collected with the depth-integrating bottle (5-percent significance) (table 3). The reduction in respiration due to pumping has been observed in B.0.D. experiments (W. $E$. Webb, personal commun., 1981). Respiration measured in dark bottles that were exposed to full sunlight was less than respiration in bottles shielded from full sunlight (5-percent significance) (table 3 ). The results suggest that black plastic coatings and black paint may pass light through small holes or flaws in the coatings.

Productivities determined from depth-integrated samples that were incubated at 16-percent and 6-percent light were the same as productivities in point samples collected at 1.5 meter (16-percent light) and 2.1 meter (6percent light) and incubated in 16-and 6-percent light (5-percent significance) (table 4). Depth-integrated samples yielded higher productivity measurements than those in point samples taken from and incubated at light intensities equivalent to the surface and at 0.6 meter and 0.9 meter. Bot-

tles incubated in the water column were not significantly different from those in the incubation boxes at the 100-, 65-, and 16-percent light intensities, but were significantly different at 32-percent light (5-percent significance level) (table 3 ). 
$=$
$=$
0
0
0
0
0
3
0
0
$=$
$=$

ธิ ธ่

a

危

iิ .

귱

ชั

象.

产들

ธิ

뭉

त)

0

望.

c

ᄃ

뒁

핟

돈

둥

$\stackrel{\square}{\square}$

ه

훙하

$0 \cdot \frac{1}{3}$

a o

을

통

$4 \%$

4ั 8

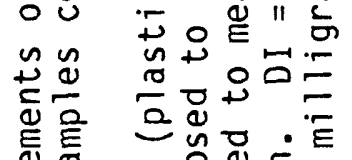

就

ড

柼

$\stackrel{\overbrace{}}{\mathbb{E}}+$

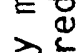

$\$$ 员

둥

옹

خัญ

응

i.

$\frac{0}{0}$ $\ddot{\sim}$

$\stackrel{\sim}{\dddot{\sigma}}$

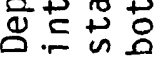

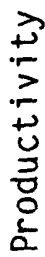

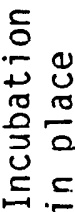

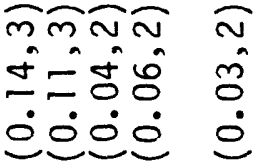

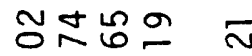

$\dot{+} \dot{\sim} \dot{-} \dot{0}$

ñ
กे
$\dot{e}$

ڤ

0

$\begin{array}{ll}\tilde{m} & \tilde{m} \\ \infty & \dot{0} \\ \dot{0} & \dot{0}\end{array}$

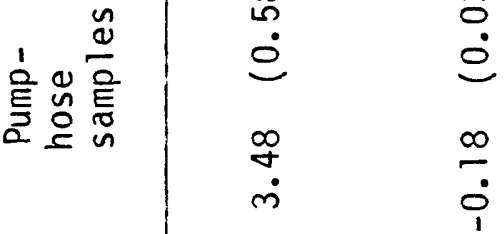

$\stackrel{5}{+}$

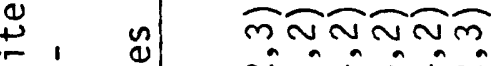

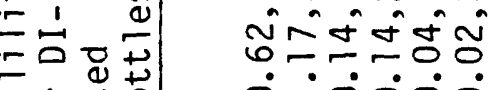

$=气 \stackrel{0}{0} \quad \dot{0}=\dot{0} \dot{0} \dot{0}$

E워

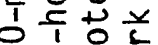

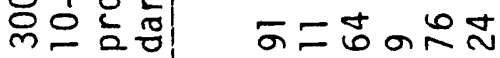

$\dot{m} \dot{\sim} \dot{0} \dot{0}$

$>\frac{4}{0}$

$\pm+$

กิ

के बे पे

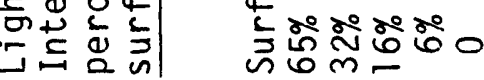




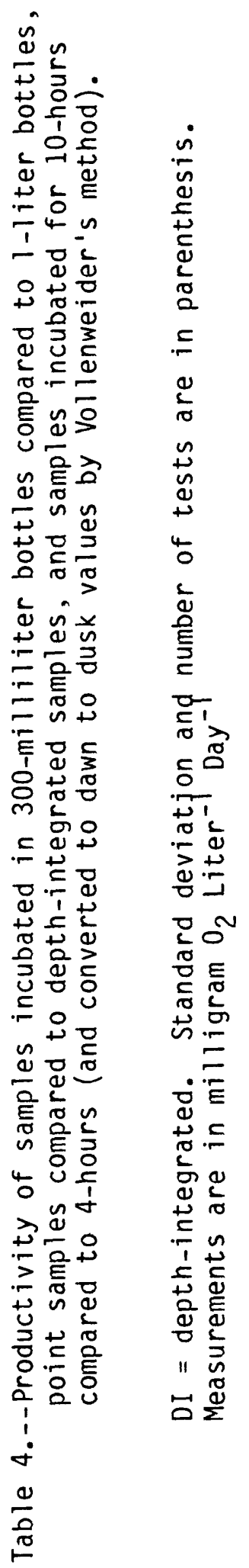

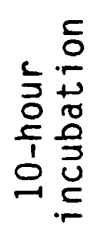

กิ กิก

ชิ

¿ $\dot{0}$

궁

ம $\dot{\nabla} \dot{~}$

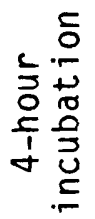

กิ กิ

$\pm \pi$

$\dot{0} \dot{0}$

ก

$\frac{3}{2}$
$\vdots$
$\stackrel{u}{z}$
0
0

○。

ติกิกติ

ใตำก

告

ิํㅇㅇํㅇํํ

லீர்

$+\frac{\mathscr{d}}{\stackrel{\circ}{\circ}}$

กิติติ

คิศึ்

0ं $\dot{0}-\dot{0}$

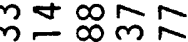

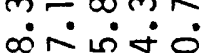

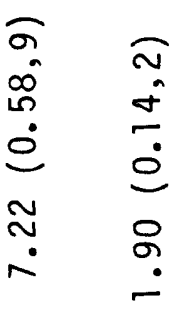

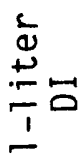

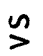

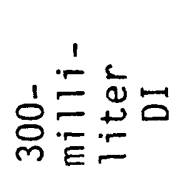

न

m

i

$=\quad 8$

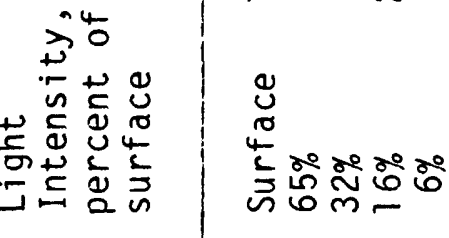


The 4 hour incubation, 1100 to 1500 hours, yielded estimates of productivity calculated by the method of Vollenweider (1965) equal to the estimates from 10-hour incubation (5-percent significance level) (table 4).

Productivity bottles that were moved from 100-percent to 6-percent 1ight and back to 100-percent light in two hours, had depth-integrated water column productivities that were higher than those found with stationary bottles (table 3).

We chose to incubate depth-integrated samples for primary productivity determinations in 300-mL B.0.D. bottles that were placed in incubation boxes and were kept at ambient temperatures. Although nylon-mesh neutral-density screens do not change the spectral distribution of light as does the extinction of light in a water column, most incubation-box determinations of productivity were not significantly different than those done in the water column. Because one liter bottles offered no advantages for productivity measurements, required greater sample volumes, and were inconveniently large, they were not used. Samples collected through an opaque hose suppressed respiration. Therefore, all samples were collected with a depth-integrating bottle. The data suggest that there may have been photosynthesis in dark bottles exposed to bright sunlight. For additional accuracy of respiration measurements, dark B.0.D. bottles were shielded from bright sunlight. The fresh, tidal Potomac River from Alexandria to Hallowing Point can be considered well mixed. Thus, point samples offered no advantage over depthintegrated samples and were more time consuming to collect. Moreover, depthintegrated samples are considered to be more representative of a well mixed system.

The procedure that was selected to measure primary productivity is shown in the flow-chart in figure 7. Samples were collected in the evening and returned to a dockside laboratory at Alexandria, Va. for processing. Light extinction was measured at each station at the time of sample collection. Dockside incubation did not require boat time necessary for water column incubations. Chlorophyll-a samples were taken from the well-mixed 20-1iter sample bottles. Samples were placed in 300-mL bottles, nutrients were added to test for nutrient limitation, dissolved oxygen was measured and the bottles were sealed and placed in the incubation boxes. The phytoplankton and suspended sediment were dispersed by rotating the bottles every hour during incubation. The incubations were terminated at dusk or at midday if degassing was obvious. Dissolved oxygen was measured in, and a chlorophyll sample was taken from each bottle. Gross and net primary productivity and respiration were calculated. The productivity-light relationship (equation (12)) was used to estimate $P_{\max }$ and $K_{m}$. Depth-integrated productivity was calculated with the modified Talling equation (13).

Productivity studies were performed monthly, from May 1980 to September 1981, several times in August, twice in September 1980, and twice during the week of August 4, 1980. The results of these efforts are presented in table 6 (in the back of the report).

The standard error of the mean for the method used for routine productivity measurements was $0.22 \mathrm{mg} \mathrm{O}_{2} \mathrm{~L}^{-1}$ day-1 for a 24 hour experiment $(n=8)$. 


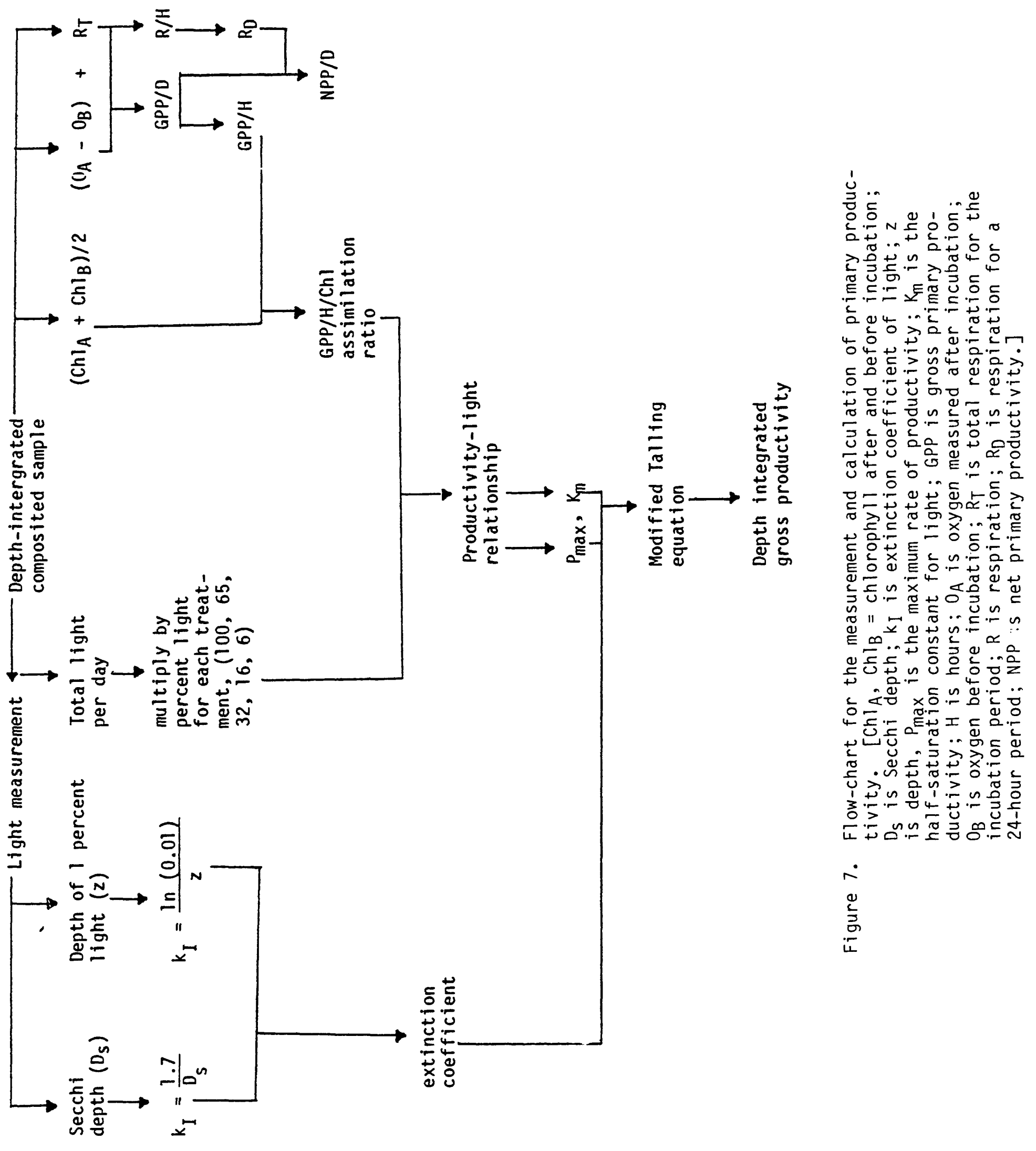


Table 5.--Depth-integrated gross primary productivity, determined by the graphical method (1), numeric integration (2),

Talling's method (3) and the modified Talling method (4).

[Measurements in milligrams $0_{2}$ per square meter per day]

\begin{tabular}{llcccc}
\hline & & & Gross Productivity & \\
\cline { 5 - 6 } Date & Station & 1 & 2 & 3 & 4 \\
\hline $7 / 29 / 80$ & Alexandria, Va. & 9.7 & 10.77 & 7.02 & 9.41 \\
$8 / 7 / 80$ & Alexandria, Va. & 18.02 & 19.11 & 14.8 & 16.84 \\
$8 / 13 / 80$ & Hatton Point & 23.62 & & 12.6 & 18.78 \\
$5 / 19 / 81$ & Hallowing Point & 1.66 & & 1.23 & 1.73 \\
$7 / 8 / 81$ & Alexandria, Va. & 8.82 & 10.94 & 6.57 & 8.92 \\
\hline
\end{tabular}


With a mean gross productivity of $6.90 \mathrm{mg} \mathrm{O}_{2} \mathrm{~L}^{-1}$ per day, there is a 95 percent probability that the population mean lies between 7.32 and $6.48 \mathrm{mg} 02$ $L^{-1}$ per day. The standard error of the mean for the oxygen probe used for all but one of the experiments was $0.022 \mathrm{mg} \mathrm{O}_{2} \mathrm{~L}^{-1}$ at a mean dissolved oxygen level of $9.93 \mathrm{mg} \mathrm{O} \mathrm{L}^{-1}(n=39)$. The standard error of the mean for chlorophyl -a measurements was $0.15 \mu \mathrm{g} \mathrm{L}^{-1}$ when the mean concentration was $62.9 \mu \mathrm{g} \mathrm{L}^{-1}(n=5)$.

\section{Depth-Integrated Productivity}

The use of the modified "Talling" equation (Talling, 1957) yielded depthintegrated productivities that were within 7 percent of the results obtained graphically for four out of five cases and was within 20 percent in one case (table 5). Talling's unmodified equation (Talling, 1957) yielded results that differed as much as 32 percent from graphically determined values.

The "modified Talling" method was used to calculate depth-integrated gross primary productivity for each station and date. The method gives results very close to those determined graphically and utilizes parameters, $P_{\max }$ and $\mathrm{K}_{\mathrm{m}}$, that are biologically meaningful. Thus, the equation can be used for predictive purposes by using experimentally determined or literature parameters. The depth-integrated productivities for each station and date are reported in table 7 . 


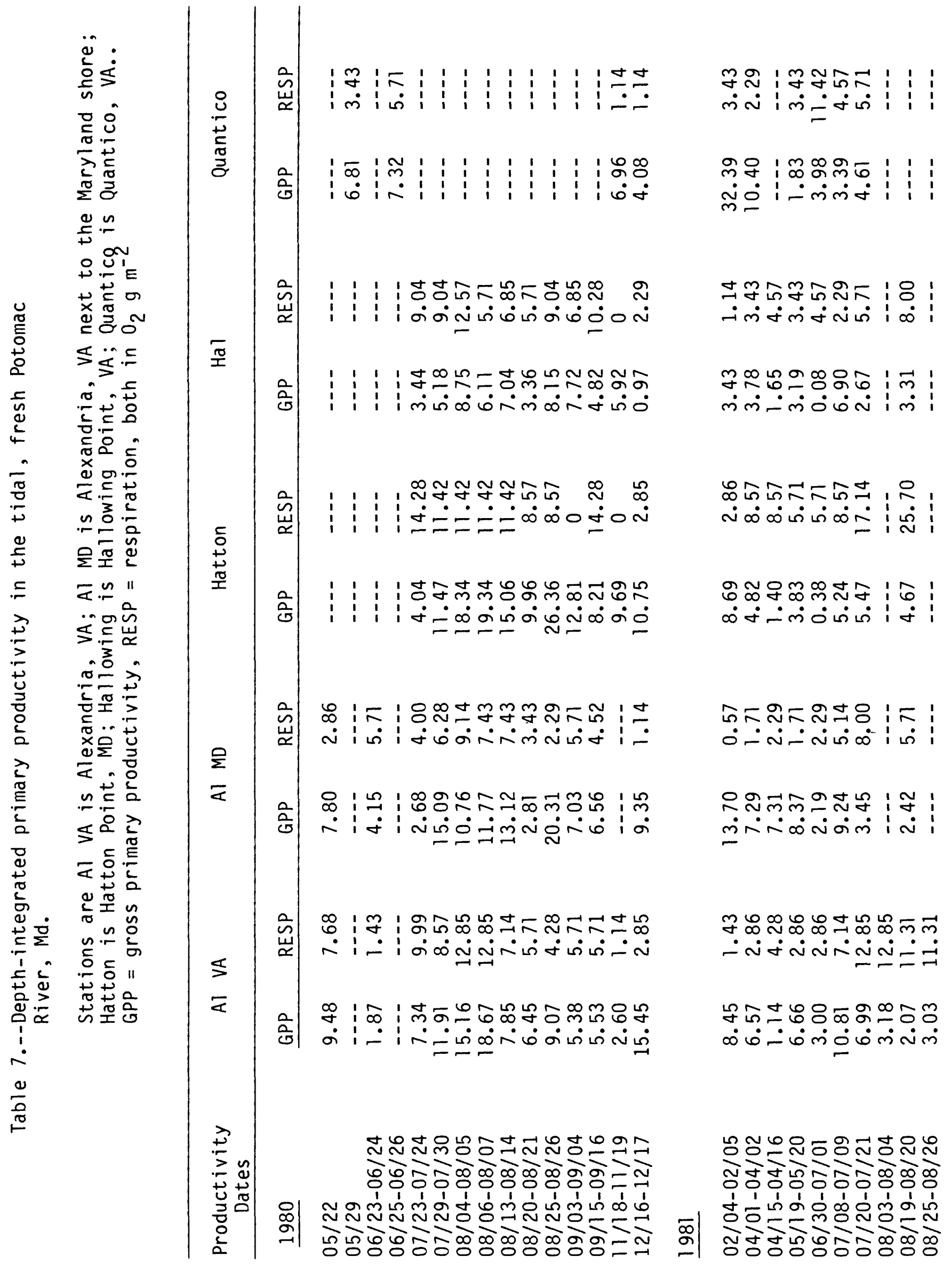




\section{References}

Bard, Y., 1974, Nonlinear parameter estimation: New York Academic Press, N.Y., 341 p.

Berman, T. and Eppley, R. W., 1974, The measurement of phytoplankton parameters in nature: Scientific Progress, 0xford v. 61, p. 219-239.

Blanchard, S. F., Coupe, R. H., Jr., and Woodward, J. C., 1982, Water quality of the Potomac River and Estuary hydrologic data report 1980 water year: U.S. Geological Survey Open-file Rept. 82-152, 330 p.

Cohen, R. R. H., Kelly, M. G., and Church, M. R., 1982, The effect of $\mathrm{CO}_{2}$ on the relationship of photosynthetic rate to ligth intensity in laboratory phytoplankton cultures: Archiv fur Hydrobiologie v. 94, no. 3, p. 326-340.

Cohen, R. R. H. Church, M. R., and Kelly, M. G., 1981 , The effect of $\mathrm{CO}_{2}$ on the response of phytoplankton productivity during a 12-hour 1ight/12hour dark light regime: Archiv fur Hydrobiologie v. 91, no. 3, p. 265-275.

Greeson, P. E., Ehlke, T. A., Irwin, G. A., Lium, B. W., and Slack, K. V., editors, 1977, Methods for collection and analysis of aquatic biological and microbiological samples: U. S. Geological Survey Techniques of Water Resources Investigations, Book 5, Chapter 4, 1977, p. 247-268.

Harris, G. P. and Lott, J. N. A., 1973, Light intensity and photosynthetic rates in Phytoplankton, Journal of the Fisheries Research Board of Canada, v. 30 , p. $1771-1778$.

Harris, G. P. and Piccinin, B. B., 1977, Photosynthesis by natural phytoplankton populations: Archiv fur Hydrobiologie, v. 80, no. 4, p. 405-457.

Idso, S. B. and Gilbert, R. G., 1974, On the universality of the Poole and Atkins Secchi disk-light extinction equation: Journal of Applied Ecology, 11 , p. 399-401.

Lehman, J. T., Botkin, D. B., and Likens, G. E., 1975, Lake eutrophication and the limiting $\mathrm{CO}_{2}$ concept: a simulation study: Verhandeln International Vereinigung Limnologie, v. 19, p. 300-307.

Marra, J., 1978, Phytoplankton photosynthetic response to vertical movements in a mixed layer: Marine Biology, v. 46, p. 203-208.

Parsons, T. R., Takahashi, M., and Hargrave, B., 1977, Biological Oceanographic Process: Pergammon Press, New York, N.Y., 2nd edition, 332 p.

Schindler, D. W. and Fee, E. J., 1973, Estimating primary production and $\mathrm{CO}_{2}$ invasion in Lake 222: Journal of the Fisheries Research Board of Canada, v. 30, p. 1501-1510. 
Stross, R. G., 1980, Growth cycles and nutrient-limited photosynthesis in phytoplankton: Limnology and Oceanography, v. 25, no. 3, p. 538-544.

Talling, J.F., 1957, Phytosynthetic characteristics of some freshwater plankton diatoms in relation to underwater radiation: New Phytologist, v. 56 , p. 29-50.

Vollenweider, R. A., 1965, Calculation models of photosynthesis depth curves and some implications regarding day rate estimates in primary productivity measurements: p. 425-457, in C. R. Goldman (ed.), Primary Productivity in Aquatic Environment. Mem. Ist. Italia Idrobiol. 18 suppl: Berkeley, Univ. of Cal. Press, $464 \mathrm{p}$. 
Table 6.--A list of the productivity and chlorophyll-a data from May 1980 to September 1981. The terms are defined in the text. Data for 100 percent light and nutrient addition experiments are an average of three tests. All other productivities are an average of two tests. NO represents the nitrate, NH the ammonia and PO the phosphate additions. Time is based on a 24 hour clock. 
ALEX MI

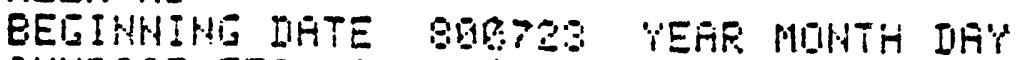

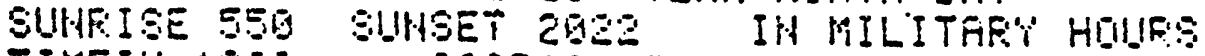

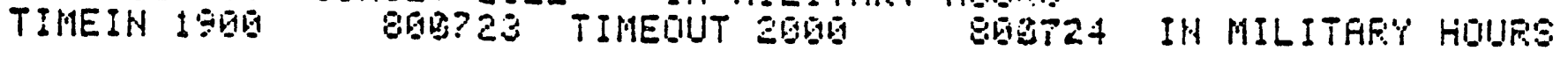

OVEFNICHT IMCUEATION

TOTFL LIGHT SUHRISE TO SUNSET 9.SE+97 MICROEIHETEIHEMMIJAY

\%LITE DR-GE R/HR EHL A GFF/H GFP/D NFF/D I ERL W/HCHL GFP/H/CHL

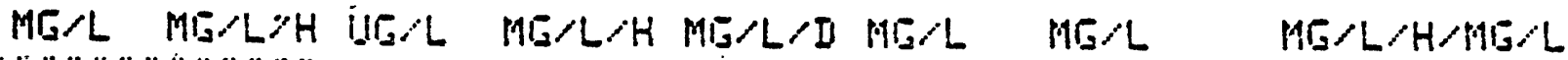

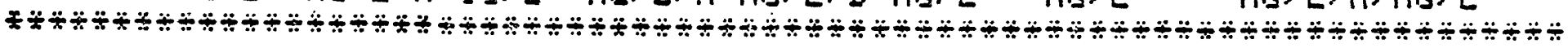

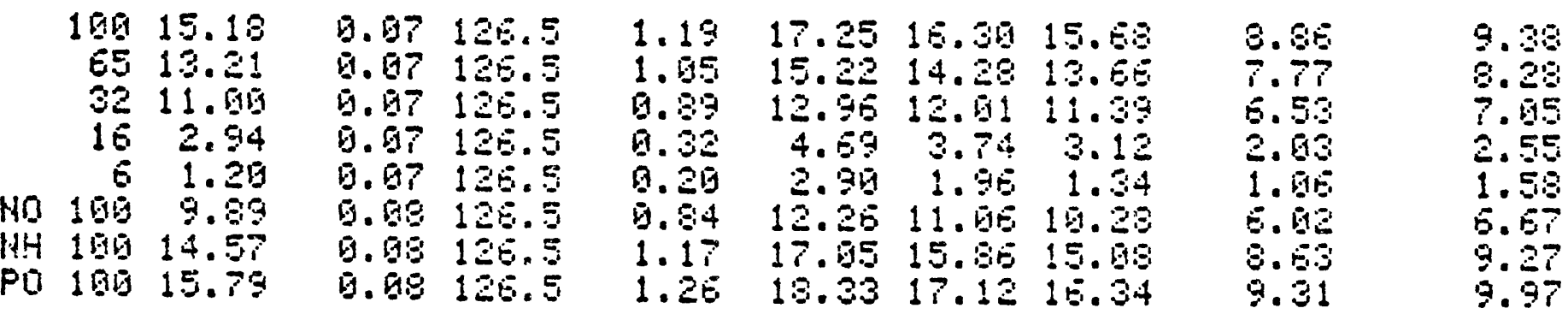

PLEY MI

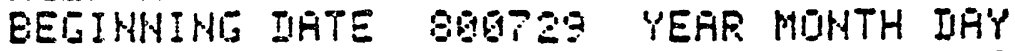

SUHFISE S5E SUHSET EQ17 IN MILITAFY HUUFS

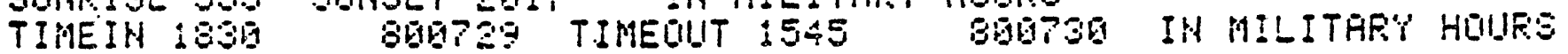

QVENHISHT INEHERTIOH

TOTHL LIGHT SUHFISE TO SUHSET 8.3E7 MILFIEIHETEIHSM MONAY

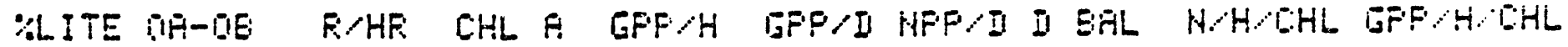

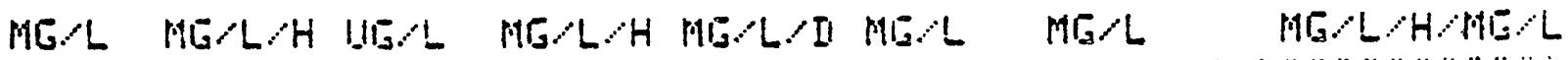

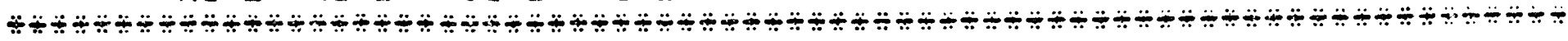

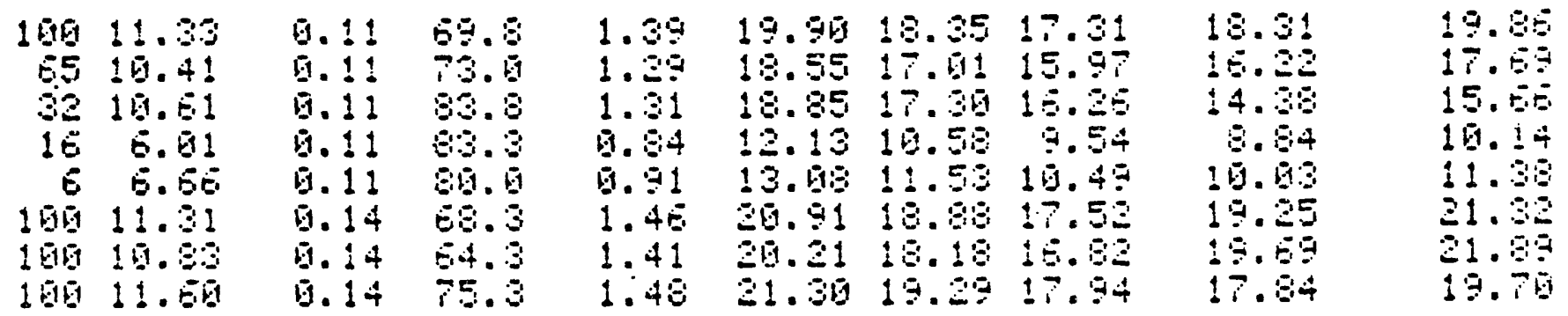


FLEX MII

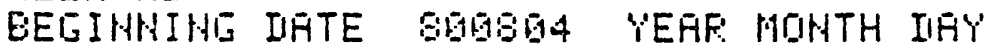

SUHFISE 559 SUHEET 2012 IN MILITHFY' HOUFS

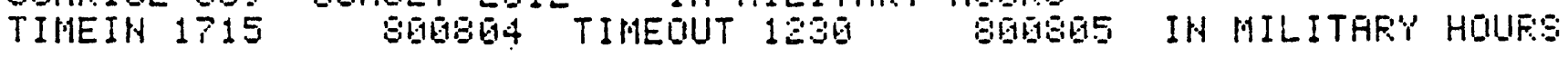

OUEFHIGHT IMLUEATIDH

TOTHL LIGHT SUHFISE TO SUHEET B.0E7 MICROEIHETEIHSM MIF'

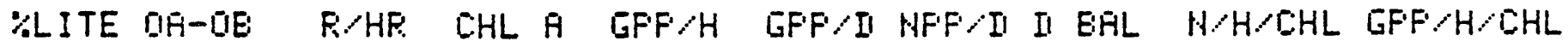

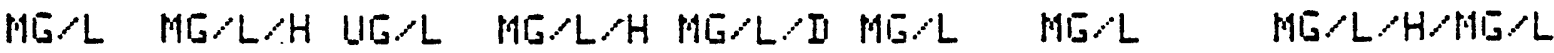

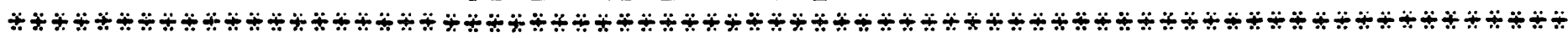

\begin{tabular}{|c|c|c|c|c|c|c|}
\hline $\begin{array}{rr}100 & 3.18 \\
65 & 6.55 \\
32 & 7.58 \\
16 & 5.17 \\
6 & 1.44 \\
180 & 9.45 \\
100 & 19.92 \\
160 & 11.93\end{array}$ & $\begin{array}{l}0.15 \\
0.15 \\
0.15 \\
0.16 \\
0.16 \\
0.15 \\
0.15 \\
0.15\end{array}$ & $\begin{array}{r}95.3 \\
105.5 \\
192.5 \\
109.3 \\
88.3 \\
101.3 \\
100.5 \\
105.3\end{array}$ & $\begin{array}{l}1.57 \\
1.77 \\
1.62 \\
1.25 \\
9.69 \\
1.99 \\
1.99 \\
2.37\end{array}$ & $\begin{array}{r}25.55 \\
25.17 \\
23.51 \\
17.30 \\
9.65 \\
29.23 \\
28.20 \\
35.65\end{array}$ & $\begin{array}{l}24.55 \\
22.55 \\
25.801 \\
15.55 \\
7.45 \\
25.05 \\
25.11 \\
31.018\end{array}$ & $\begin{array}{r}22.83 \\
21.44 \\
13.29 \\
14.97 \\
5.93 \\
24.52 \\
24.51 \\
29.36\end{array}$ \\
\hline
\end{tabular}

FLEX MD

BEGINHING DATE 390807 YERR MONTH DAY

SUNRISE 6G4 SUNSET ZQGQT IN MILITFRY HOUES

TIMEIN 1345 89930\% TIMEOUT 1245 8EG008 IN MILITAR'Y HOURE

OVERNIGHT INCUEATION

TOTRL LIGHT SUNRISE TO SUNSET 1.1EBMICROEIHSTEINS/MZ/DFY

\%LITE OA-OB R/HR CHL A GPP/H GPP/D NPP/D D ERL N/H/CHL GPP/H/CHL

MG/L MG/L/H UG/L MG/L/H MG/L/D MG/L MG/L MG/L/H/MG/L

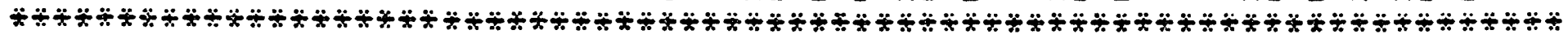

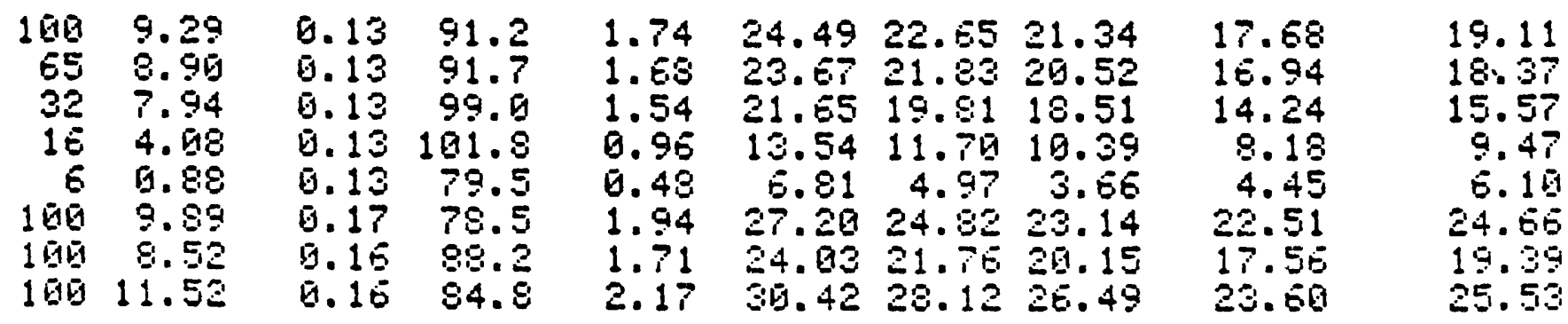


FLEX MII

BEEIHHIHE IATE BGQS13 TEAF MONTH IAY

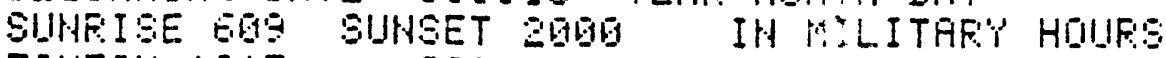

TIMEIH 1815 BGES13 TIMEOUT 1245 BGES14 IH MILITHFY HDIFS

QVERHILHT IHCIEEATIDH

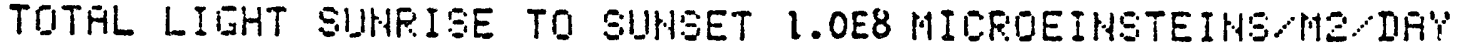

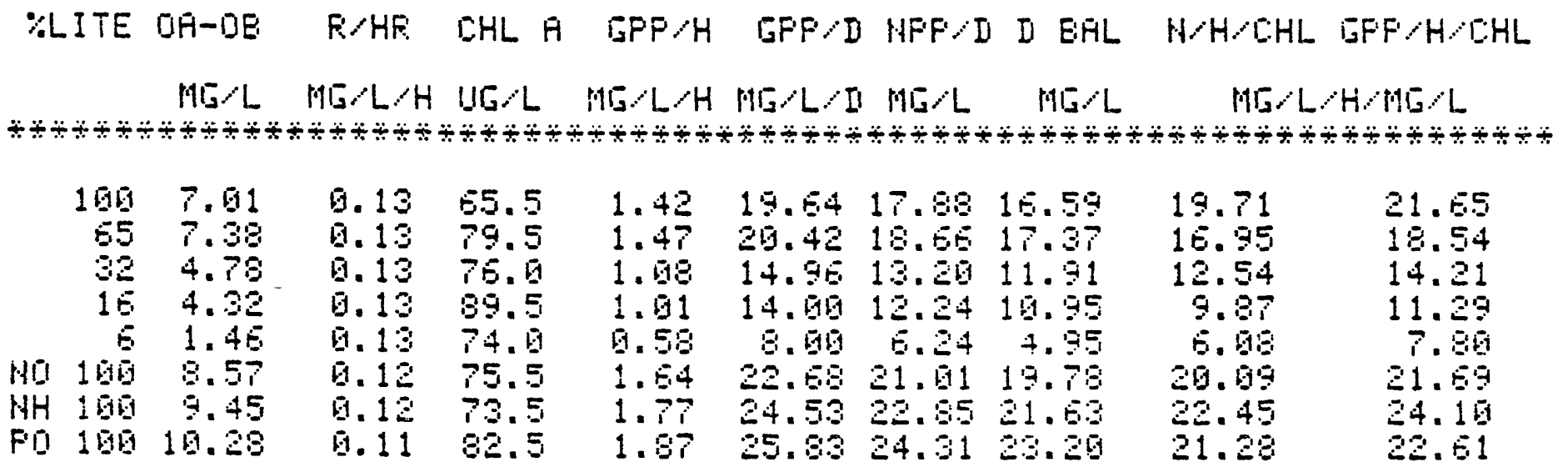

ALEX MD

EEEINNIHE DHTE 300829 YEAR MONTH DAY

SIIHRISE B14 SUNSET 1954 IN MILITARY HOURS

TIMEIN 1915 BQDE2E TIMEOUT 1315 BEEB21 IN MILITARY HOURS

QWEFNIGHT INCUBATIOH

TOTFL LIIHT SUNRISE TO SUMSET 3.5E7 MICROEINSTEINS/MZ IITY

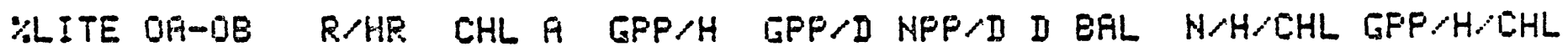

MG/L MG/L/H UG/L MG/L/H ME/L/D ME/L MG/L ME/L/H/MIJLL

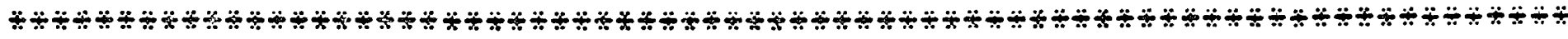

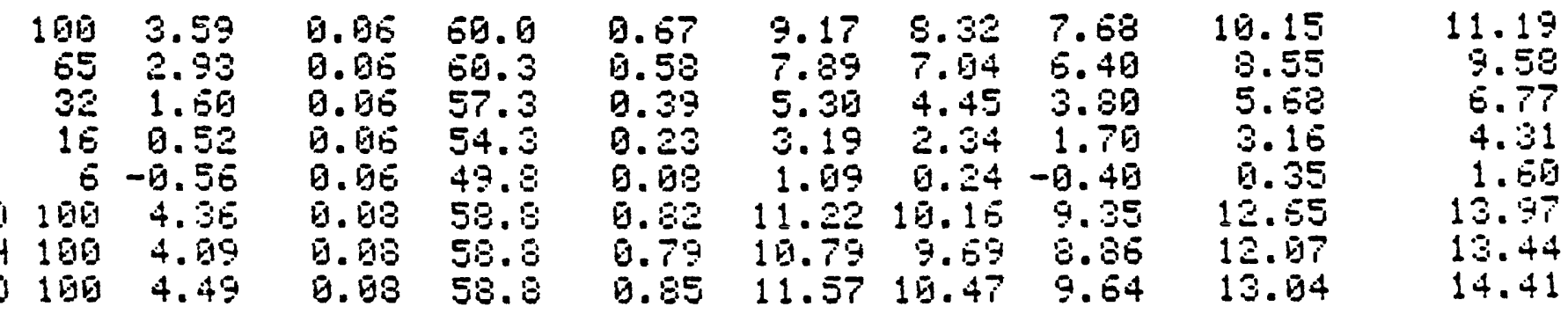


RLEX MI

BEGIHHIHS IATE BGGSQS YERR MOHTH INAY

SUHFISE EZG SUHSET 1942 IH MILITARY HOUFS

TIMEIN 21IS BEBSES TIMEOUT 130 B

OUERHISHT INIUEATION

TOTHL LIEHT BUHFISE TO SUNGET 8.1E7MICROEINGTEIHSMM,IAY

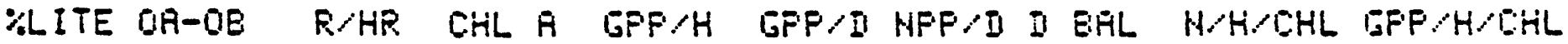

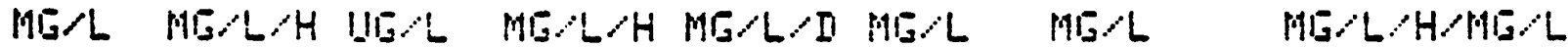

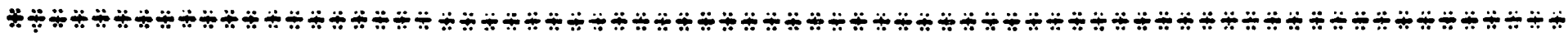

\begin{tabular}{|c|c|c|c|c|c|c|c|}
\hline $\begin{array}{rr}199 & 1.85 \\
65 & 2.45 \\
32 & 2.04 \\
16 & 1.15 \\
6 & 0.15 \\
1010 & 2.45 \\
105 & 2.54 \\
105 & 2.45\end{array}$ & 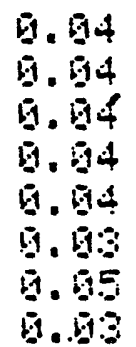 & $\begin{array}{l}18.4 \\
30.3 \\
25.6 \\
20.8 \\
19.6 \\
16.3 \\
20.0\end{array}$ & 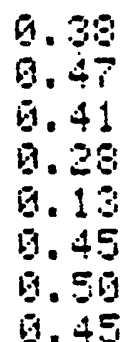 & $\begin{array}{l}5.99 \\
6.32 \\
5.47 \\
3.71 \\
1.88 \\
5.97 \\
5.72 \\
5.97\end{array}$ & $\begin{array}{l}4.51 \\
5.73 \\
4.85 \\
3.13 \\
1.15 \\
5.55 \\
5.53 \\
5.53\end{array}$ & 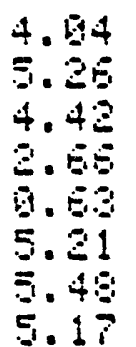 & 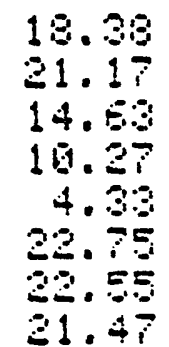 \\
\hline
\end{tabular}

RLE: MI

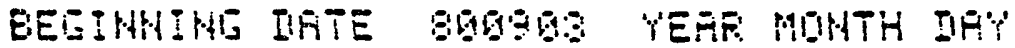

SUHFISE SQS SUHSET IOS: IH MLLITHE'T HOHFS

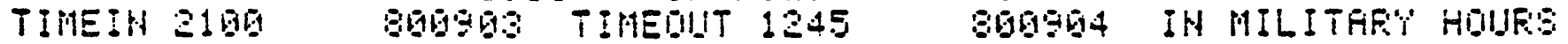

gVEFHIGHT IHCUEATIDH

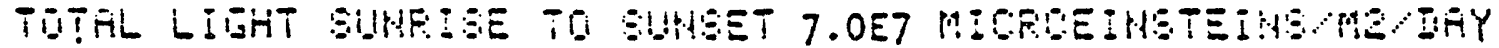

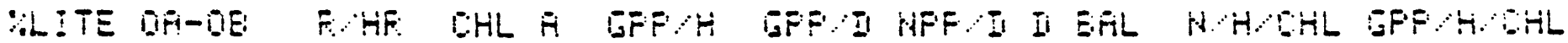

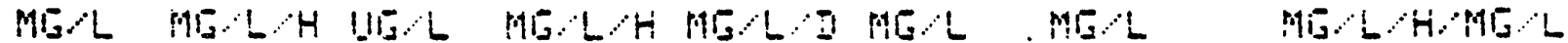

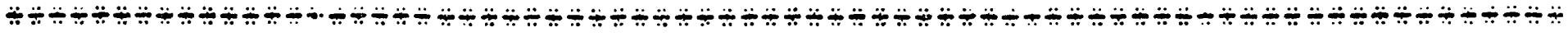

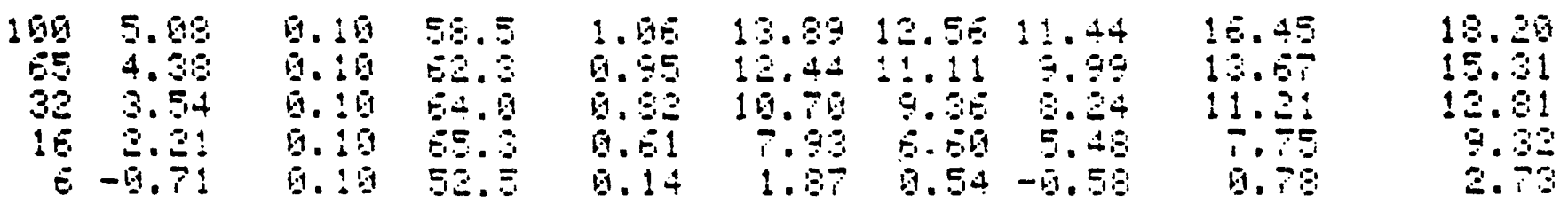


HLE: MI

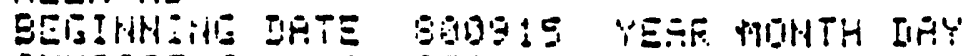

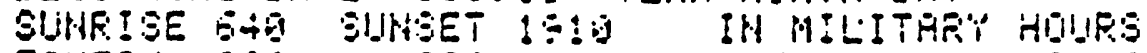

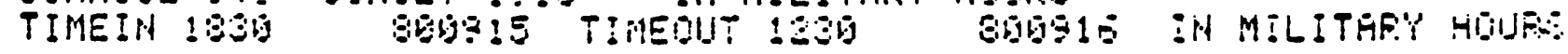

Q!EFHIEHT IMIUEETT!ÜH

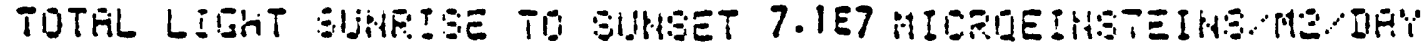

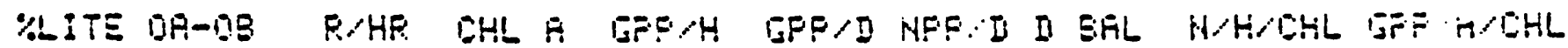

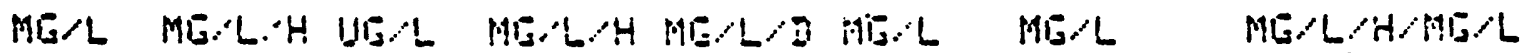
品

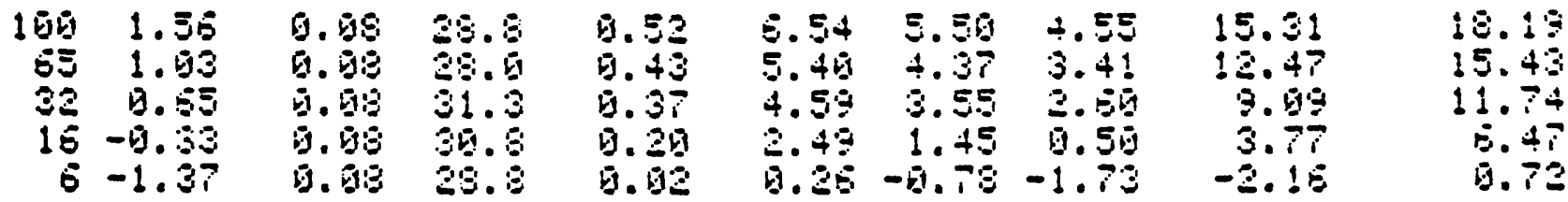

RLEX MD

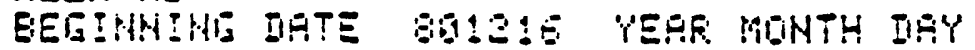

SUHFTSE TIS SHLET IBSE TH MILITART HOLRS

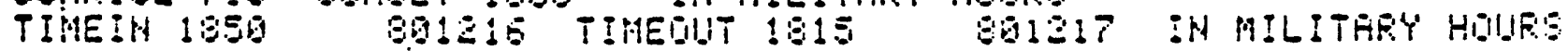

OHERHIEHT IMLHEATIOH

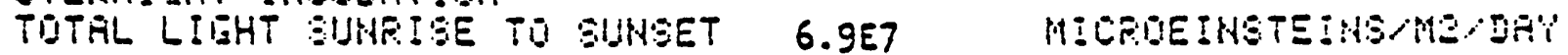

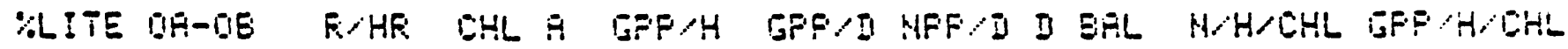

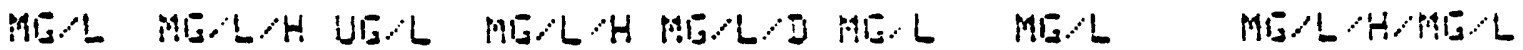
ب

\begin{tabular}{|c|c|c|c|c|c|c|c|}
\hline 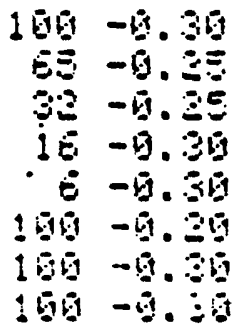 & $\begin{array}{l}9.82 \\
9.03 \\
9.92 \\
9.92 \\
9.92 \\
0.92\end{array}$ & $\begin{array}{l}3.1 \\
3.5 \\
3.3 \\
3.4 \\
3.6 \\
3.3 \\
3.5 \\
3.1\end{array}$ & $\begin{array}{l}9.91 \\
4.02 \\
9.02 \\
6.01 \\
0.01 \\
0.92 \\
0.91\end{array}$ & 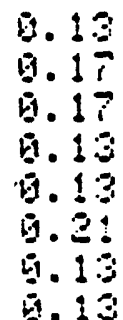 & 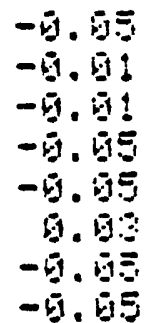 & 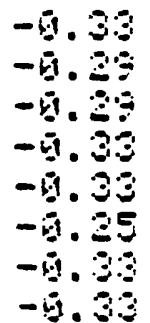 & $\begin{array}{r}-1.79 \\
-6.39 \\
-6.39 \\
-1.53 \\
-1.48 \\
1.1 ! \\
-1.95 \\
-1.81\end{array}$ \\
\hline
\end{tabular}

Fi 1500000

b.

HLEX ME:

BEGINHING IIATE 810204 YEAR MONTH DFY

SUNRISE 706 SUNSET 1722 IN MILITEFY HOURS

TIMEIN IJ45 810204 TIMEOUT I715 810205 IN MILITEEY HOURS

GIVERNIGHT INCUEATION

TOTAL LJGHT SINRISE TO EUNSET 7.7ET MICEDEIWSTEINGMAMAY

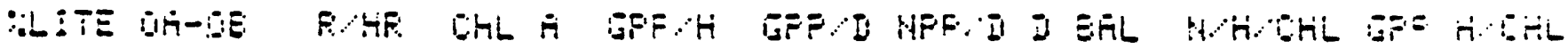

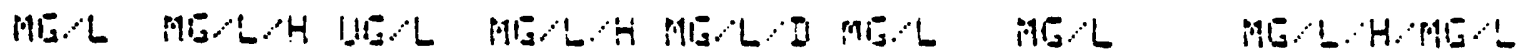

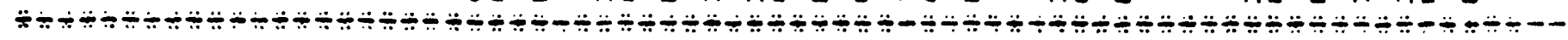

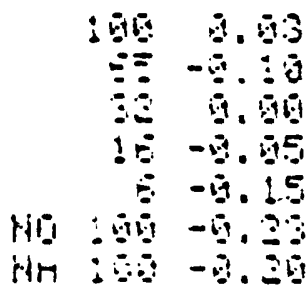

1.

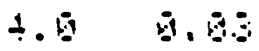

两. 34

4. 20

9.55

5.34

8.37

䭪:

$\therefore .50 .3$

B.

9. 0 - 0.60

4.8

B. 31

1. :

it. 1

+.

4.25

1.: $1-9.6$

B.

. 30

$4.7 \quad 5.5$

舟

‥ 15

百

$\therefore$.

亩.

$-8.6 \div-3$.

$P_{i j}$

6. 5 !

$\div$.

B. 1:- - $1:-6.12$

G.1

6. $80-5$

…3

4.20

3.13

ㄷ.

$-6.95$

4.93

7.15

5

6. i!

- 19 :

?.

6.35

1.83

2. 53 
ALEX MD

EELIHNTHI IHTE BIG4G: YEAF MOHTH DHY

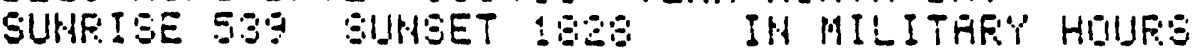

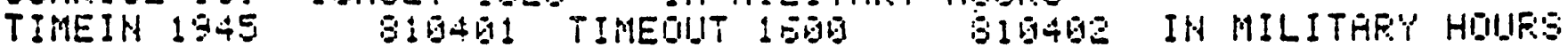

QVEFHIEHT INEUERTIDN

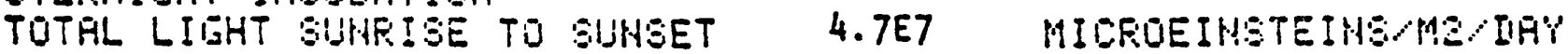

\%LITE DIA-OE R/HR CHL A GFF/H IFF/D HFF/D D ERL N/H/CHL ISFP/H/DHL

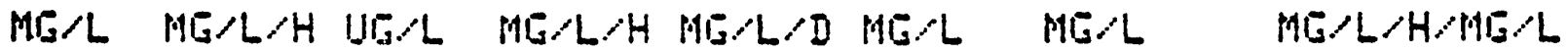

•

\begin{tabular}{|c|c|c|c|c|c|c|c|c|}
\hline $\begin{array}{l}9.32 \\
1.21 \\
0.93 \\
6.93 \\
6.20 \\
0.95 \\
1.15\end{array}$ & 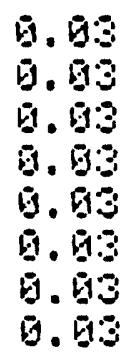 & 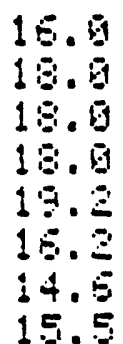 & $\begin{array}{l}5.10 \\
9.15 \\
0.15 \\
0.15 \\
0.19 \\
0.15 \\
0.15 \\
0.10\end{array}$ & $\begin{array}{l}1.99 \\
2.35 \\
2.01 \\
2.01 \\
1.10 \\
2.04 \\
2.29 \\
-69\end{array}$ & $\begin{array}{l}1.55 \\
1.95 \\
1.59 \\
1.57 \\
6.57 \\
1.51 \\
1.54 \\
1.59\end{array}$ & $\begin{array}{l}1.13 \\
1.54 \\
1.97 \\
1.19 \\
0.25 \\
1.23 \\
1.45 \\
1.21\end{array}$ & $\begin{array}{l}7.59 \\
8.31 \\
5.36 \\
5.36 \\
2.75 \\
7.74 \\
3.34\end{array}$ & $\begin{array}{r}4.35 \\
8.70 \\
3.70 \\
4.48 \\
4.84 \\
13.18 \\
10.23\end{array}$ \\
\hline
\end{tabular}

FLE. MI

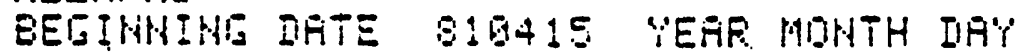

SUHFISE S2S SUHBET 1830 IN MILITAR'T HUISO

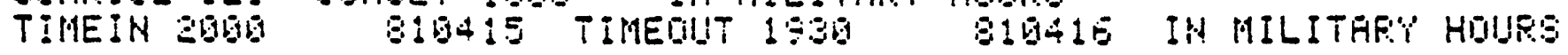

QVEFHISHT INCIEERTIDH

TOTAL LIGHT GIHATSE TO SUHSET 9.4E7 MIEFUEIHETEIHSMO IHY

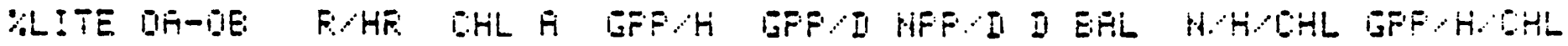

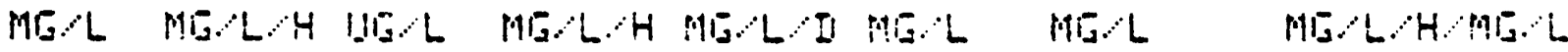
自前-

\begin{tabular}{|c|c|c|c|c|c|c|c|}
\hline 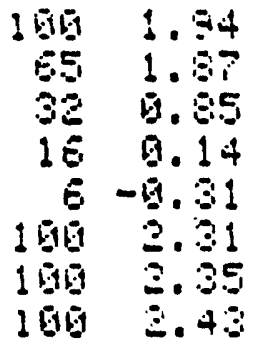 & $\begin{array}{l}5.54 \\
6.54 \\
0.54 \\
6.04 \\
0.04 \\
6.04 \\
6.04 \\
6.54\end{array}$ & 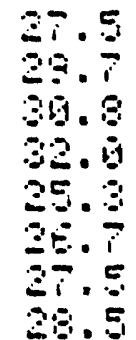 & 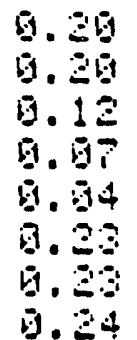 & $\begin{array}{l}2.55 \\
3.59 \\
1.53 \\
1.95 \\
6.54 \\
3.515 \\
3.44 \\
3.12\end{array}$ & 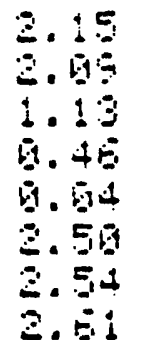 & 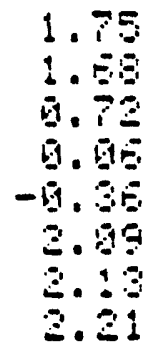 & $\begin{array}{l}5.91 \\
5.31 \\
5.73 \\
1.13 \\
9.19 \\
7.67 \\
5.97 \\
5.92\end{array}$ \\
\hline
\end{tabular}


H!EEX MII

EEEINHING DATE EIDS: YERF MOHTH IAY

SUHFISE 441 SULFET 1912 IN MILITARYY HOILIFS

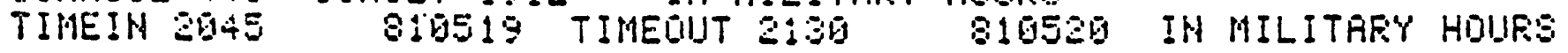

OWEFHIGHT INEUERTIOH

TOTRL LISHT SUHRISE TO SUHSET $8.0 E 7$ MICROEIHETEIHE MZ DAY

\%LITE OH-DE R/HR CHL A GFF/H GFP/I HFF/D D ERL W/H/CHL GFF/H/CHL

MG/L MG/L/H UIG/L ME/L/H ME/L/D ME/L ME/L MG/L/H/MIG/L

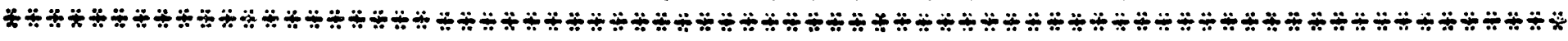

\begin{tabular}{|c|c|c|c|c|c|c|c|c|c|}
\hline $\begin{array}{r}1515 \\
65 \\
32 \\
16 \\
6 \\
100 \\
150\end{array}$ & $\begin{array}{l}1.15 \\
1.01 \\
0.75 \\
0.20 \\
0.37 \\
1.15 \\
1.35 \\
1.13\end{array}$ & 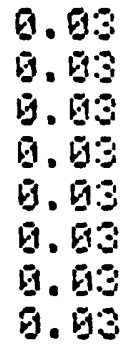 & $\begin{array}{l}8.8 \\
11.5 \\
19.7 \\
11.5 \\
19.2 \\
9.5 \\
12.0 \\
3.7\end{array}$ & $\begin{array}{l}0.12 \\
0.11 \\
0.09 \\
0.07 \\
0.07 \\
0.12 \\
0.13 \\
0.12\end{array}$ & $\begin{array}{l}1.79 \\
1.57 \\
1.35 \\
6.95 \\
1.01 \\
1.79 \\
1.84 \\
1.72\end{array}$ & $\begin{array}{l}1.23 \\
1.18 \\
0.87 \\
6.47 \\
0.54 \\
1.20 \\
1.35 \\
1.24\end{array}$ & $\begin{array}{l}0.92 \\
0.79 \\
0.55 \\
0.15 \\
0.25 \\
0.92 \\
1.95 \\
0.93\end{array}$ & $\begin{array}{l}9.53 \\
6.58 \\
5.59 \\
2.85 \\
3.54 \\
6.93 \\
7.83 \\
5.85\end{array}$ & $\begin{array}{r}3.5 \\
9.44 \\
5.55 \\
5.70 \\
6.95\end{array}$ \\
\hline
\end{tabular}

FLEX MI

EEEIHHING IITTE EIGSZB YEAF: MONTH IITY

SUHRISE 534 SUHBET $2013 \%$ IN MILITARY HOUR:S

TIMEIN ZŬG 316536 TIMEOUT 2115 S19701 IN MILITART HOURS

OUEFHIEHT IHCUEATION

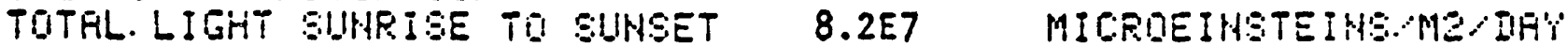

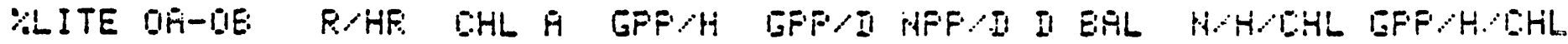

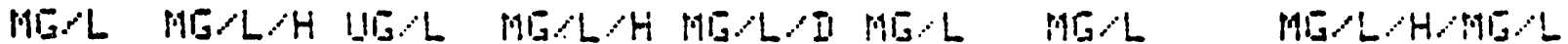

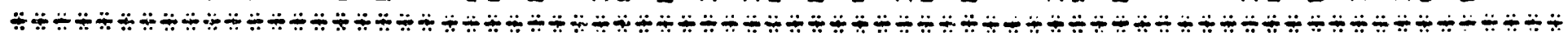

\begin{tabular}{|c|c|c|c|c|c|c|c|c|}
\hline $\begin{array}{rr}106 & 4.75 \\
65 & 3.45 \\
32 & 2.75 \\
16 & 1.18 \\
6 & -6.20 \\
190 & 4.59 \\
190 & 5.75 \\
100 & 4.81\end{array}$ & 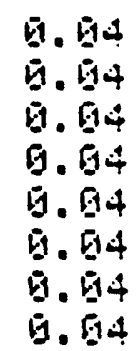 & $\begin{array}{l}20.9 \\
31.2 \\
52.4 \\
31.0 \\
35.5 \\
56.8 \\
44.5 \\
45.0\end{array}$ & $\begin{array}{l}0.37 \\
0.2 \% \\
0.24 \\
0.14 \\
0.05 \\
0.35 \\
0.43 \\
0.37\end{array}$ & $\begin{array}{l}5.51 \\
4.27 \\
3.51 \\
3.18 \\
5.78 \\
5.35 \\
6.48 \\
5.57\end{array}$ & $\begin{array}{l}4.95 \\
3.57 \\
3.61 \\
1.515 \\
9.18 \\
4.95 \\
5.87 \\
4.96\end{array}$ & $\begin{array}{r}4.54 \\
3.30 \\
3.54 \\
1.13 \\
-6.13 \\
4.39 \\
5.51 \\
4.0 .6\end{array}$ & 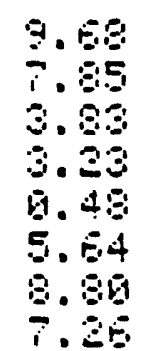 & 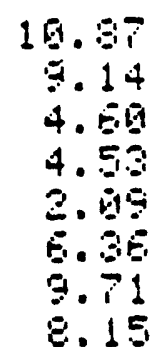 \\
\hline
\end{tabular}


FLLEY MI

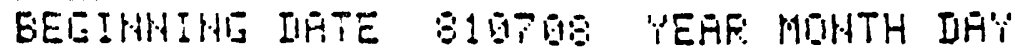

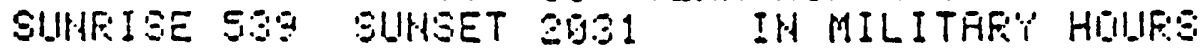

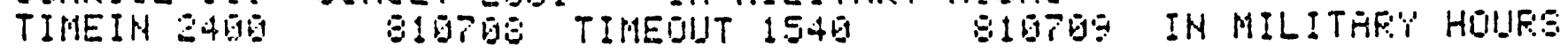

OHEEHIILHT IHCUEATTOH

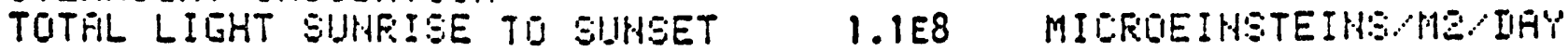

\%LITE OH-OE R/HR CHL R GFF/H GPP/I HFF/I D EHL M/H CHL GFF/H CHL

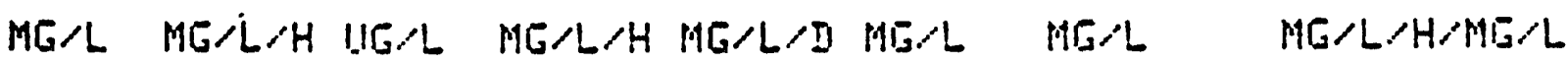

子

\begin{tabular}{|c|c|c|c|c|c|c|c|c|c|}
\hline $\begin{array}{r}160 \\
65 \\
32 \\
16 \\
6 \\
100 \\
106\end{array}$ & 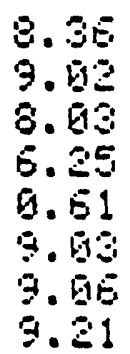 & 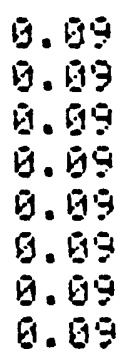 & $\begin{array}{r}75.5 \\
193.7 \\
98.6 \\
67.9 \\
7 \% .5 \\
87.3 \\
80.6 \\
9.9\end{array}$ & $\begin{array}{l}0.98 \\
1.05 \\
0.95 \\
9.75 \\
0.21 \\
1.05 \\
1.05 \\
1.07\end{array}$ & $\begin{array}{l}14.65 \\
15.58 \\
14.11 \\
11.47 \\
3.10 \\
15.60 \\
15.54 \\
15.87\end{array}$ & $\begin{array}{r}13.25 \\
14.15 \\
12.71 \\
19.67 \\
1.70 \\
14.19 \\
14.24 \\
14.46\end{array}$ & $\begin{array}{r}13.34 \\
13.32 \\
11.85 \\
0.21 \\
6.03 \\
13.38 \\
13.38 \\
13.56\end{array}$ & $\begin{array}{r}11.54 \\
4.210 \\
8.57 \\
7.79 \\
1.44 \\
14.10 \\
11.59 \\
9.74\end{array}$ & $\begin{array}{r}18.11 \\
6.58 \\
8.78 \\
9.5\end{array}$ \\
\hline
\end{tabular}

ALE: MI

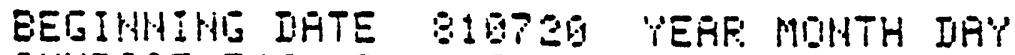

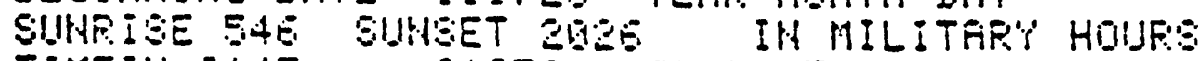

TIMEIN 2145 S19720 TIMEOUT 1445 B16721 IN MILITAEY HOURS

OVEFRIILHT INCHEATION

TOTAL LIEHT SUHFISE TO SUHSET 8.6ET MICRIEIHSTEIHSMEIIAY

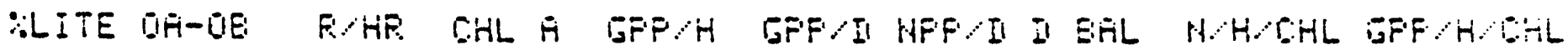

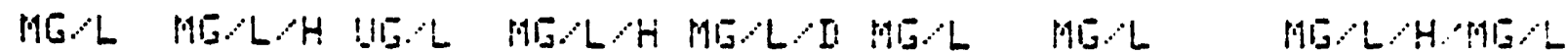

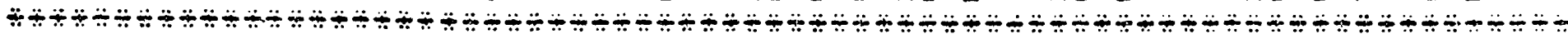

\begin{tabular}{|c|c|c|c|c|c|c|c|}
\hline $\begin{array}{r}196 \\
50 \\
30 \\
10 \\
100 \\
196 \\
196\end{array}$ & $\begin{array}{l}5.27 \\
4.95 \\
3.55 \\
2.35 \\
9.25 \\
5.71 \\
6.12 \\
6.85\end{array}$ & 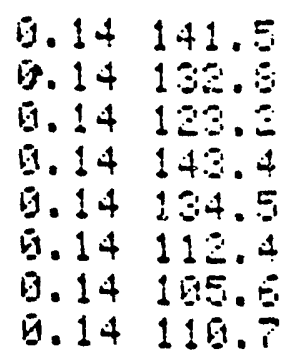 & $\begin{array}{l}1.55 \\
0.61 \\
0.56 \\
0.51 \\
0.28 \\
1.90 \\
0.94 \\
1.02\end{array}$ & $\begin{array}{l}14.91 \\
11.55 \\
9.95 \\
7.58 \\
4.15 \\
14.93 \\
13.75 \\
14.98\end{array}$ & 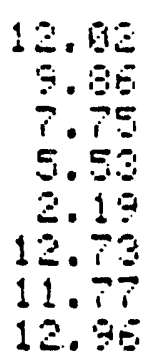 & $\begin{array}{r}19.75 \\
8.58 \\
6.49 \\
4.27 \\
0.93 \\
11.49 \\
10.50 \\
11.89\end{array}$ & 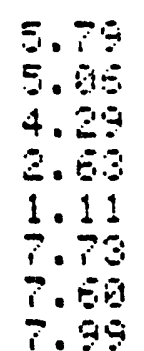 \\
\hline
\end{tabular}


FiL

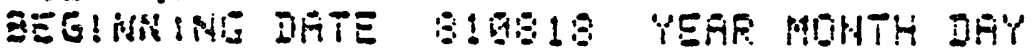

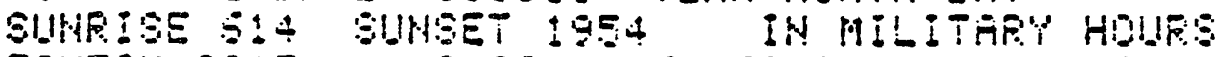

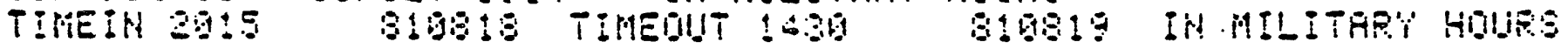

OWEAHIEHT IHCUEATIOH

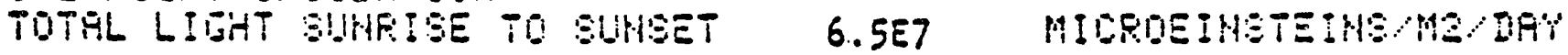

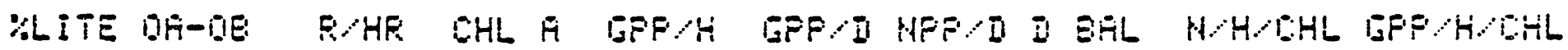

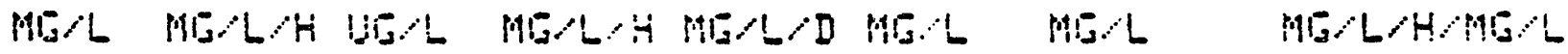

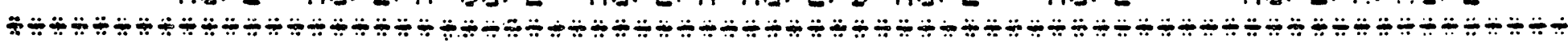

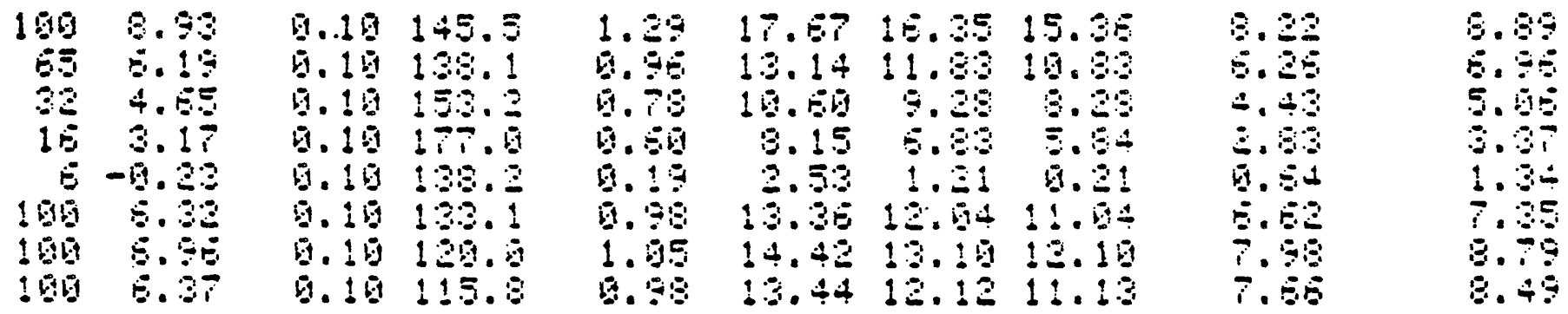


FLEX VA

EEIIHNIMI DATE BDSS22 YEAR MOHTH DH'

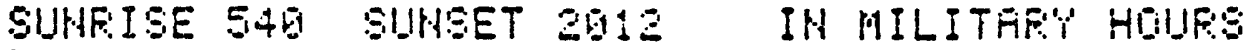

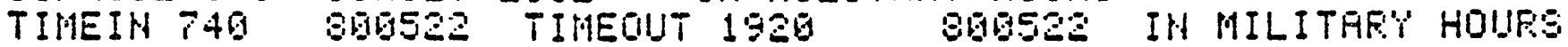

DAY INCUEATIDH ONLY

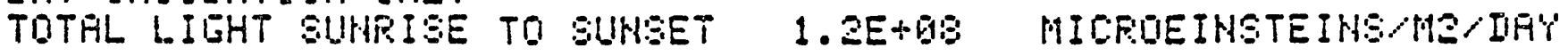

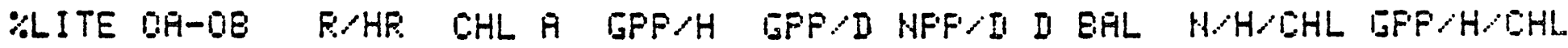

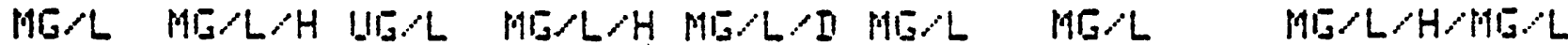

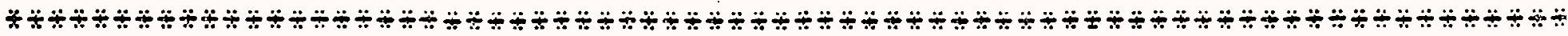

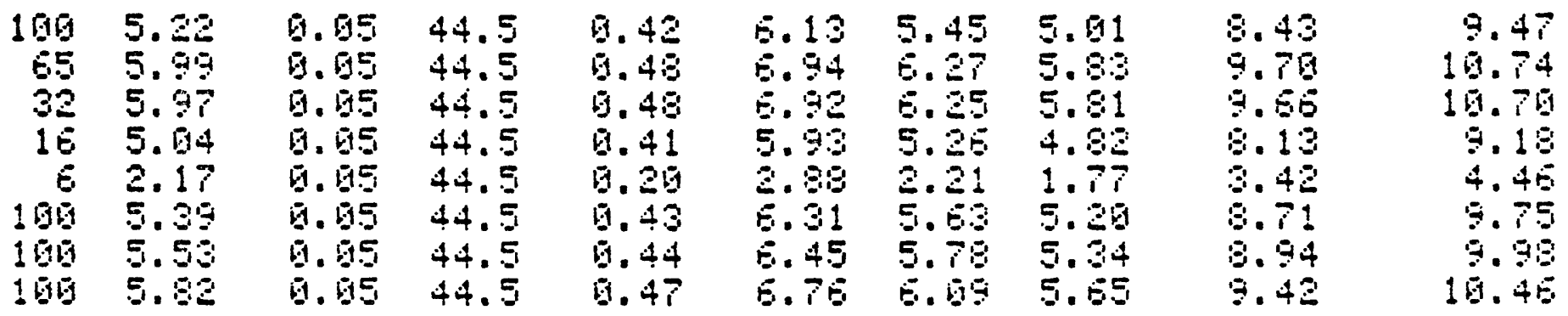

FLEX UA

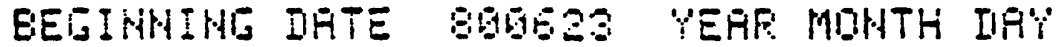

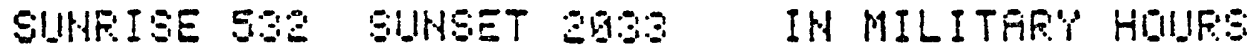

TIMEIN EIIS BGBES TIMEOUT EIGG BOGES IN MILITAFY HOUES

OWEFHIEHT IHDUEATIOH

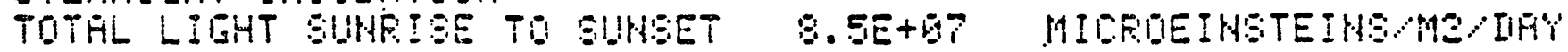

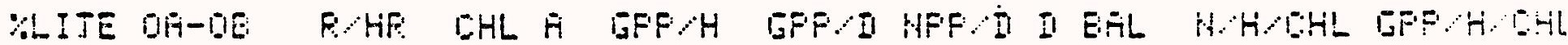

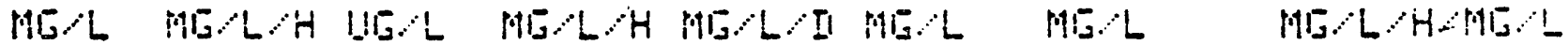
等

\begin{tabular}{|c|c|c|c|c|c|c|c|}
\hline $\begin{array}{rl}105 & 6.35 \\
05 & 5.38 \\
32 & 3.67 \\
16 & 1.45 \\
5 & 9.98 \\
1610 & 5.58 \\
100 & 4.94 \\
100 & 5.50\end{array}$ & 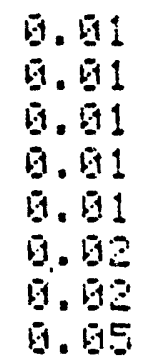 & $\begin{array}{l}52.5 \\
52 \cdot 5 \\
52 \cdot 5 \\
52.5 \\
5.5 \\
5.5 \\
5.5 \\
52.5\end{array}$ & $\begin{array}{l}0.43 \\
0.35 \\
0.25 \\
0.11 \\
0.98 \\
0.30 \\
0.29 \\
6.44\end{array}$ & $\begin{array}{l}5.42 \\
5.48 \\
3.82 \\
1.85 \\
1.20 \\
5.92 \\
4.25 \\
0.55\end{array}$ & $\begin{array}{l}5.25 \\
5.25 \\
3.55 \\
1.52 \\
1.94 \\
5.59 \\
4.97 \\
5.75\end{array}$ & $\begin{array}{l}5.15 \\
5 \cdot 15 \\
3.55 \\
1.42 \\
0.94 \\
5.45 \\
3.91 \\
5.35\end{array}$ & $\begin{array}{l}7.93 \\
5.58 \\
4.53 \\
1.95 \\
1.35 \\
6.10 \\
5.15 \\
7.31\end{array}$ \\
\hline
\end{tabular}


PILEX VA

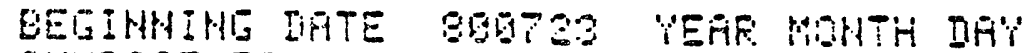

SUHFISE 55 SU SUHSET 2022 IH MILITAFY HOUES

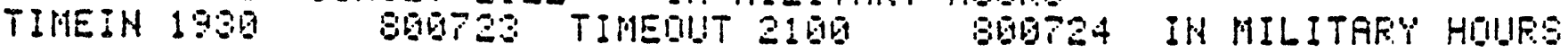

OVEFHIGHT INEUERTIOH

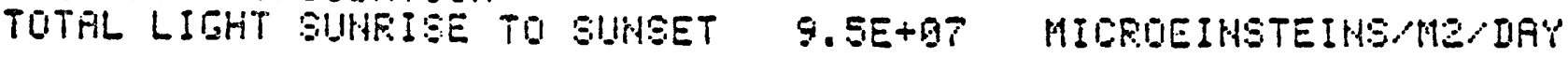

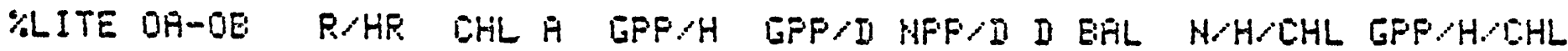

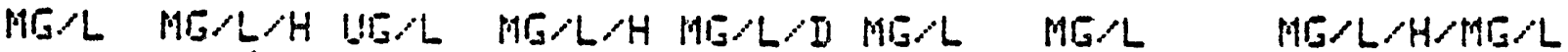

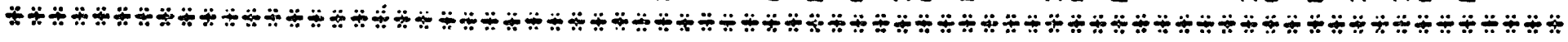

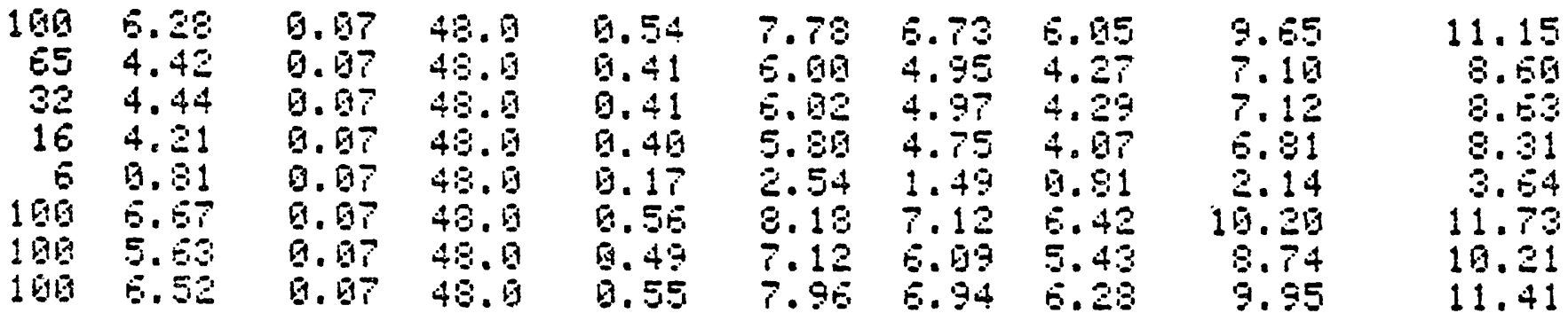

FLEX UP

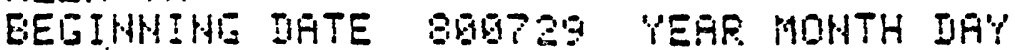

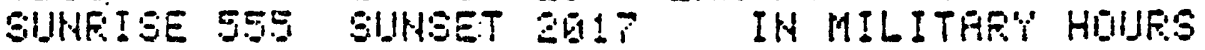

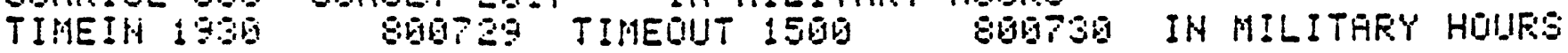

QWEFHIEHT INCUEATIOH

TOTAL LIGHT SUNFISE TO SUHSET 8.3ET MIGFOEIHSTEIHSHE IAY

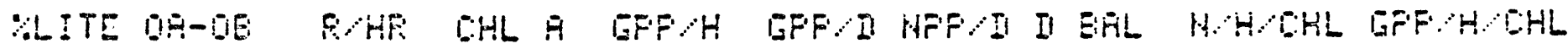

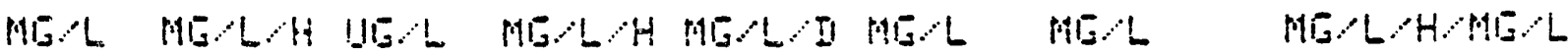

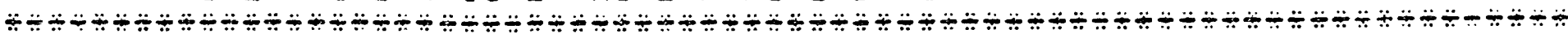

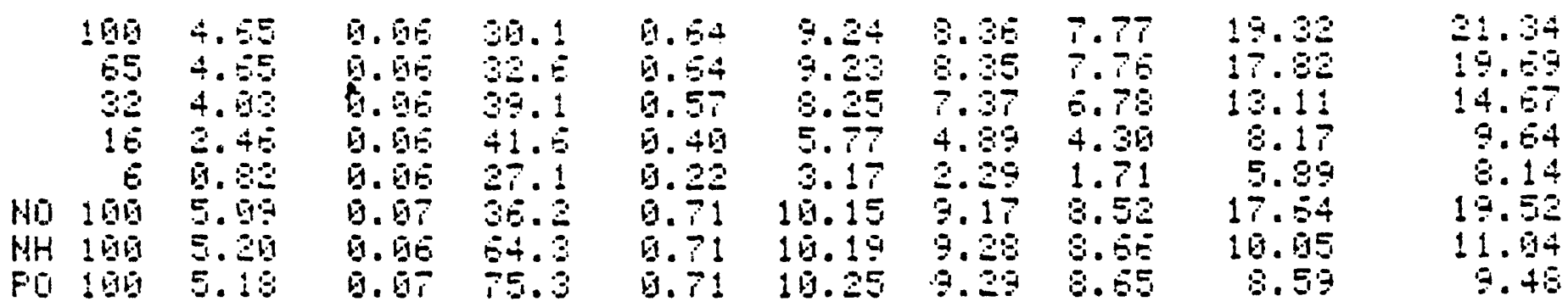


FILEX VA

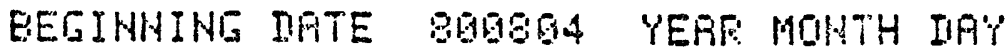

SIHAISE 559 SUHSET 2 G12 IN MILITFFY HOUFS

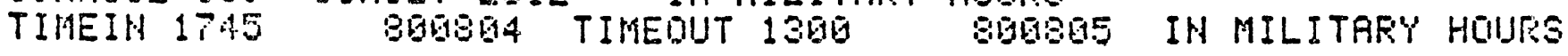

OWERNIGHT IHCUEATION

TOTHL LIGHT SUHRISE TO SUNSET 8.OE7 MICROEINSTEIHS/MZ/DAY

\%LITE OF-OE R/HR CHL A GFF/H GPP/D NPF/D D ERL H/H/CHL GPP/H/CHL

$M G / L$ MG/L/H UG/L MS/L/H MG/L/D ME/L MG/L ME/L/H/MG/L

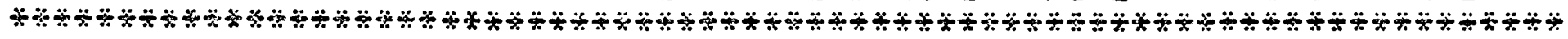

\begin{tabular}{|c|c|c|c|c|c|c|c|c|}
\hline $\begin{array}{r}189 \\
65 \\
32 \\
16 \\
6 \\
190 \\
169 \\
109\end{array}$ & $\begin{array}{r}7.51 \\
6.42 \\
6.86 \\
2.94 \\
-9.20 \\
7.96 \\
7.60 \\
7.74\end{array}$ & $\begin{array}{l}0.09 \\
0.99 \\
0.09 \\
0.09 \\
0.09 \\
0.10 \\
0.10 \\
0.19\end{array}$ & $\begin{array}{l}54.4 \\
54.9 \\
54.4 \\
53.4 \\
41.9 \\
50.4 \\
53.4 \\
58.4\end{array}$ & $\begin{array}{l}1.32 \\
1.16 \\
1.11 \\
0.60 \\
0.22 \\
1.41 \\
1.36 \\
1.37\end{array}$ & $\begin{array}{r}18.70 \\
15.49 \\
15.70 \\
9.44 \\
3.98 \\
19.99 \\
19.33 \\
19.42\end{array}$ & $\begin{array}{r}17.42 \\
15.22 \\
14.49 \\
8.17 \\
1.81 \\
18.58 \\
17.94 \\
18.66\end{array}$ & $\begin{array}{r}16.55 \\
14.35 \\
13.62 \\
7.39 \\
0.94 \\
17.61 \\
10.99 \\
17.13\end{array}$ & $\begin{array}{l}22.54 \\
19.51 \\
13.75 \\
19.77 \\
3.94 \\
25.95 \\
23.64 \\
25.21\end{array}$ \\
\hline
\end{tabular}

ALEX UA

BEGINHIHE IATE SQGESET YEAR MONTH IAYY

SUHFISE EUA SUHSET $200 T$ IN MILITARY HOURS

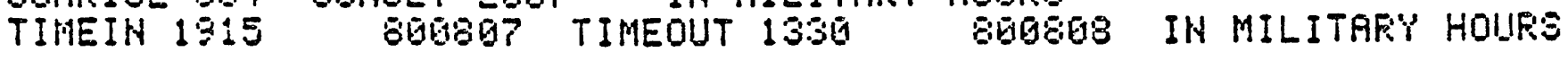

OUERHIEHT INCUEATIOH

TOTHL LIGHT SUHFISE TO SUHSET 1.1E8 MICROEINSTEINS/ME DHY

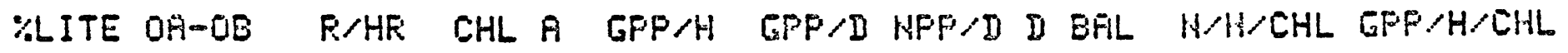

$M G / L$ MG/L/H UE/L MISL/H ME/L/D MG/L MG/L ME/L/H/MG/L

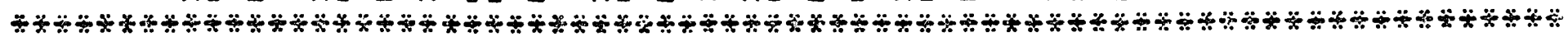

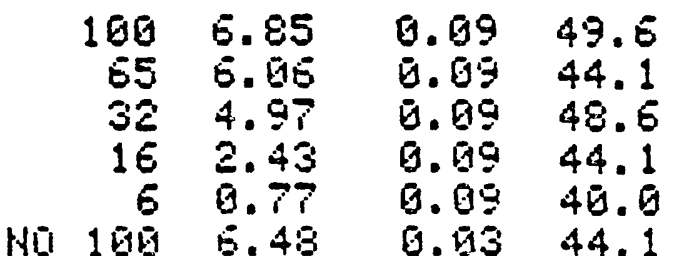

HH 1 19日 $6.91 \quad 0.15 \quad 43.4$

FII 19ि $7.31 \quad 0.11 \quad 45.1$

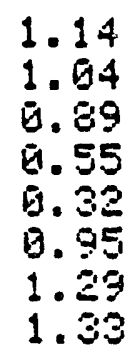

16.0514 .70

14.5513 .29

13.89

12.49

11.23

12.49

7.69

6.43

10.34

4.56

3.29

5.54

13.23

12.96

2.41

13.13

12.55

13.62

15:46 14.60

17.1216 .07
21.20

21.44

16.44

19.37

5.85

20.75

26.36

27.01
23.192

23.48

18.29

12.41

8. 11

21.43

23.74

29.37 
FLEX UA

EEEIHHIHI IATE SUDS13 YEAF MDHTH DFY

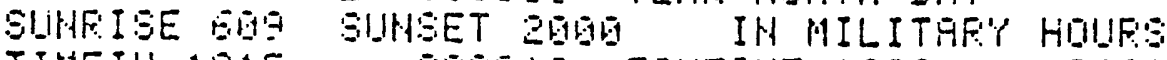

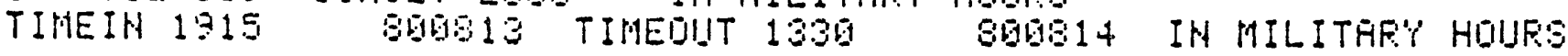

DWEFHILHT IHELEATIOH

TOTHL LIIHT SUHFISE TD SUHSET 1.0E8 MIOFOEIHETEIHE/M2/DFY

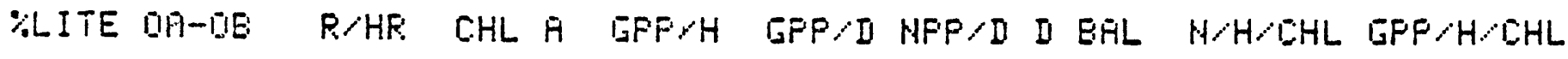

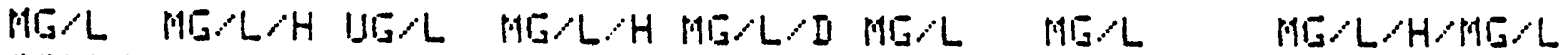

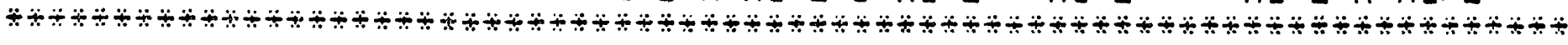

\begin{tabular}{|c|c|c|c|c|c|c|c|c|}
\hline 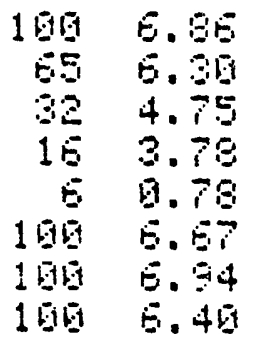 & $\begin{array}{l}0.05 \\
0.05 \\
0.05 \\
0.05 \\
0.05 \\
0.09 \\
0.09 \\
0.69\end{array}$ & 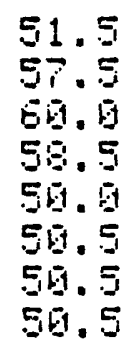 & $\begin{array}{l}1.05 \\
0.93 \\
9.78 \\
0.64 \\
0.24 \\
1.14 \\
1.18 \\
1.10\end{array}$ & $\begin{array}{r}14.74 \\
13.83 \\
19.85 \\
8.93 \\
3.28 \\
15.73 \\
15.96 \\
15.23\end{array}$ & $\begin{array}{r}14.91 \\
12.95 \\
19.95 \\
8.29 \\
2.55 \\
14.45 \\
14.82 \\
13.95\end{array}$ & $\begin{array}{r}13.47 \\
12.42 \\
9.50 \\
7.67 \\
2.92 \\
13.5 \\
13.98 \\
13.92\end{array}$ & $\begin{array}{l}19.64 \\
15.26 \\
12.07 \\
19.12 \\
3.08 \\
20.69 \\
21.19 \\
19.95\end{array}$ & $\begin{array}{l}20.65 \\
17.19 \\
12.95 \\
11.92 \\
4.73 \\
22.59 \\
22.87 \\
21.79\end{array}$ \\
\hline
\end{tabular}

FLEK UA

EEITHAINI DATE 8GGEZU TEAF MONTH IAY

SUHFISE 614 SUHSET 1954 IN MILITAR'T HOURS

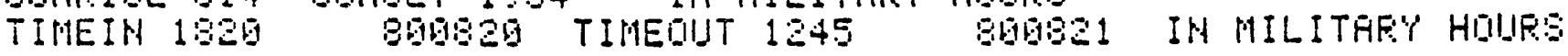

DUEFNILHT INEUEATION

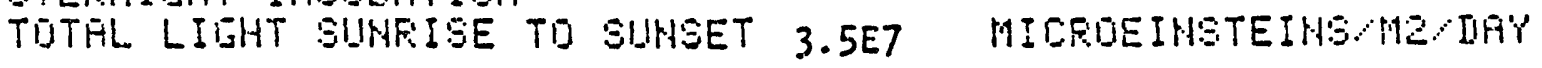

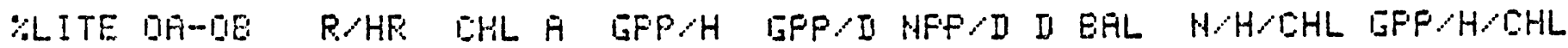

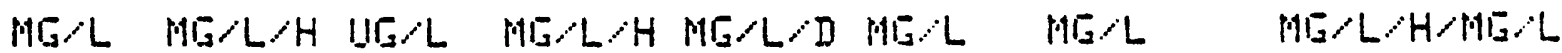

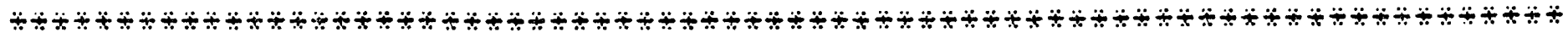

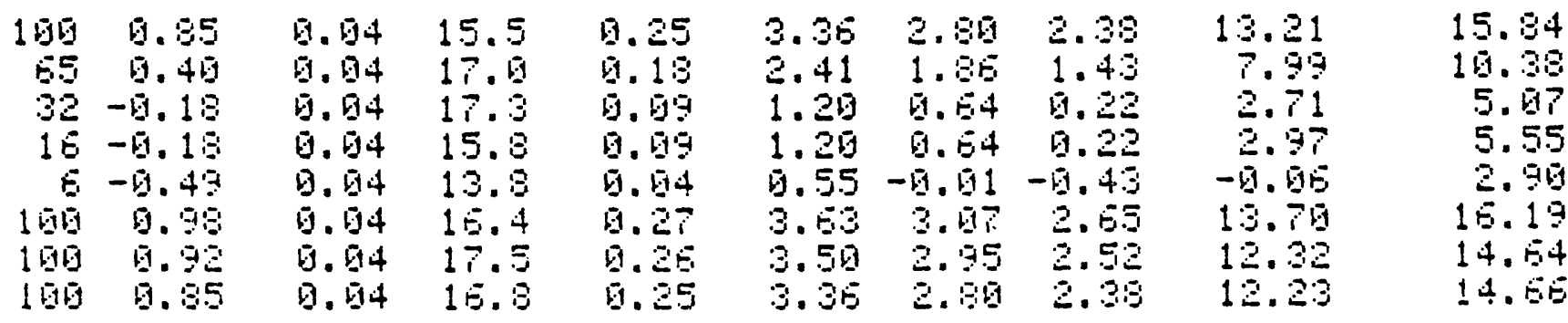


RLEE UA

EELIHHTHE DHTE BEGSES VEAF MOHTH IATY

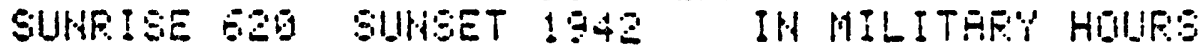

TIMEIN 2145 BDHEZS TIMEOUT 1315 BUGBSE IH MILITAFH HOUES

OWEENITHT INDLERTION

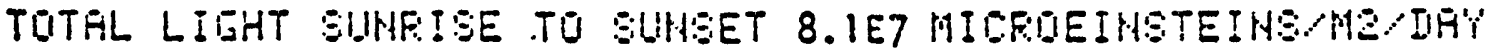

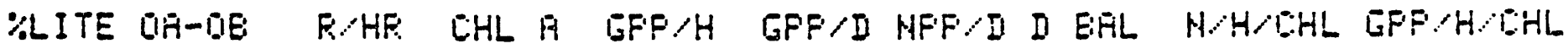

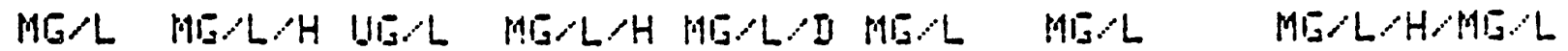

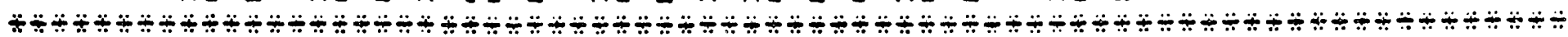

\begin{tabular}{|c|c|c|c|c|c|c|c|c|}
\hline 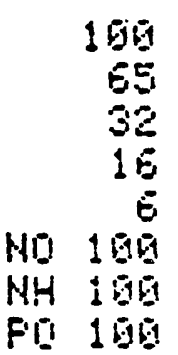 & $\begin{array}{l}1.82 \\
2.09 \\
1.74 \\
1.14 \\
6.13 \\
2.02 \\
3.95 \\
2.17\end{array}$ & $\begin{array}{l}0.93 \\
0.93 \\
0.03 \\
0.93 \\
0.03 \\
0.03 \\
0.03 \\
0.03\end{array}$ & $\begin{array}{l}17.5 \\
19.5 \\
21.5 \\
21.0 \\
15.8 \\
15.6 \\
17.5 \\
17.5\end{array}$ & $\begin{array}{l}9.32 \\
0.30 \\
0.31 \\
0.22 \\
0.93 \\
0.35 \\
0.37 \\
0.39\end{array}$ & 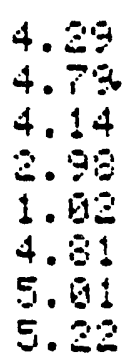 & $\begin{array}{l}3.95 \\
4.45 \\
3.75 \\
2.59 \\
6.56 \\
4.41 \\
4.54 \\
4.76\end{array}$ & $\begin{array}{l}3.57 \\
4.17 \\
3.32 \\
3.30 \\
9.46 \\
4.08 \\
4.17 \\
4.46\end{array}$ & $\begin{array}{r}15.53 \\
17.25 \\
13.83 \\
7.35 \\
3.28 \\
26.50 \\
19.45\end{array}$ \\
\hline
\end{tabular}

ALEX VIT

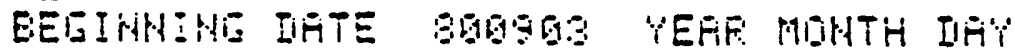

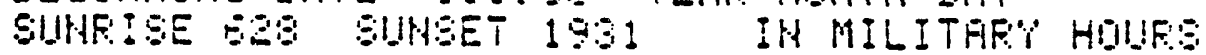

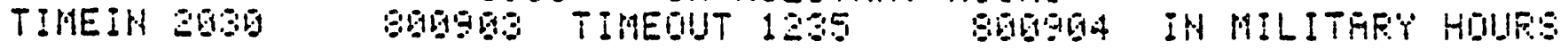

QUEFHIDHT IHELERTIOH

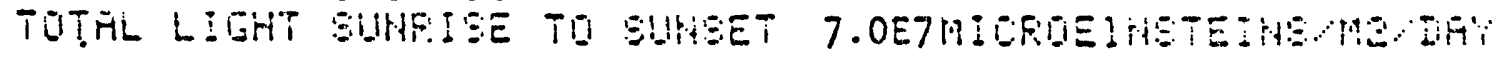

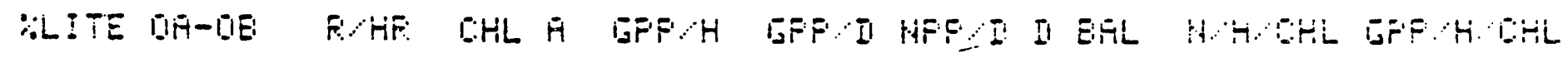

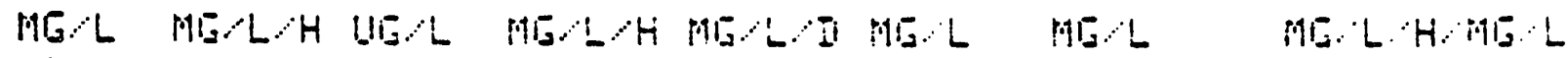

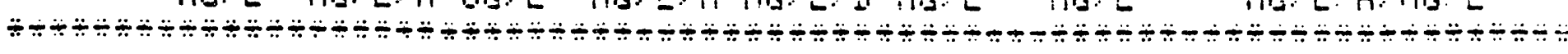

\begin{tabular}{|c|c|c|c|c|c|c|}
\hline $\begin{array}{rr}159 & 2.72 \\
6 & -11 \\
35 & 1.55 \\
16 & 9.78 \\
0 & -9.50\end{array}$ & 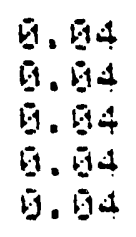 & $\begin{array}{l}23.8 \\
34.5 \\
24.8 \\
31.8\end{array}$ & $\begin{array}{l}9.55 \\
9.45 \\
9.35 \\
9.25 \\
9.92\end{array}$ & $\begin{array}{l}5.19 \\
5.8 \\
4.8 \\
3.95 \\
6.30\end{array}$ & $\begin{aligned} 5.50 \\
5.35 \\
4.15 \\
2.5 \\
-6.21\end{aligned}$ & $\begin{array}{r}5.25 \\
4.92 \\
6.9 \\
2.98 \\
-9.65\end{array}$ \\
\hline
\end{tabular}


FILEY UA

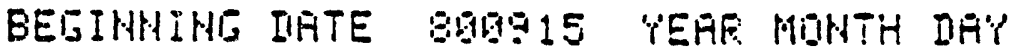

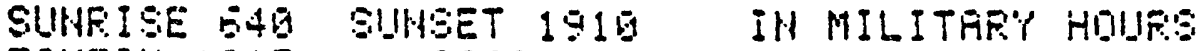

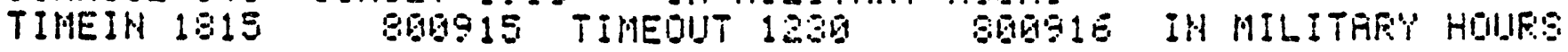

DVERMIEHT IHELERTIDH

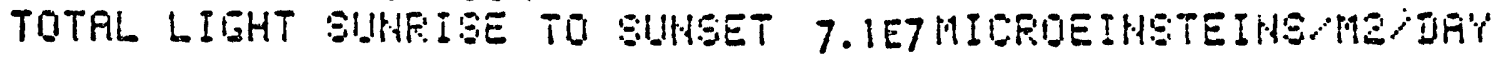

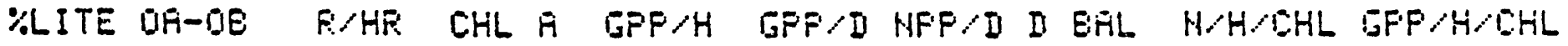

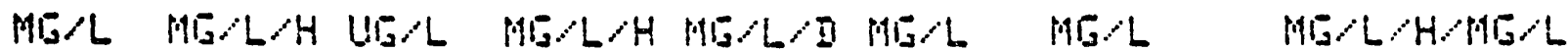

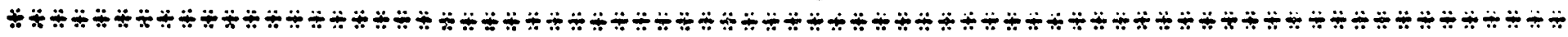

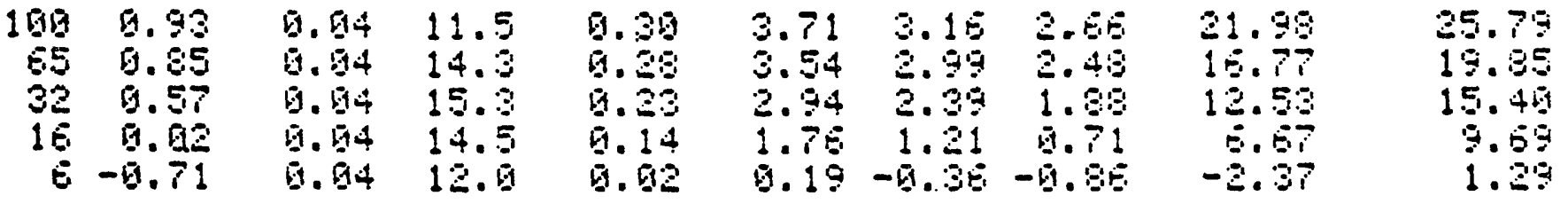

ALEX UA

BEGINHINI IHTE BQ1118 VERF MONTH IAY

SUHFTSE EAS SUHSET IBAO IH MILITFFY HOUFS

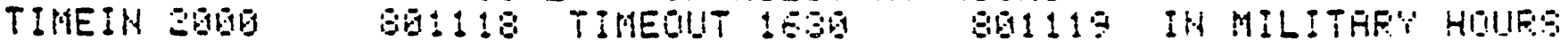

OUEFHIEHT INIOUERTION

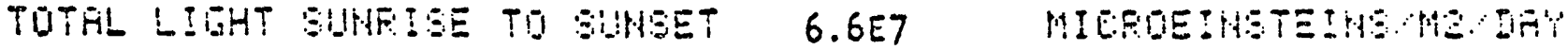

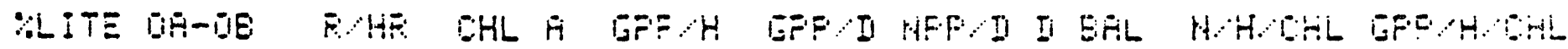

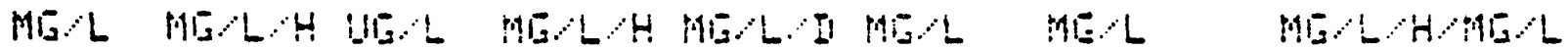

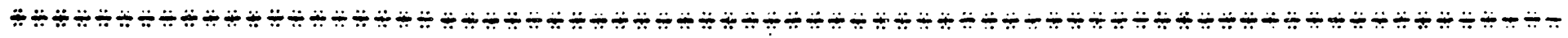

\begin{tabular}{|c|c|c|c|c|c|c|c|c|}
\hline 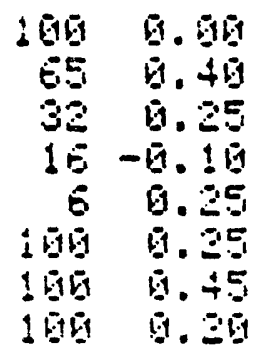 & 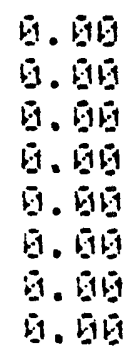 & $\begin{array}{l}11.5 \\
11.6 \\
12.1 \\
12.1 \\
12.5 \\
11.6 \\
11.0 \\
11.0\end{array}$ & 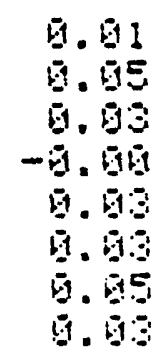 & 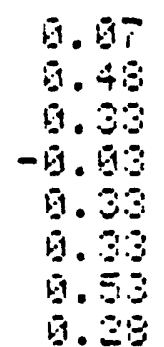 & 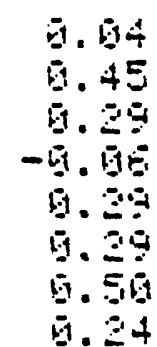 & 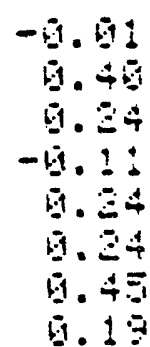 & $\begin{array}{r}3.30 \\
3.96 \\
3.40 \\
-1.54 \\
3.40 \\
3.54\end{array}$ & 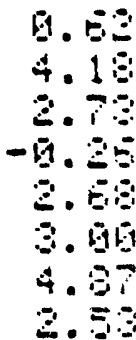 \\
\hline
\end{tabular}


RLES VA

EEGIHNIHT IATE BQ1215 YESE MOHTH IHY

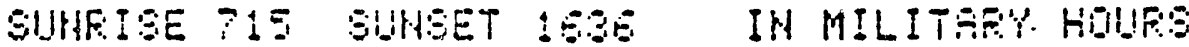

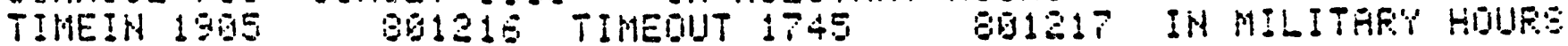

QWEFHIEHT IHELEETTEOH

TOTHL LIGHT SUHFISE TO SHKEET $6.9 E 7$ MICROEIHSTEINSMMEIIHY

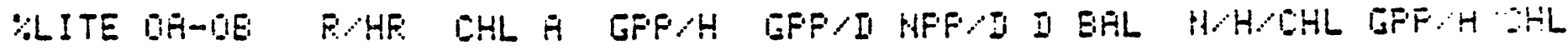

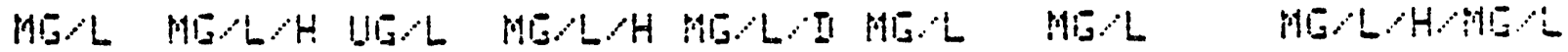

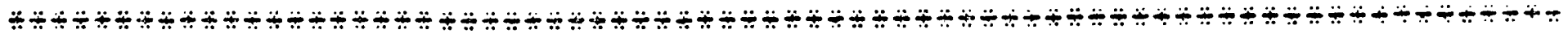

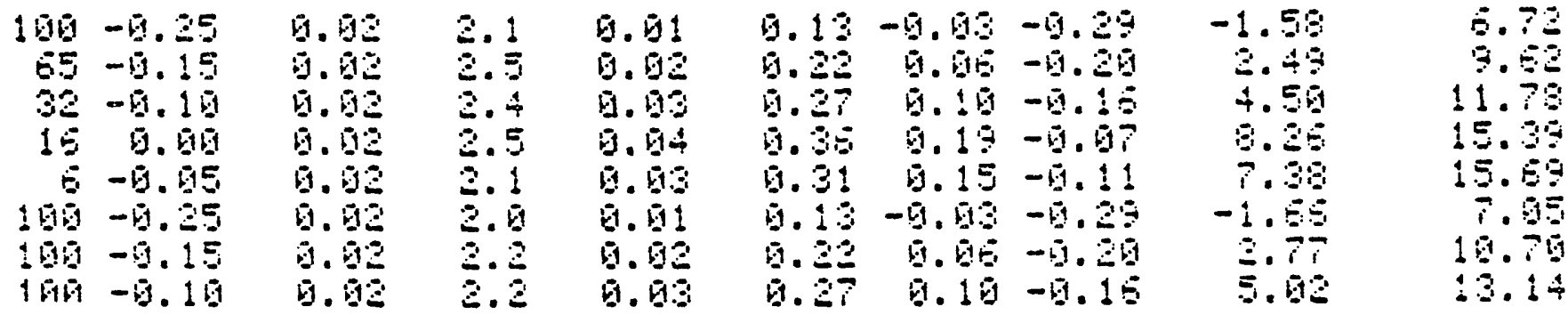

FILEY UF

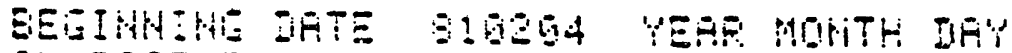

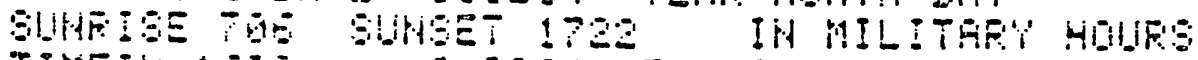

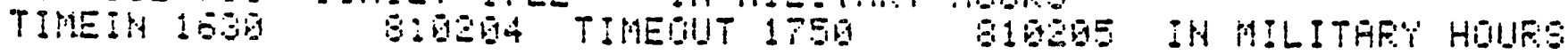

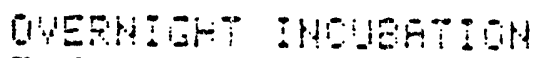

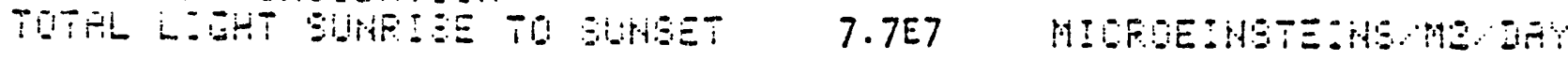

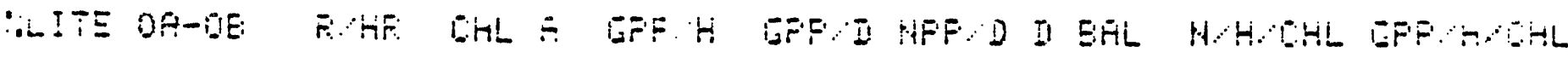

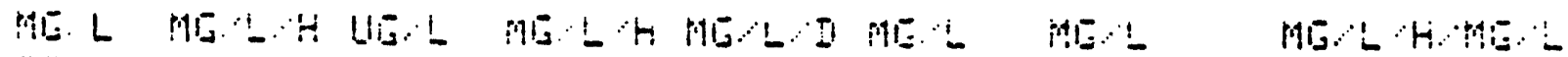

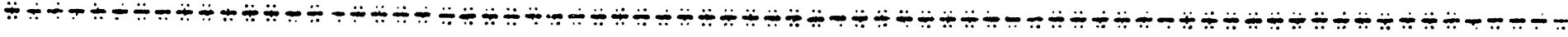

\begin{tabular}{|c|c|c|c|c|c|c|c|}
\hline 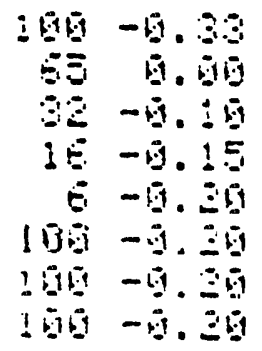 & 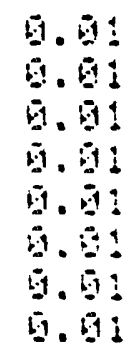 & $\begin{array}{l}3.9 \\
3.0 \\
3.0 \\
3.0 \\
3.0 \\
3.0\end{array}$ & 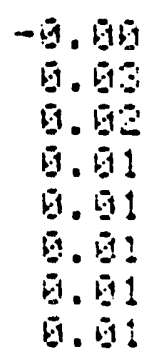 & $\begin{array}{r}-9.98 \\
9.25 \\
0.19 \\
9.14 \\
9.10 \\
6.10 \\
0.15 \\
0.10\end{array}$ & 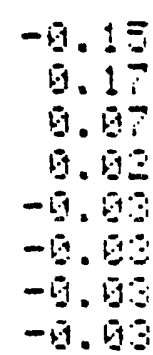 & 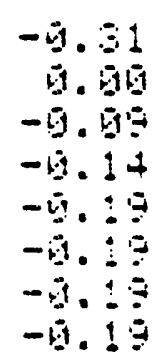 & 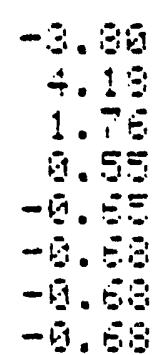 \\
\hline
\end{tabular}


PILEX YA

EEGIHAIHL IATE S1G4G1 TEAR MONTH IFY

SUHFISE 539 SUHSET 1828 IH MILITARY HOUES

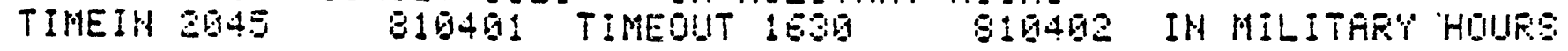

CMERHIGHT IMGUEATION

TOTHL LIGHT SUHFISE TO SUNSET $4.7 E 7$ MICROEIHSTEIHSMM IAT'

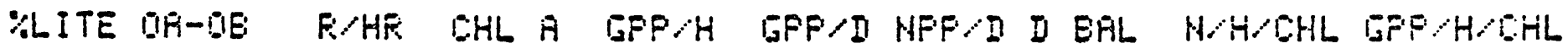

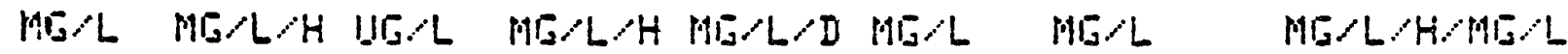

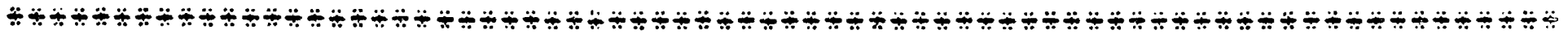

\begin{tabular}{|c|c|c|c|c|c|c|c|}
\hline $\begin{array}{rr}109 & 1.95 \\
65 & 0.59 \\
32 & 0.74 \\
16 & 0.61 \\
5 & 9.19 \\
109 & 0.85 \\
160 & 0.86 \\
100 & 0.85\end{array}$ & $\begin{array}{l}9.92 \\
5.92 \\
0.92 \\
0.92 \\
0.92 \\
6.92 \\
0.92 \\
0.92\end{array}$ & $\begin{array}{l}13.2 \\
14.8 \\
14.2 \\
15.6 \\
13.5 \\
13.7 \\
13.8 \\
12.7\end{array}$ & $\begin{array}{l}0.13 \\
0.13 \\
0.19 \\
9.19 \\
0.65 \\
9.11 \\
0.12 \\
0.11\end{array}$ & $\begin{array}{l}1.65 \\
1.02 \\
1.35 \\
1.17 \\
0.57 \\
1.45 \\
1.49 \\
1.45\end{array}$ & $\begin{array}{l}1.44 \\
1.37 \\
1.68 \\
0.45 \\
0.43 \\
1.23 \\
1.34 \\
1.21\end{array}$ & 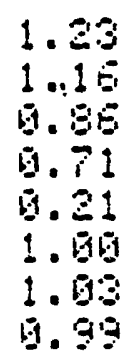 & $\begin{array}{l}2.59 \\
7.39 \\
5.91 \\
4.86 \\
2.47 \\
7.48 \\
7.57 \\
7.41\end{array}$ \\
\hline
\end{tabular}

FLER YA

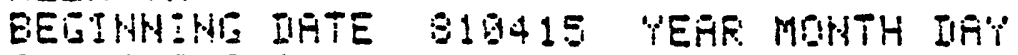

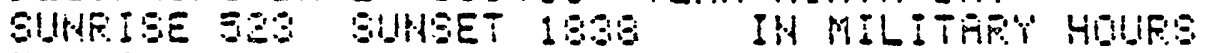

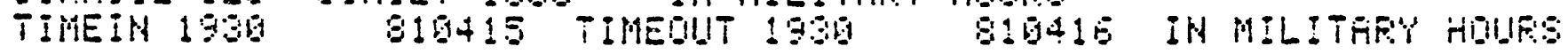

DUEFTIBHT IHE:UEATION

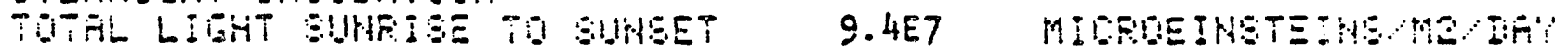

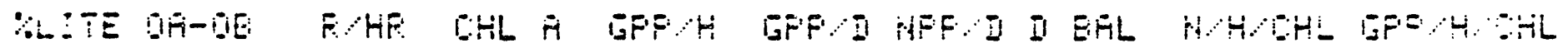

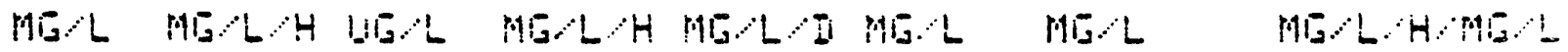

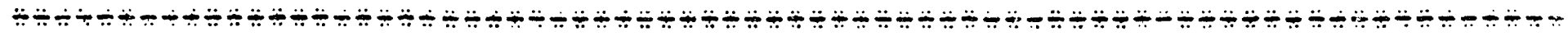

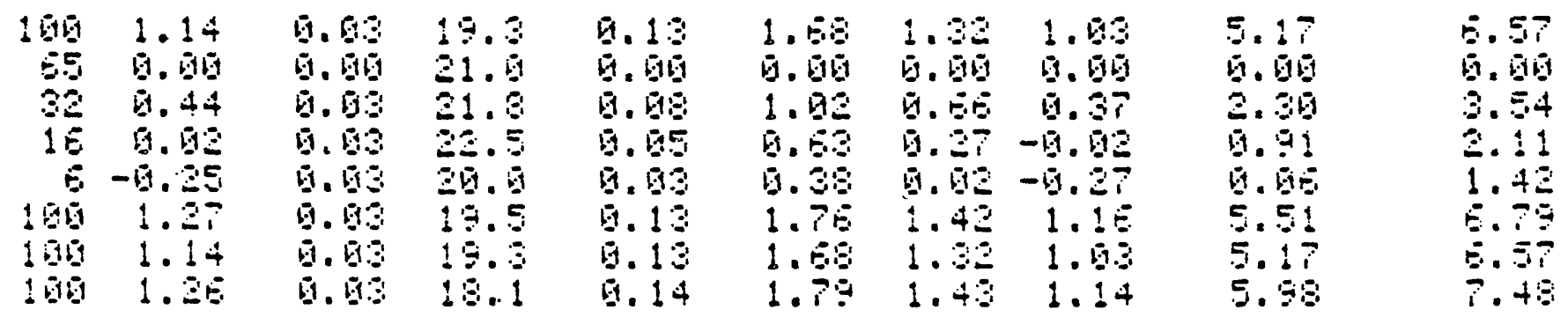


PLEX VA

BEGIHHIHE IHTE 31 GSIG YEAF MOHTH IIAY

SUHFISE 441 SUHSET 1O12 IH MILITAFY HOUF:S

TIMEIH 2000 S1GS1\% TIMEUUT 2045 S10520 IH MILITHRY HOUFS

QVEFHIGHT IHCUEATIOH

TOTHL LIGHT SUHFISE TO SUHSET $8.0 E 7$ MICRGEIHSTEIHSHAIDAY

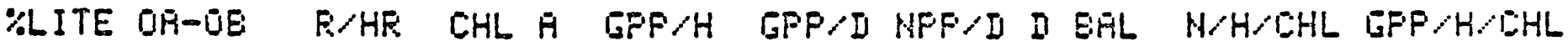

$M G / L$ MG/L/H UG/L MG/L/H MG/L/D MGLL MG/L MG/L/H/MG/L

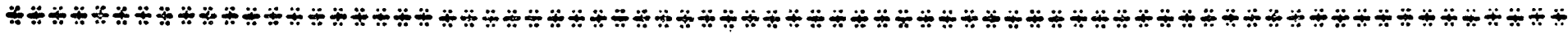

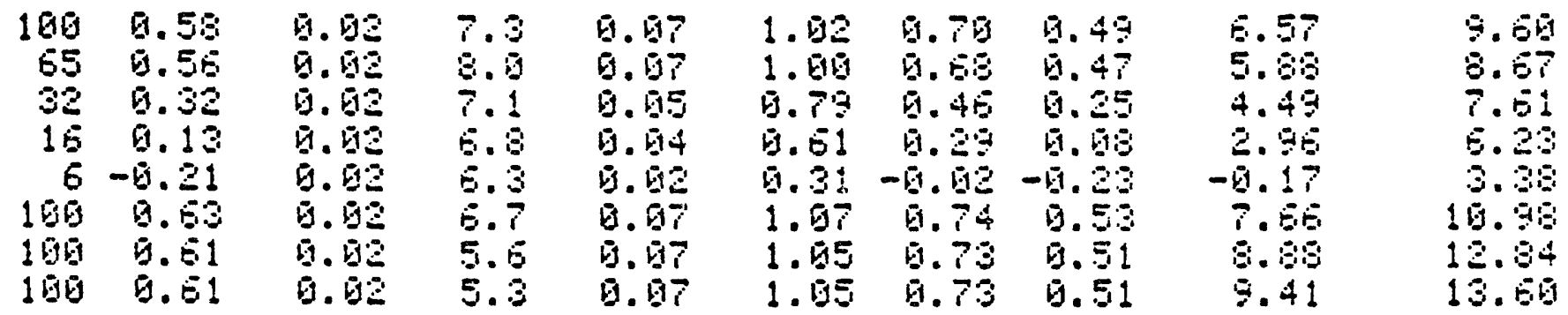

FLER VA

BETIHHIHI IATE BIDSSA TEFR MONTH DAY

SUHFISE 534 SUHSET 2 TOS IH MILITHFY HOUES

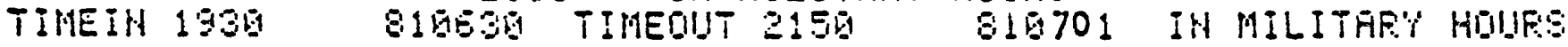

OUEFHISHT IHL:LEATIDH

TOTHL LIEHT SUHEISE TO SUNGET 8.2E7 MICEOEINETETHSMAIIAT

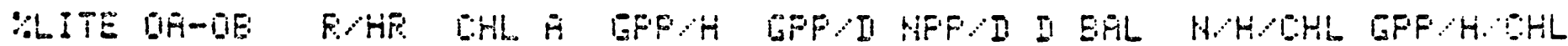

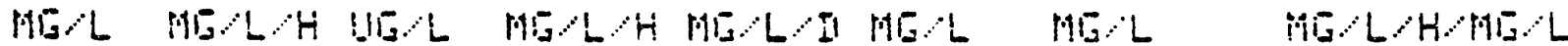

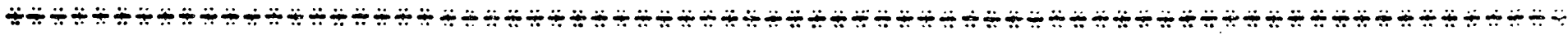

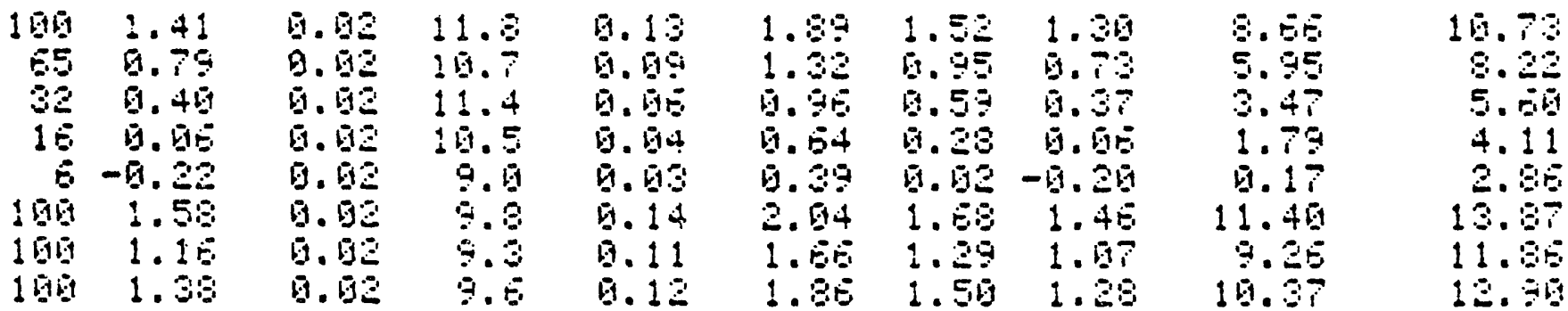


FILEY WA

BEEIHHIHI IATE BIDZBG YEAF MUNTH IIAY

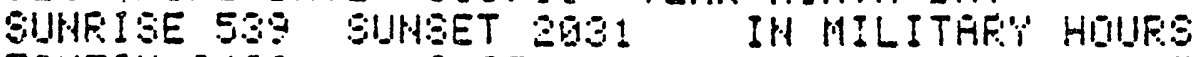

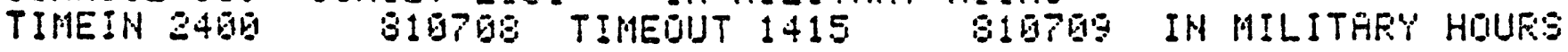

QWEFHIEHT IHULERTIOH

TOTHL LIEHT SUHPISE TU SUHSET I.IEB MICROEIHSTEIHSAME/DAY

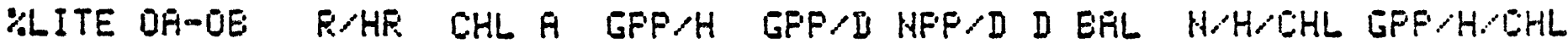

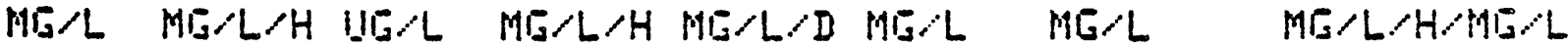

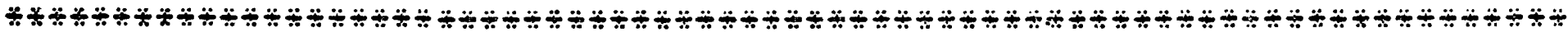

\begin{tabular}{|c|c|c|c|c|c|c|c|}
\hline $\begin{array}{l}4.52 \\
4.43 \\
3.43 \\
2.71 \\
9.58 \\
5.94 \\
4.319 \\
4.75\end{array}$ & $\begin{array}{l}9.55 \\
0.05 \\
0.05 \\
6.05 \\
0.65 \\
0.05 \\
0.05 \\
0.05\end{array}$ & $\begin{array}{l}35.3 \\
34.8 \\
34.0 \\
39.3 \\
47.6 \\
30.2 \\
36.1 \\
30.7\end{array}$ & 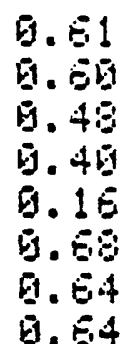 & $\begin{array}{r}9.45 \\
3.96 \\
7.15 \\
5.93 \\
2.42 \\
19.94 \\
9.54 \\
9.47\end{array}$ & $\begin{array}{l}3.31 \\
5.15 \\
5.41 \\
5.18 \\
1.57 \\
9.29 \\
8.98 \\
8.9\end{array}$ & $\begin{array}{l}7.85 \\
7.64 \\
5.34 \\
4.72 \\
1.21 \\
6.83 \\
5.33 \\
6.25\end{array}$ & $\begin{array}{r}15.54 \\
15.74 \\
12.59 \\
5.85 \\
2.51 \\
25.75 \\
15.37 \\
15.18\end{array}$ \\
\hline
\end{tabular}

FILEX VA

EELINHIHI DHTE BIGTZO YERR MONTH IAY'

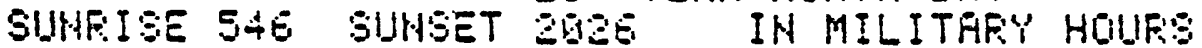

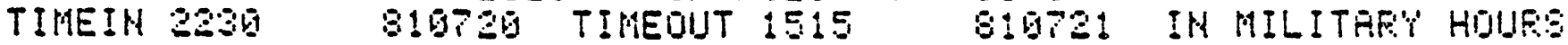

QWEFHIEHT IHIUERTIOH

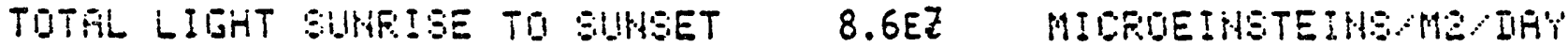

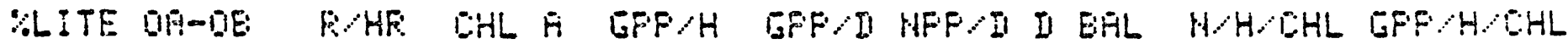

MEL MELAH BEL MELAH MELD MEL MGL MELHAML

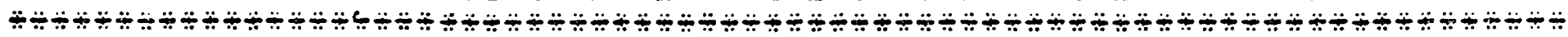

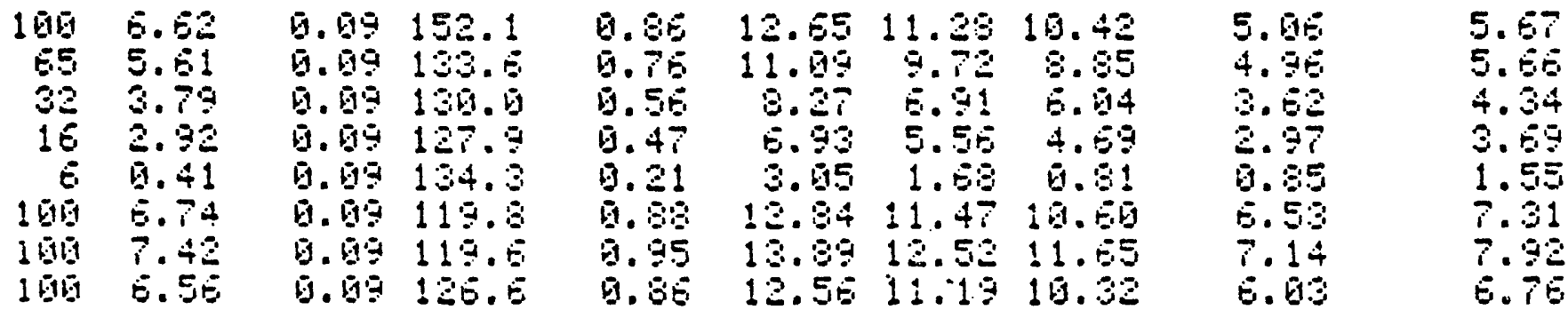


FLER YA

BEGIHNTME IATE 81 GS1S TEAR MONTH IFT'

SUHRISE E14 SUHEET 1954 IN MILITHR'T HDURS

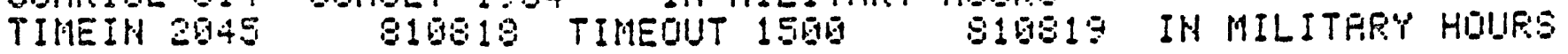

DVERNIEHT INDUEATION

TOTRL LITHT SUHHISE TO SUNSET $6.5 E 7$ MICRDEINSTEINSMMIIAY

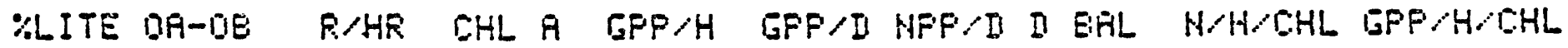

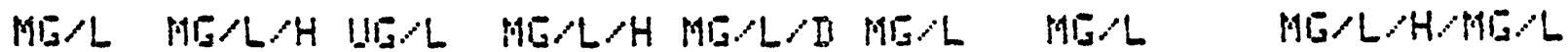

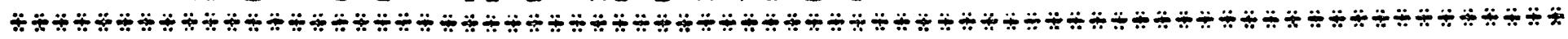

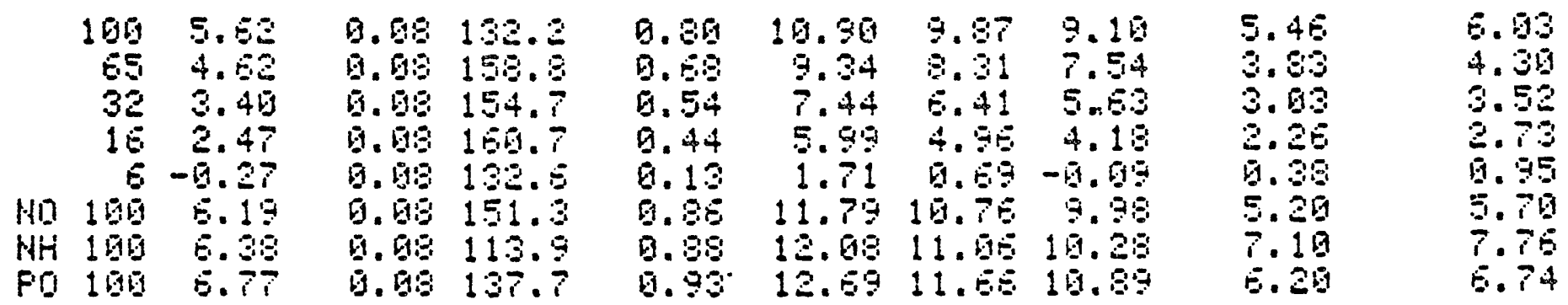

ALEX WH

BEGIHWHE IATE B16825 YERF MONTH DHY

GIHETSE 00 SUNSET 1943 IH MILITAR' HDUFS

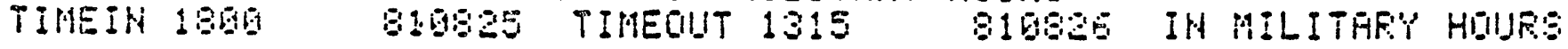

QUEFHISHT IHDUEATIOH

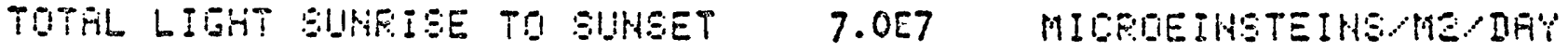

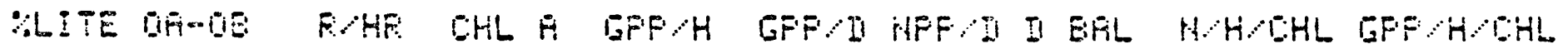

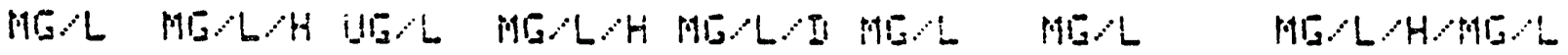

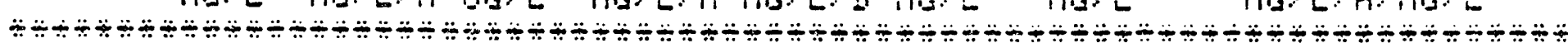

NO 198

160

65

323.27

is 2.79

$6-0.94$

NH 190

PI 1 至
5.98

日. 5

b. 5

日. 58

日.

日.

百.

1. $5:$
55.2

10.8

112.3

147.2

161.8

102.1

91.9

110.5
1.

1. 11

Q. 5

0. 52

1.. 21

9. 35

ด. 97

เ. 95
12.37

13.49

9.17

8.24

2.77

13.12

12.94

12.75
12.3511 .54

12.4611 .65

8.15 7.34

7.20 .41

$1.74 \quad 0.93$

12.1011 .25

11.511 .11

11.7316 .92
G.

8.97

5.42

3.67

1. 28

8. 85

9.5

7.92
10.49

9.70

6.19

4.18

2.

9.

19.52

B. 51 
MT YEEHIN

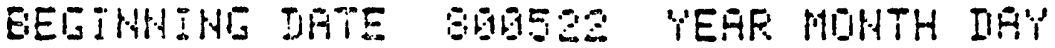

SUHFISE F4O SUHSET 2012 IH MILITHF' HOULS

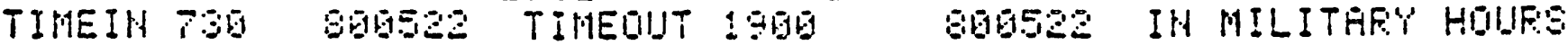

DRY INGEMERTIOHA DHLY

TUTHL LIEHT SUHFISE TO SUHSET 1.2E+QS MIEFIEIHSTEIHSM MATH

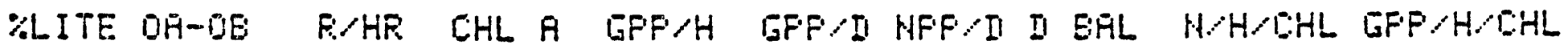

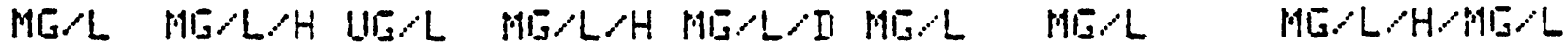

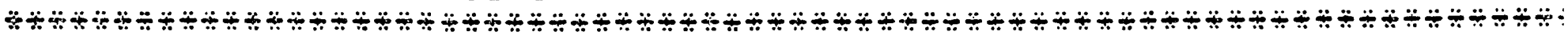

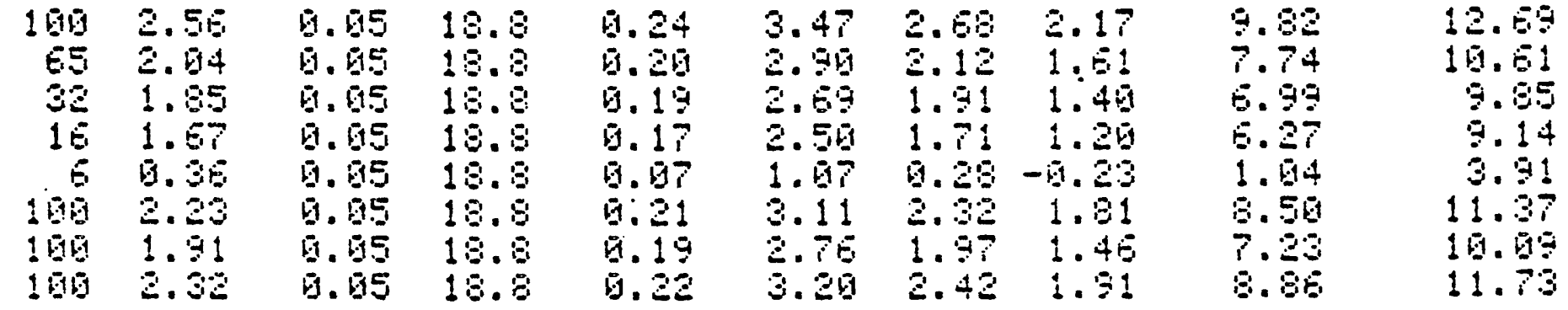

MOLIHT UEFHON

EEEIHHIHE IHTE BGOSZS YEAR MOHTH IHAY

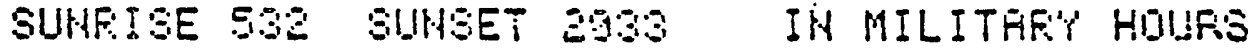

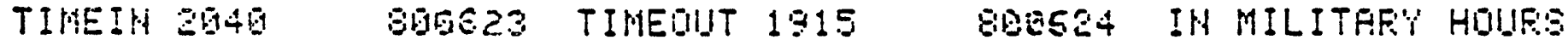

OUEFEYISHT IHIOLEATIOH

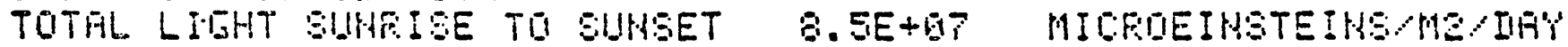

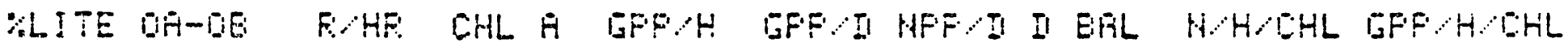

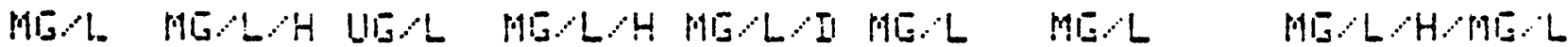

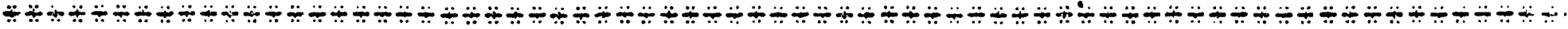

\begin{tabular}{|c|c|c|c|c|c|c|c|c|}
\hline $\begin{array}{r}106 \\
65 \\
35 \\
16 \\
5 \\
160 \\
160 \\
106\end{array}$ & 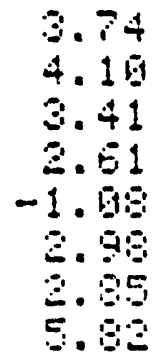 & 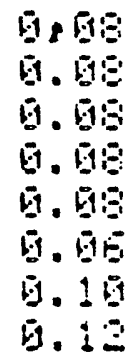 & 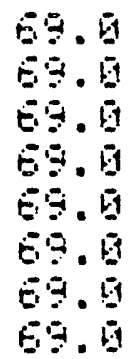 & 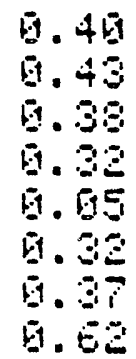 & $\begin{array}{l}5.83 \\
5.47 \\
5.71 \\
4.84 \\
0.81 \\
4.81 \\
5.58 \\
9.87\end{array}$ & $\begin{array}{r}4.87 \\
5.37 \\
4.51 \\
3.54 \\
-6.49 \\
3.87 \\
4.95 \\
7.55\end{array}$ & $\begin{array}{r}4.15 \\
4.55 \\
3.79 \\
2.95 \\
-1.13 \\
3.31 \\
3.19 \\
6.45\end{array}$ & $\begin{array}{r}4.75 \\
5.98 \\
4.85 \\
3.51 \\
-6.30 \\
3.78 \\
3.94 \\
7.39\end{array}$ \\
\hline
\end{tabular}


HATTDH FOIHT

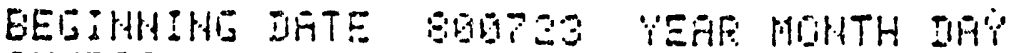

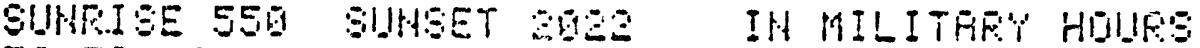

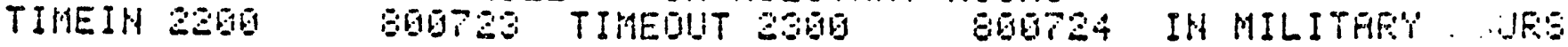

QYEFNIGHT IHCUEATIOH

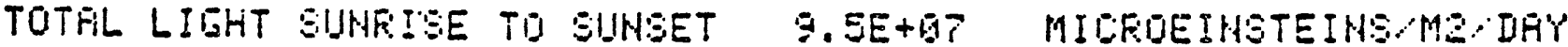

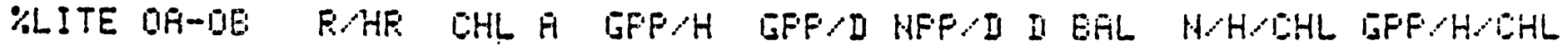

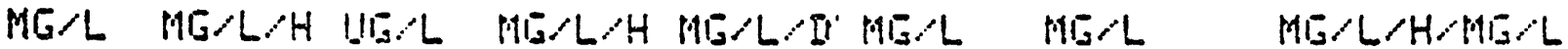

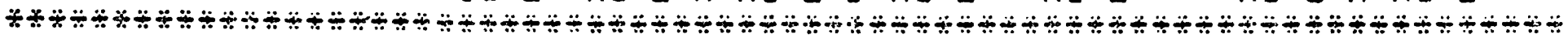

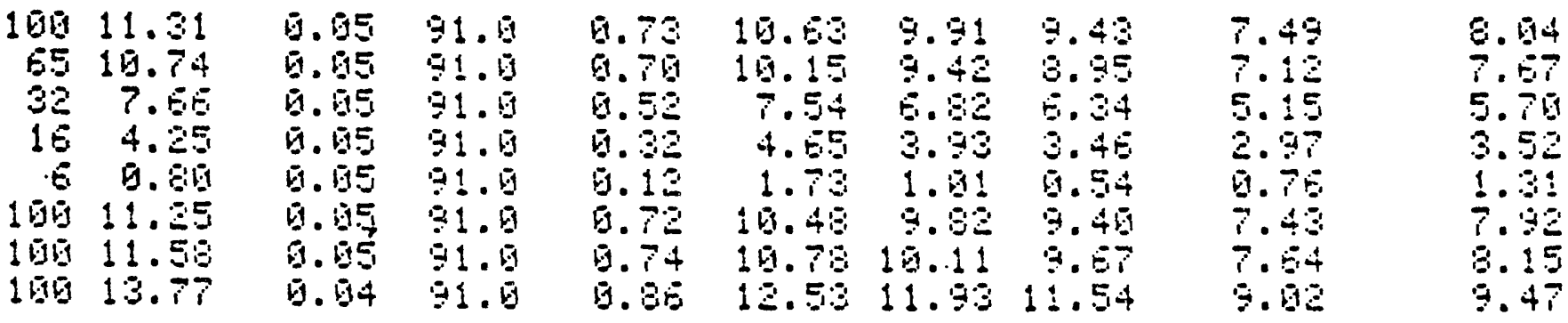

HATTIN FOINT

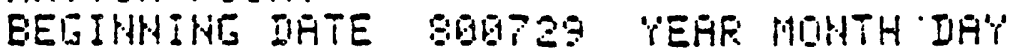

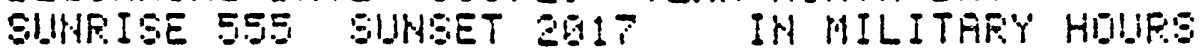

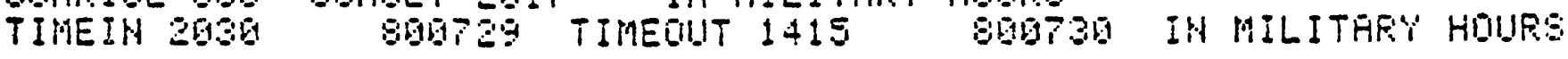

DUEFHIGHT IHEUEATIDH

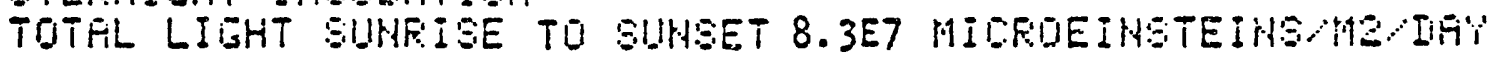

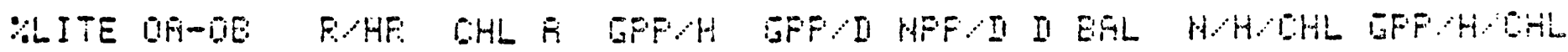

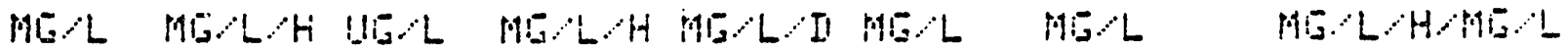

$\ddot{\because}$

\begin{tabular}{|c|c|c|c|c|c|c|c|}
\hline 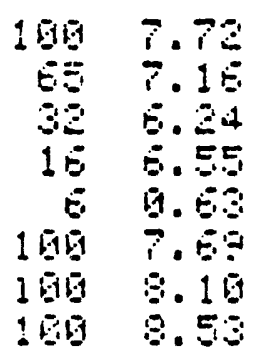 & 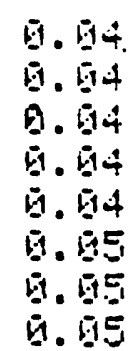 & 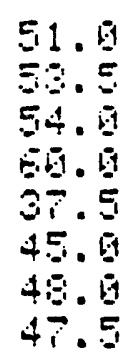 & 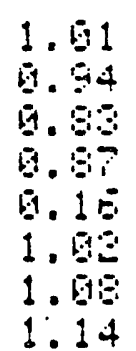 & $\begin{array}{l}14.53 \\
13.50 \\
11.50 \\
12.51 \\
2.30 \\
14.04 \\
15.50 \\
10.30\end{array}$ & 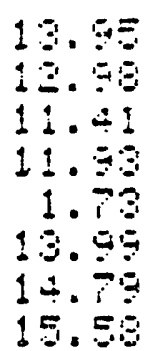 & $\begin{array}{l}15.50 \\
12.59 \\
11.05 \\
12.55 \\
1.54 \\
12.50 \\
14.50 \\
15.65\end{array}$ & 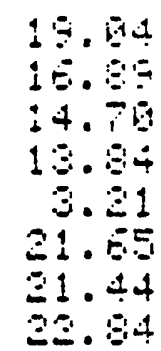 \\
\hline
\end{tabular}


HATTIHA FOIHT

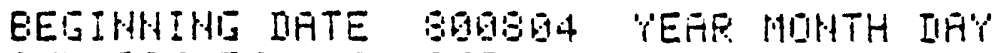

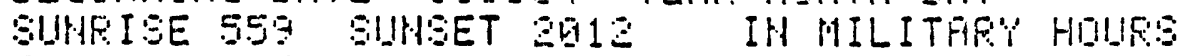

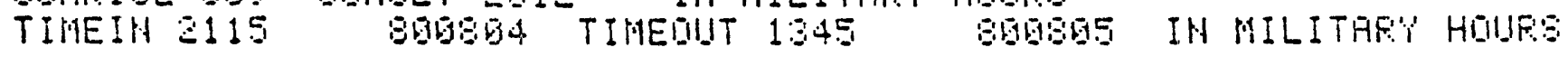

DYEPHISHT IHIOLEATIDH

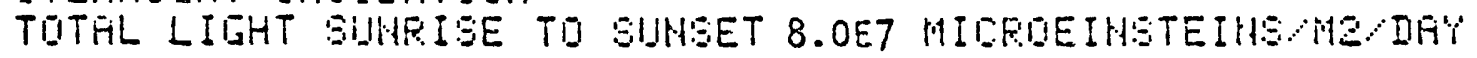

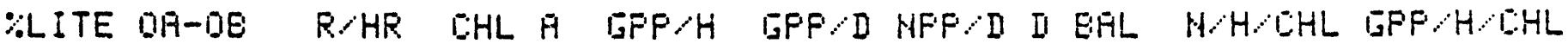

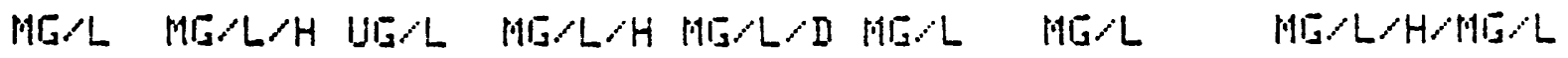

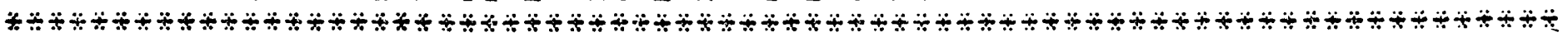

\begin{tabular}{|c|c|c|c|c|c|c|c|}
\hline $\begin{array}{rl}190 & 5.97 \\
65 & 6.30 \\
32 & 5.42 \\
16 & 4.59 \\
5 & 1.20 \\
100 & 5.60 \\
100 & 5.96 \\
160 & 9.12\end{array}$ & $\begin{array}{l}0.04 \\
0.64 \\
0.04 \\
0.04 \\
0.04 \\
0.05 \\
0.05 \\
0.05\end{array}$ & $\begin{array}{l}33.8 \\
33.9 \\
43.6 \\
41.5 \\
37.3 \\
43.3 \\
31.3 \\
51.8\end{array}$ & 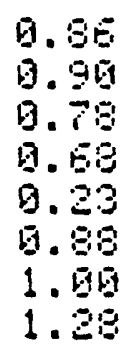 & $\begin{array}{r}12.25 \\
12.73 \\
11.95 \\
3.72 \\
3.35 \\
12.50 \\
14.26 \\
13.23\end{array}$ & $\begin{array}{r}11.71 \\
12.24 \\
16.52 \\
9.19 \\
2.80 \\
11.89 \\
13.49 \\
17.51\end{array}$ & $\begin{array}{r}11.34 \\
11.37 \\
19.15 \\
3.52 \\
2.43 \\
11.40 \\
12.95 \\
17.51\end{array}$ & $\begin{array}{r}21.25 \\
23.23 \\
15.92 \\
15 \cdot 15 \\
5.21 \\
19.32 \\
25.15 \\
24.63\end{array}$ \\
\hline
\end{tabular}

HATTEHA FIIIHT

BEEIHHIHI IATE SBGEGT YERF MUHTH DAY

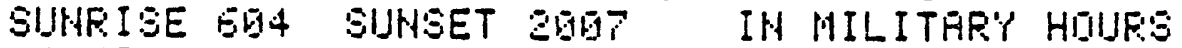

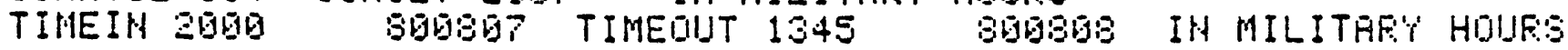

OWEFHIEHT INLUEATION

TUTHL LIEHT SUHAISE TO SUHSET I.IES MIEFDEIHSTEIHSME IIA'

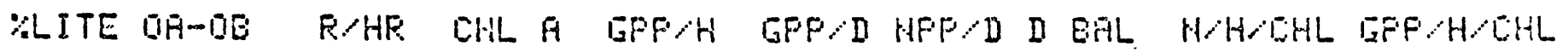

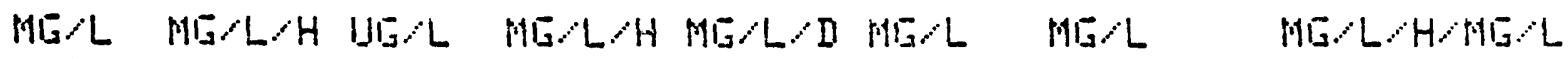
等

\begin{tabular}{|c|c|c|c|c|c|c|c|}
\hline $\begin{array}{rr}105 & 5.52 \\
65 & 7.51 \\
32 & 5.15 \\
16 & 4.44 \\
6 & 1.34 \\
100 & 6.35 \\
160 & 6.90 \\
100 & 9.53\end{array}$ & $\begin{array}{l}5.54 \\
9.54 \\
5.54 \\
6.94 \\
5.54 \\
0.55 \\
0.95 \\
5.11\end{array}$ & $\begin{array}{l}46.4 \\
44.9 \\
51.4 \\
51.9 \\
41.4 \\
35.1 \\
39.9 \\
47.4\end{array}$ & $\begin{array}{l}0.94 \\
1.95 \\
0.89 \\
0.67 \\
0.33 \\
0.94 \\
1.63 \\
1.519\end{array}$ & $\begin{array}{r}13.13 \\
15.13 \\
12.51 \\
9.39 \\
4.53 \\
13.25 \\
14.43 \\
21.67\end{array}$ & $\begin{array}{r}13.54 \\
14.53 \\
11.95 \\
0.53 \\
4.63 \\
13.55 \\
13.50 \\
19.57\end{array}$ & $\begin{array}{r}13.25 \\
14.24 \\
11.57 \\
0.45 \\
3.59 \\
12.54 \\
13.15 \\
13.51\end{array}$ & 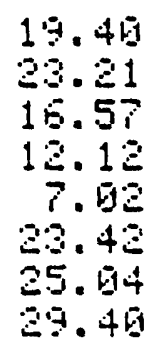 \\
\hline
\end{tabular}


HFiTINA BITEHT

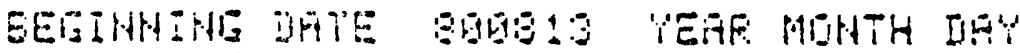

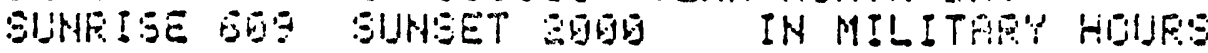

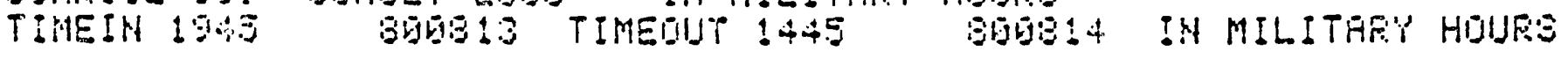

DUERHIEHT IHEUEATIOH

TOTHL LIGHT SUHRISE TO SUMSET 1.OEB MIGFDEIHSTEIHSAMEIIHY

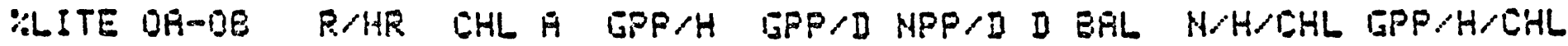

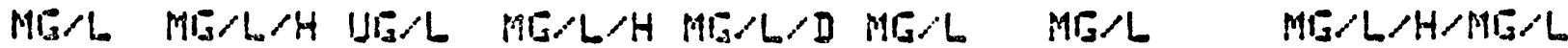

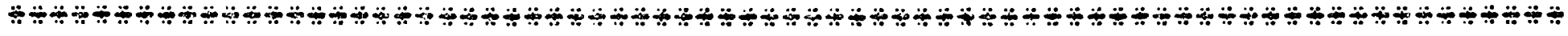

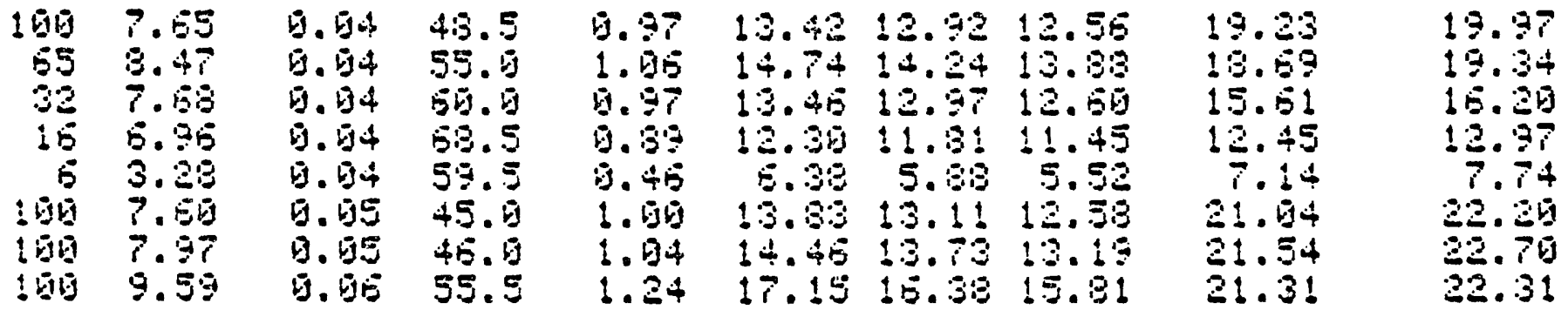

HATTON FIOLT

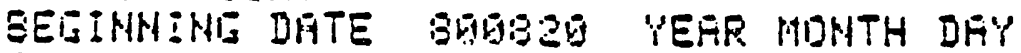

SUHATEE EIT SUNHET 1354 IN MILITFR'Y HOURS

TIHEIH 2315 BDISOS TIMEDUT I

QWEENIEHT IHEJSATIDH

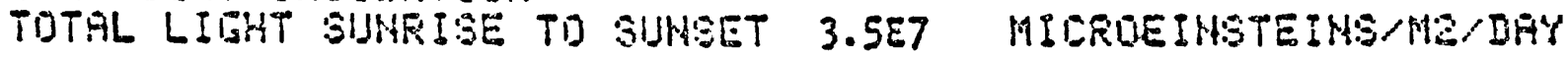

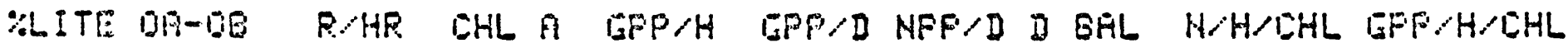

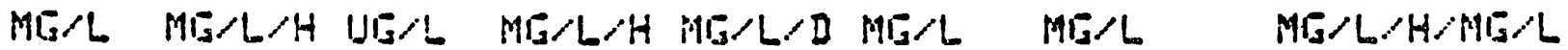

ثب

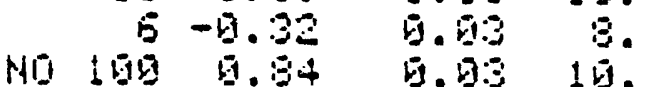

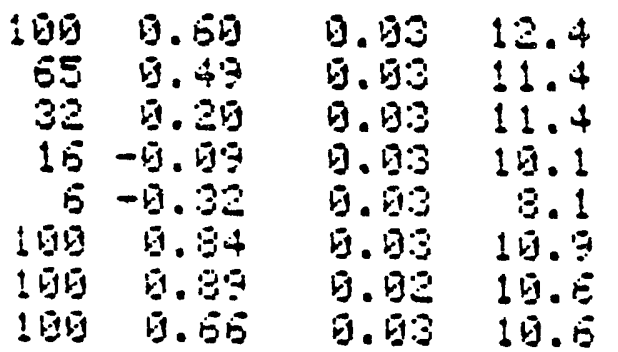

\begin{tabular}{|c|c|c|}
\hline $\begin{array}{l}0.15 \\
0.13 \\
0.09 \\
0.05 \\
0.03 \\
0.13 \\
0.15 \\
0.15\end{array}$ & $\begin{array}{l}1.93 \\
1.78 \\
1.28 \\
9.78 \\
0.37 \\
2.41 \\
2.09 \\
2.199\end{array}$ & $\begin{array}{r}1.53 \\
1.33 \\
0.83 \\
9.31 \\
-9.64 \\
1.95 \\
1.33 \\
1.03\end{array}$ \\
\hline
\end{tabular}

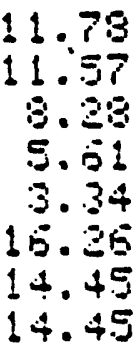


HHTTINH FIIIHT

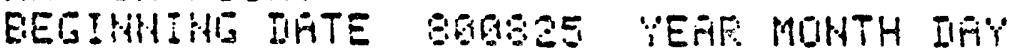

SIHFISE EOH SUHSET 1942 IH MILITRFY HOUFS

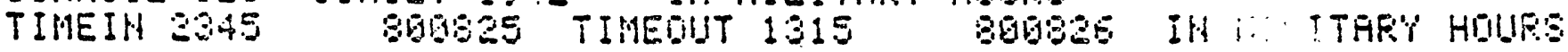

OWEPHIGHT IHEIIEATIDH

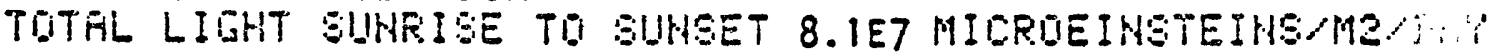

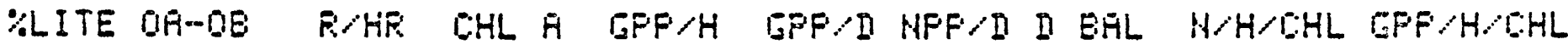

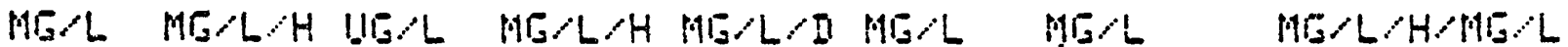

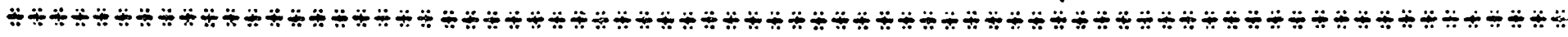

\begin{tabular}{|c|c|c|c|c|c|c|c|}
\hline $\begin{array}{r}180 \\
55 \\
38 \\
16 \\
5 \\
196 \\
150\end{array}$ & $\begin{array}{l}1.41 \\
1.65 \\
1.52 \\
1.05 \\
9.10 \\
1.95 \\
1.56 \\
1.45\end{array}$ & $\begin{array}{l}0.63 \\
6.83 \\
6.93 \\
0.63 \\
0.63 \\
0.63\end{array}$ & $\begin{array}{l}12.0 \\
12.5 \\
13.5 \\
13.5 \\
11.6 \\
11.5 \\
10.5\end{array}$ & $\begin{array}{l}9.27 \\
9.23 \\
6.25 \\
0.23 \\
0.99 \\
0.32 \\
0.32 \\
0.27\end{array}$ & $\begin{array}{l}3.5 \\
3.94 \\
3.79 \\
3.95 \\
1.26 \\
4.25 \\
4.29 \\
3.53\end{array}$ & $\begin{array}{l}3.14 \\
3.51 \\
3.35 \\
2.45 \\
9.75 \\
3.64 \\
3.57 \\
3.21\end{array}$ & $\begin{array}{l}2.75 \\
3.15 \\
3.61 \\
3.13 \\
6.43 \\
6.59 \\
3.57\end{array}$ \\
\hline
\end{tabular}

HATTOH FOIHT

EEGIHHIHE IRTE 8 SGSGS TEAR MONTH IFY

SUHAZEE EO SUNSET 1931 IN MILITARY HOURS

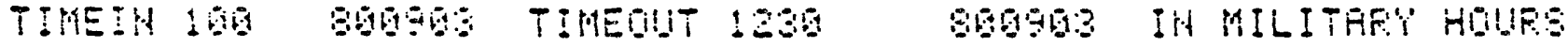

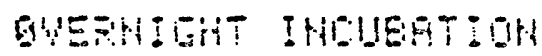

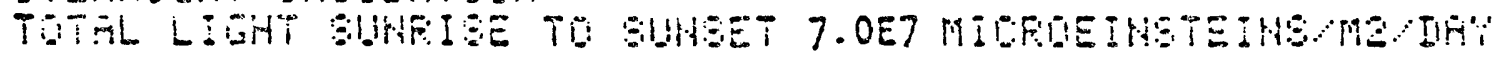

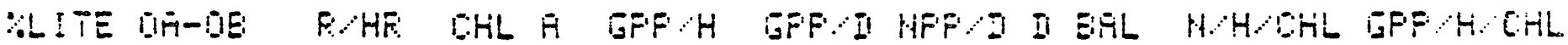

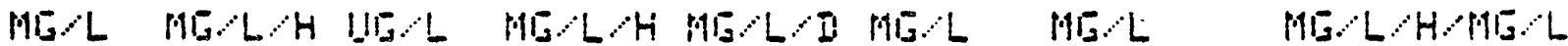
定

\begin{tabular}{|c|c|c|c|c|c|c|c|}
\hline 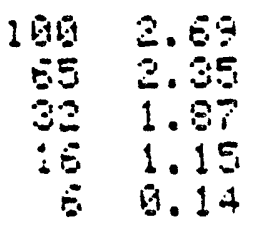 & 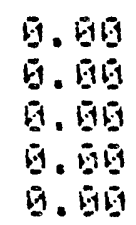 & $\begin{array}{l}17.5 \\
19.5 \\
-15.5 \\
13.5 \\
14.5\end{array}$ & $\begin{array}{l}0.45 \\
9.49 \\
6.32 \\
6.25 \\
6.53\end{array}$ & $\begin{array}{l}5.95 \\
5.17 \\
4.13 \\
2.57 \\
6.39\end{array}$ & $\begin{array}{l}5.55 \\
5.13 \\
4.65 \\
2.53 \\
6.34\end{array}$ & $\begin{array}{l}5.95 \\
5.69 \\
4.65 \\
2.45 \\
6.31\end{array}$ & 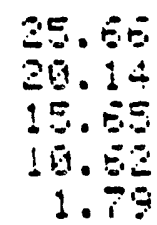 \\
\hline
\end{tabular}


HATTON FOINT

EELIHHIHE IHTE BGIY 15 VEAF MOHTH IFY'

SUNFISE S4D SUHAET 1919 IH MILITAP'Y HOUFS

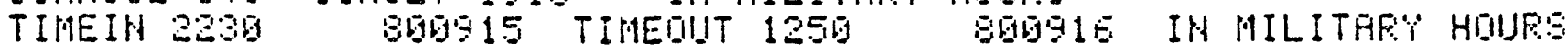

QUEFHILHT IMEILERTIOH

TOTFL LILHT SUHFISE TD SUHSET 7.1E7 MIEFUEIHSTEIHSAMUH'T

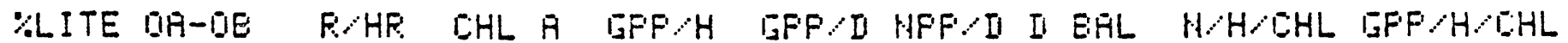

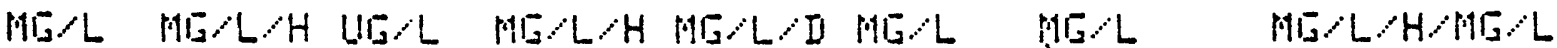

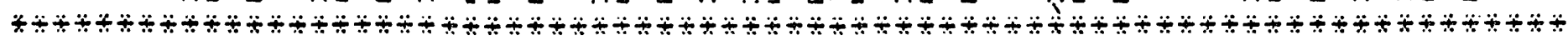

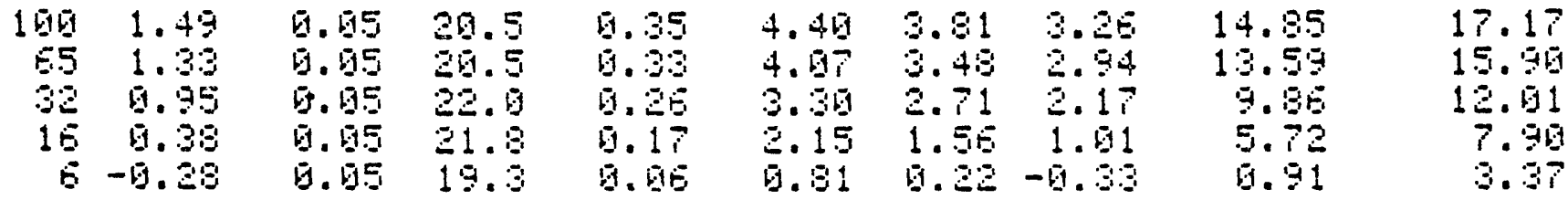

HATTOH FIIHT

EEEINHIHI DFTE BDI11S TEFF MDHTH IFY

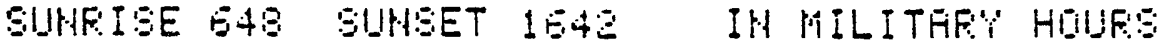

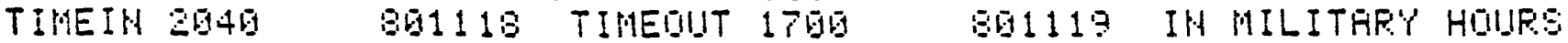

DUEFHIDHT IHLUEATIOH

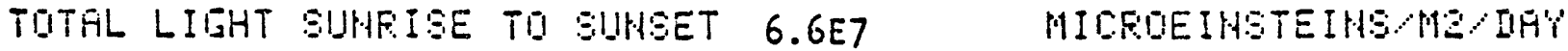

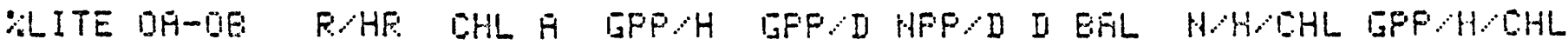

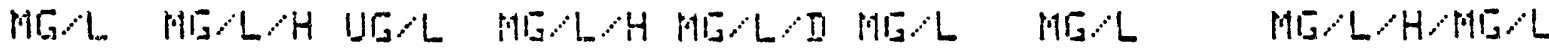

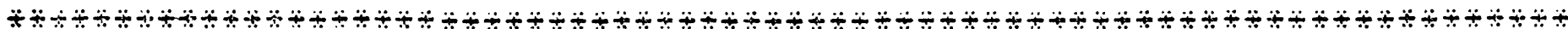

\begin{tabular}{|c|c|c|c|c|c|c|c|c|}
\hline 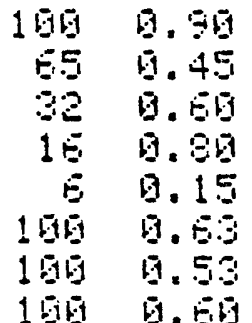 & 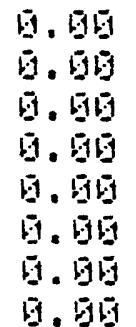 & $\begin{array}{l}9.5 \\
9.5 \\
9.5 \\
9.8 \\
9.5 \\
9.8 \\
9.5 \\
9.5\end{array}$ & 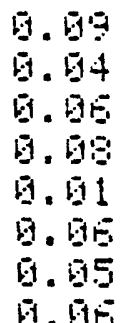 & $\begin{array}{l}0.87 \\
0.44 \\
0.58 \\
0.78 \\
0.15 \\
0.01 \\
0.51 \\
0.58\end{array}$ & $\begin{array}{l}0.87 \\
6.44 \\
0.58 \\
0.78 \\
0.15 \\
0.61 \\
0.51 \\
5.5\end{array}$ & $\begin{array}{l}0.87 \\
6.44 \\
0.58 \\
6.78 \\
0.15 \\
6.51 \\
6.51 \\
0.5\end{array}$ & $\begin{array}{l}9.15 \\
4.5 \\
6.13 \\
6.13 \\
1.53 \\
5.43 \\
5.41\end{array}$ & $\begin{array}{l}9.19 \\
4.8 \\
5.13 \\
8.12 \\
1.5 \\
6.43\end{array}$ \\
\hline
\end{tabular}


HATTOH POINT

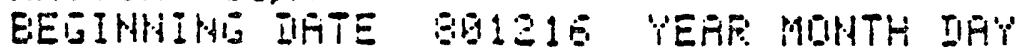

SUHAISE T15 SUHSET 1830 IH MILITRPY HOHES

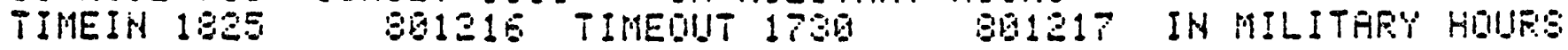

QVERHIGHT INOUEATIOH

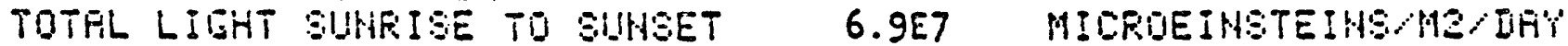

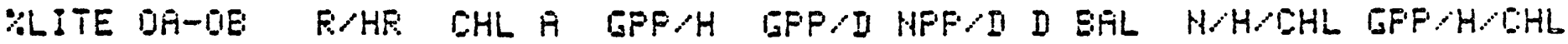

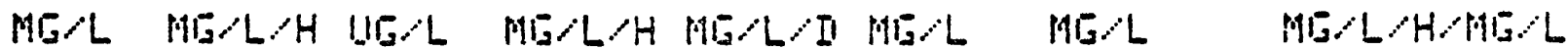

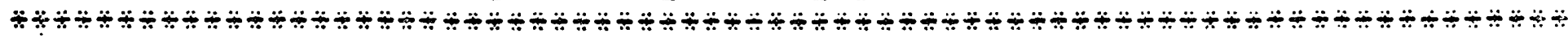

\begin{tabular}{|c|c|c|c|c|c|c|c|}
\hline 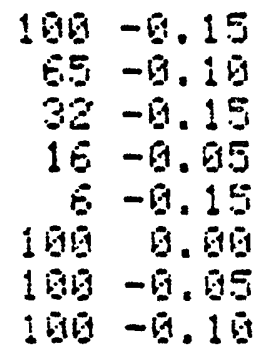 & 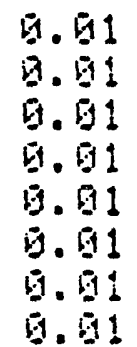 & $\begin{array}{l}2 \cdot 5 \\
3 \cdot 2 \\
2 \cdot 1 \\
2 \cdot 5 \\
2 \cdot 5 \\
3 \cdot 1 \\
3 \cdot 1 \\
3 \cdot 2\end{array}$ & 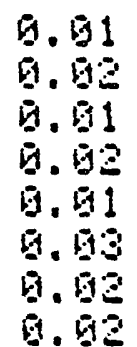 & $\begin{array}{l}0.14 \\
0.19 \\
0.14 \\
0.23 \\
0.14 \\
0.27 \\
0.23 \\
0.13\end{array}$ & $\begin{array}{l}0.92 \\
6.95 \\
0.92 \\
6.11 \\
0.19 \\
6.15 \\
0.11 \\
0.65\end{array}$ & 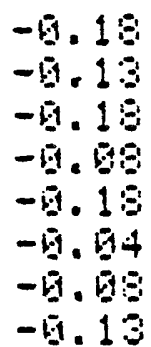 & $\begin{array}{l}5.91 \\
3.93 \\
6.77 \\
4.14 \\
6.94 \\
7.34 \\
5.35 \\
3.415\end{array}$ \\
\hline
\end{tabular}

HATTOH FIINT

EEGIHHIHI DATE Q19204 VEAR MOHTH IAY

SUHFTSE THE SUHSET 1722 IH MILITHFY HOUFS

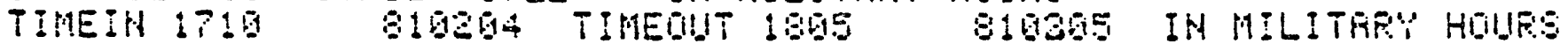

GWEPHIEHT INDUERTIDH

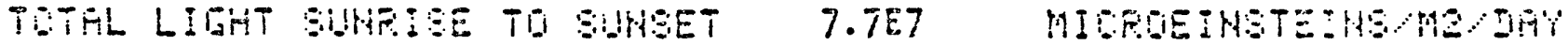

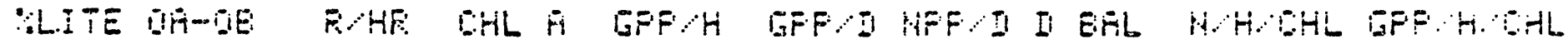

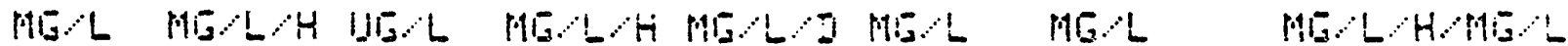

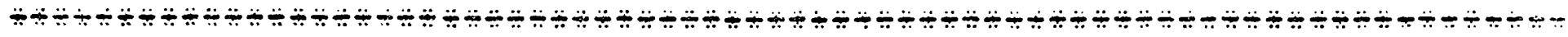

\begin{tabular}{|c|c|c|c|c|c|c|c|}
\hline 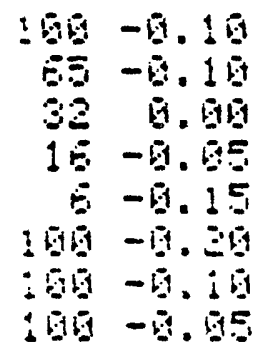 & 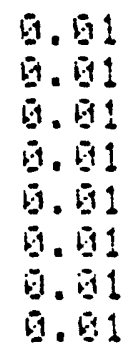 & 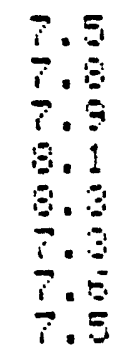 & 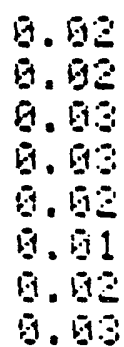 & $\begin{array}{l}5.34 \\
6.24 \\
0.34 \\
9.24 \\
6.25 \\
0.15 \\
6.24 \\
6.24\end{array}$ & 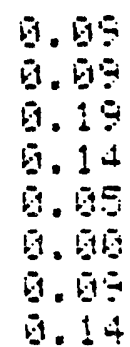 & 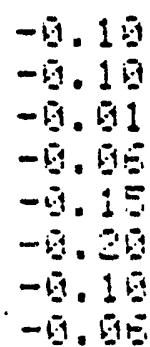 & $\begin{array}{l}1.23 \\
1.13 \\
2.30 \\
1.79 \\
4.50 \\
0.93 \\
1.21 \\
1.34\end{array}$ \\
\hline
\end{tabular}


HRTTIN FOTHT

BEEIHHIHE IHTE BIBADI YERE MINTH IRY

SUNFISE SS\% SUNSET 1 SOS IN MILITHFY HIUFS

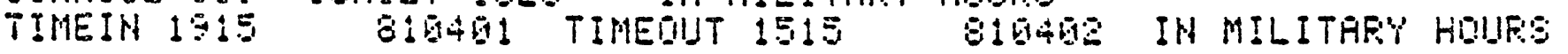

DYEFHISHT IHEUEHTIOH

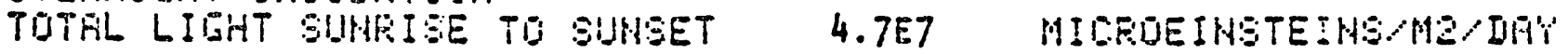

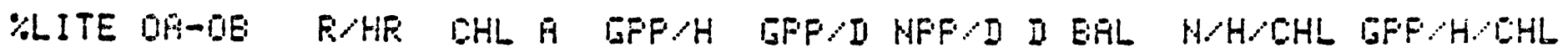

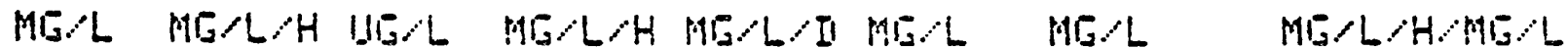

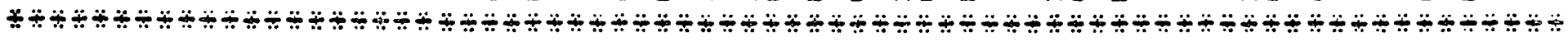

\begin{tabular}{|c|c|c|c|c|c|c|c|c|}
\hline 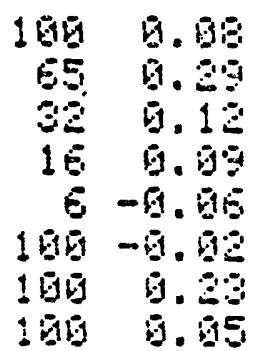 & 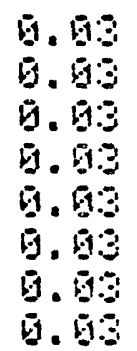 & $\begin{array}{r}5.5 \\
8.5 \\
9.6 \\
10.6 \\
9.5 \\
6.5 \\
9.6 \\
8.6\end{array}$ & 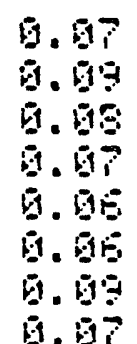 & 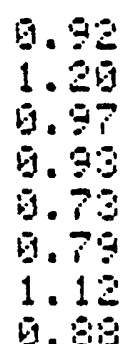 & $\begin{array}{l}0.53 \\
0.51 \\
0.50 \\
6.54 \\
0.34 \\
0.40 \\
0.73 \\
0.49\end{array}$ & 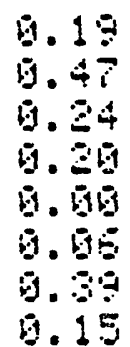 & $\begin{array}{l}5.1 \\
7.3 \\
5.8 \\
4.8 \\
5.8 \\
5.8 \\
5.3\end{array}$ & $\begin{array}{r}8.90 \\
8.95 \\
3.45\end{array}$ \\
\hline
\end{tabular}

HETTOH FOJNT

EEGIHATHE IATE BIGA1E YEAR MOHTH IATY

SURFISE 20 SUHSET 1803 IH MILITHFY HOUES

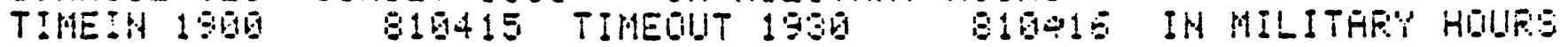

GUEFHTEHT ENEUEATIOH

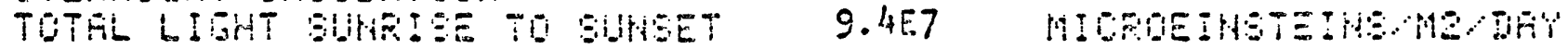

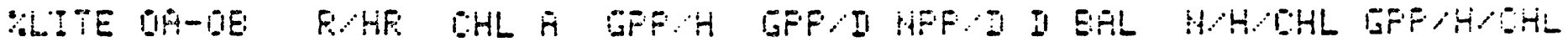

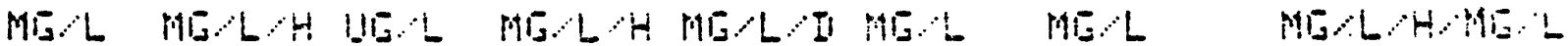

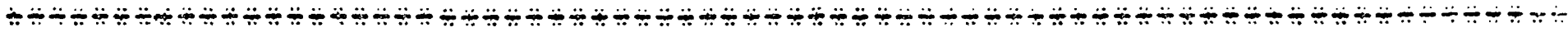

\begin{tabular}{|c|c|c|c|c|c|c|c|}
\hline 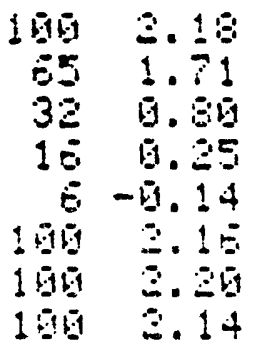 & 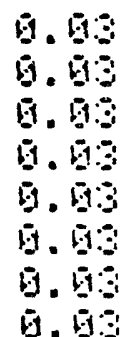 & 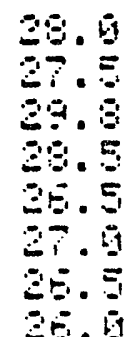 & 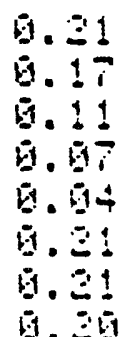 & 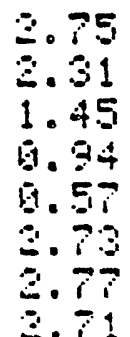 & 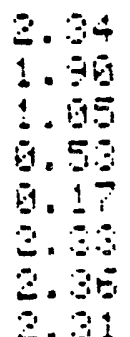 & 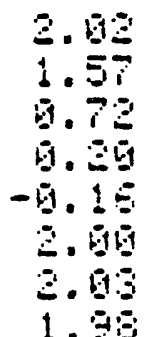 & 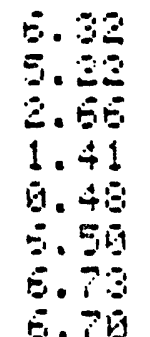 \\
\hline
\end{tabular}


HATTEN FUIHT

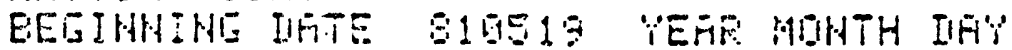

SUMPISE A4I SUHSET 1912 IH MILITAFY HOUFS

TIMEIM 2130 S19519 TIMEDUT 2315 31052 IN MILITAFI HOUES

QVEEHISHT IHLUEATIOH

TUTHL LIGHT SUHRISE TO SUHEET 8.0E7 MICROEINSTEIHS/ME/DA'

\%LITE DFI-GE FIHR CHL A RPP/H GPP/D HFF/D D EAL N/H/CHL GFP/H/CHL

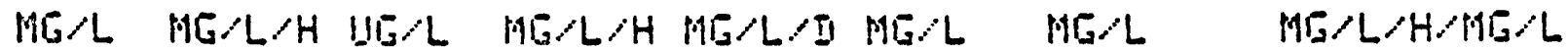

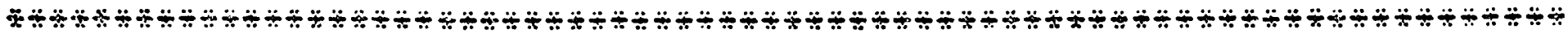

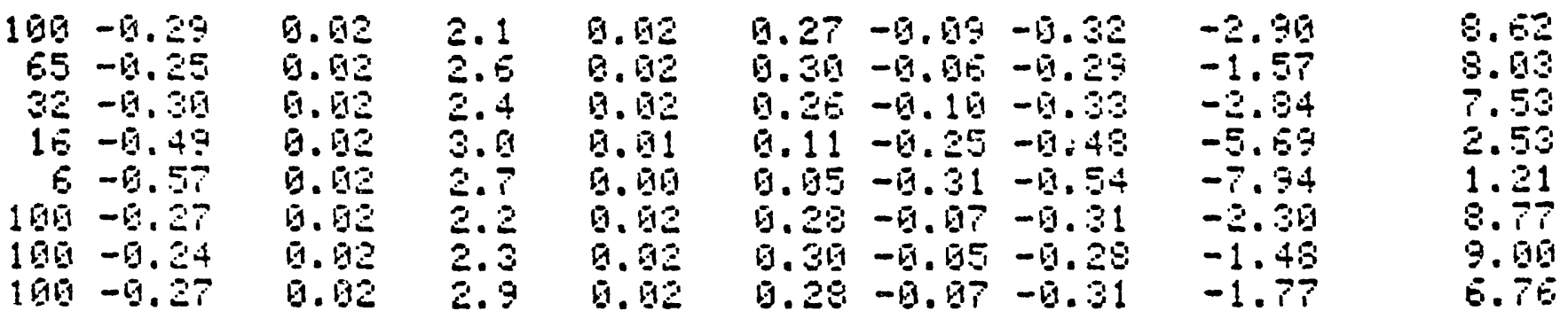

HATTOH FOIHT

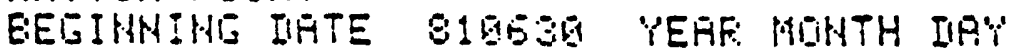

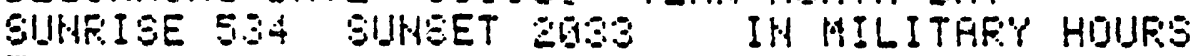

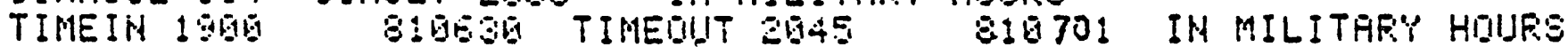

QWERHIEHT IHLIEATIOHA

TOTHL LIEHT SUHFISE TO SUHSET 8.2E7 MICRUEIHSTEIHE/MZIIAY

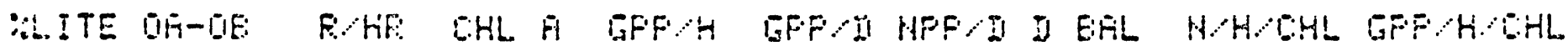

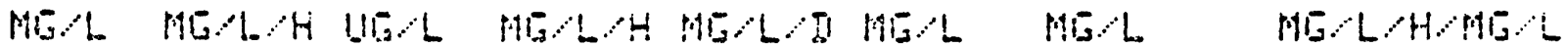

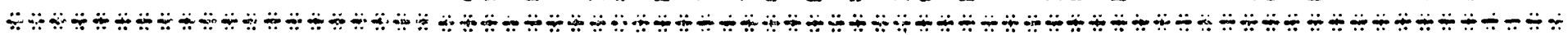

No $100-3.19$

\begin{tabular}{|c|c|c|c|c|c|c|c|}
\hline 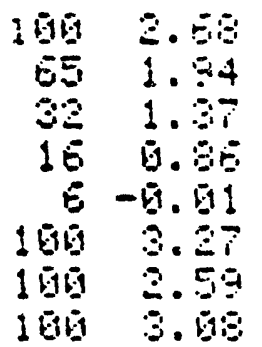 & 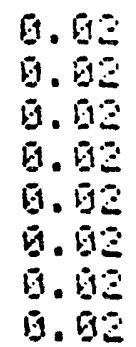 & $\begin{array}{l}36.5 \\
30.7 \\
30.5 \\
46.4 \\
36.3 \\
34.1 \\
31.7 \\
3.9\end{array}$ & $\begin{array}{l}9.21 \\
0.17 \\
0.13 \\
0.0 \% \\
0.04 \\
0.25 \\
0.31 \\
0.24\end{array}$ & $\begin{array}{l}3.21 \\
2.45 \\
1.91 \\
1.41 \\
6.55 \\
3.75 \\
3.12 \\
3.65\end{array}$ & $\begin{array}{l}2.58 \\
2.15 \\
1.58 \\
1.98 \\
0.25 \\
3.45 \\
2.79 \\
3.27\end{array}$ & $\begin{array}{l}3.58 \\
1.95 \\
1.30 \\
0.80 \\
0.92 \\
3.20 \\
2.50 \\
3.19\end{array}$ & $\begin{array}{l}6.41 \\
4.25 \\
5.85 \\
1.55 \\
9.50 \\
5.87 \\
5.85 \\
6.50\end{array}$ \\
\hline
\end{tabular}


HATTOH FOIHT

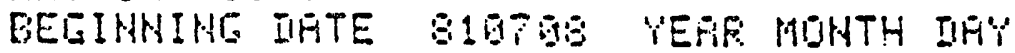

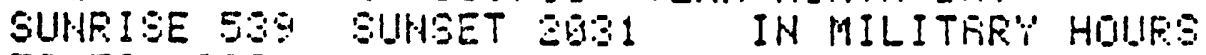

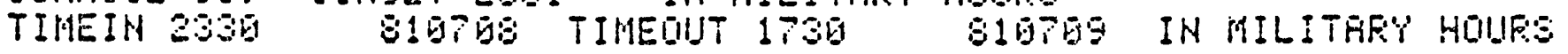

UWERHIEHT IHEUERTIOHA

TUTHL LIEHT SUHRISE TQ SUHEET M I.IE8 MICFUEIHSTEIHS/ME/IHY

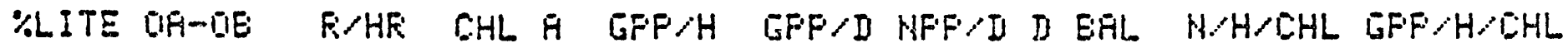

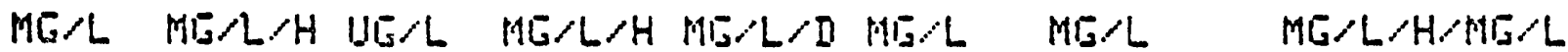

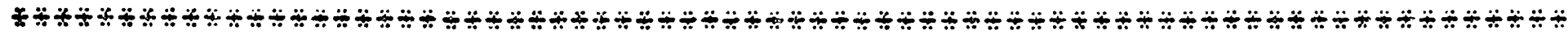

\begin{tabular}{|c|c|c|c|c|c|c|c|}
\hline $\begin{array}{l}3.48 \\
3.53 \\
3.49 \\
3.23 \\
5.51 \\
5.67 \\
3.59 \\
3.99\end{array}$ & 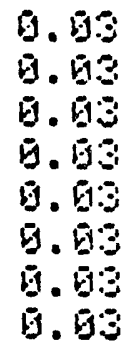 & $\begin{array}{l}25.5 \\
35.5 \\
53.5 \\
39.5 \\
40.6 \\
44.4 \\
30.3 \\
26.3\end{array}$ & $\begin{array}{l}6.34 \\
6.35 \\
6.30 \\
6.35 \\
6.59 \\
0.54 \\
0.35 \\
0.35\end{array}$ & $\begin{array}{l}5.93 \\
5.17 \\
4.93 \\
3.45 \\
1.39 \\
3.63 \\
5.27 \\
5.29\end{array}$ & 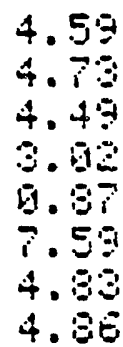 & 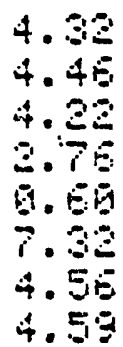 & $\begin{array}{r}12.12 \\
5.67 \\
5.65 \\
5.17 \\
1.45 \\
11.55 \\
16.78 \\
12.48\end{array}$ \\
\hline
\end{tabular}

HFTTIOHA FIIHT

EEIIHWIKG IHTE BIBT2G YEAF: MINTH IAY

SIUHFISE 54E SUHSET ZBEE IH MILITHFY HDUFE

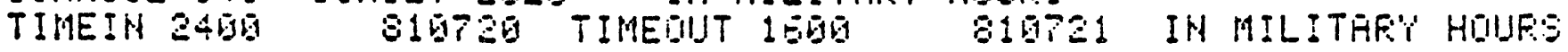

OUEFHIEHT IHELERTIDH

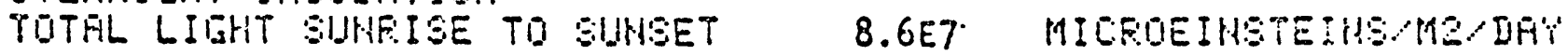

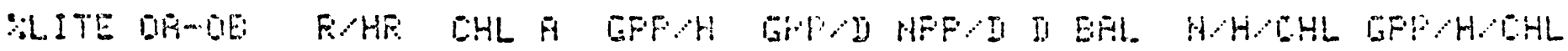

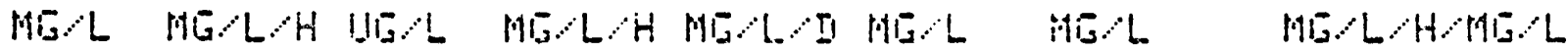

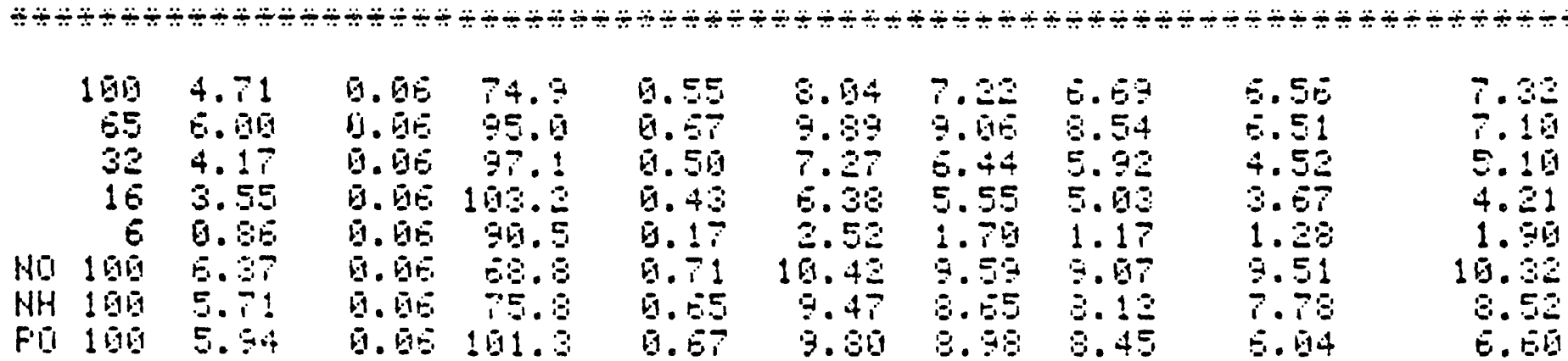


HATTOHA FIIHT

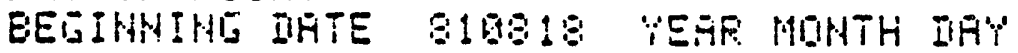

SUHFISE S14 SUHSET ISS4 IH MILITREY HOUFS

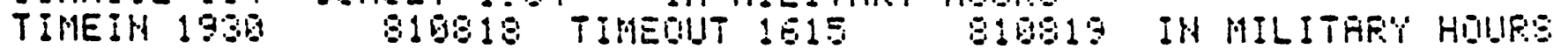

OHEFHIDHT IHEUERTIDH

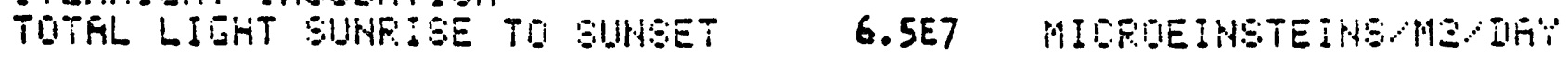

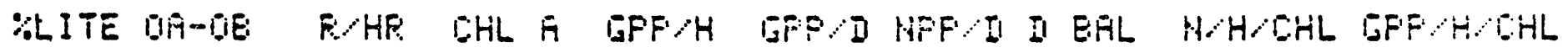

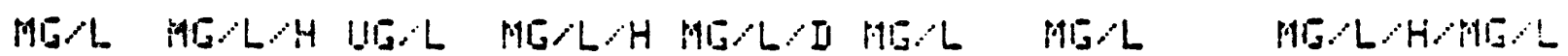

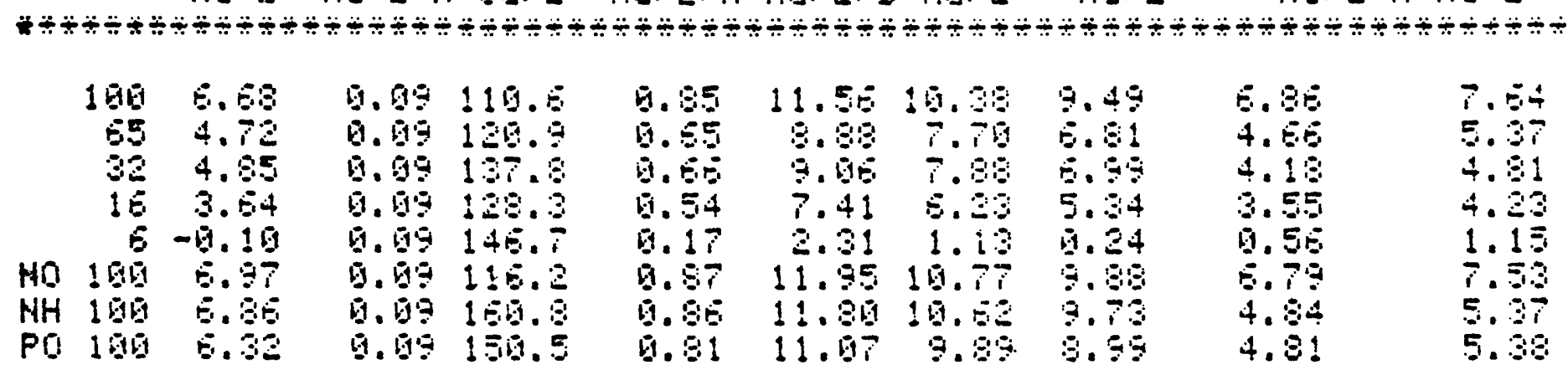


HFLLOHEHI POIHT

EEGIHHIH IHTE BGIT2S YERK MINTH DAY

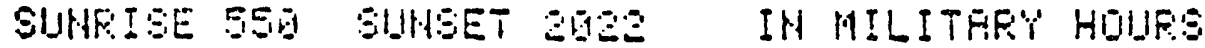

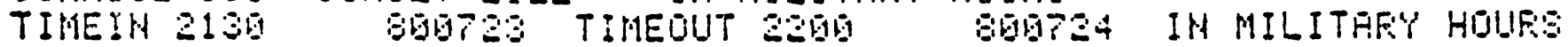

OVEFHILHT IHEUERTIOH

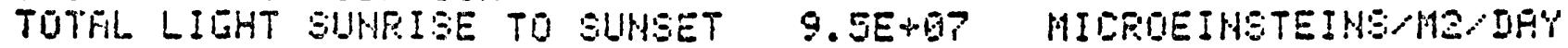

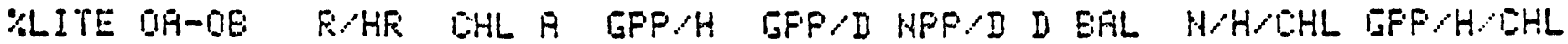

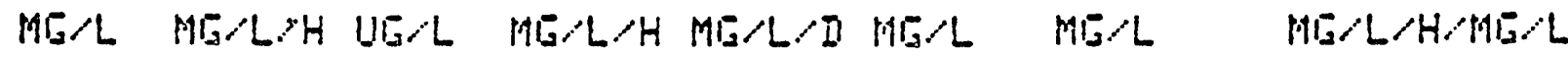

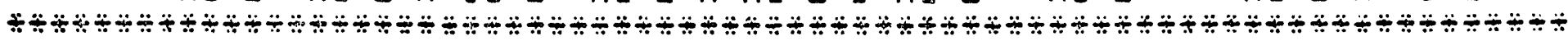

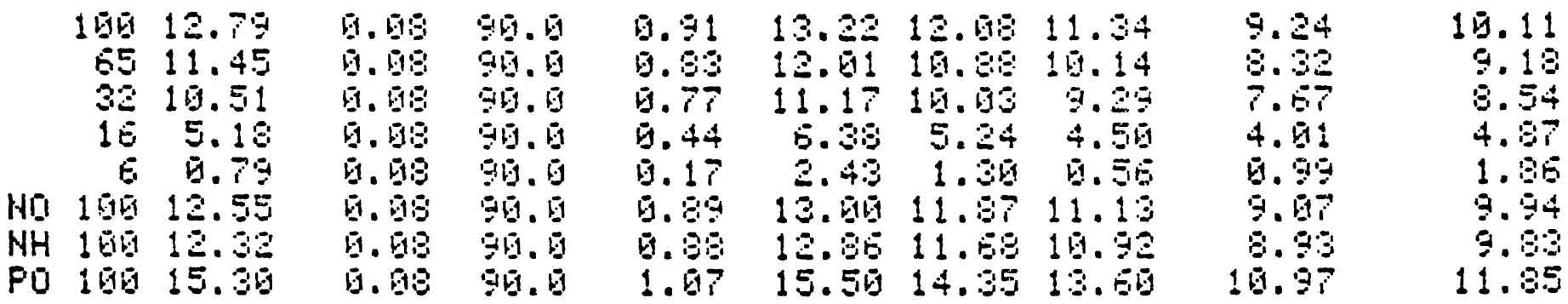

HALLOHINIE FOINT

EELIHHIHI IHTE BGGT2\% VEAF MUNTH DHY

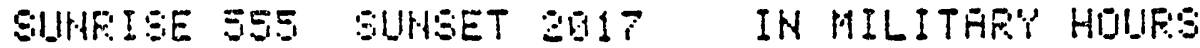

TIMETH 1730 BQ⿴囗十

QVEEHIEHT IHOUEATIDH

TOTHL LILHT SUHFISE TU SUNSET 8.3E7 MICFOEINETEIHSHO DAY

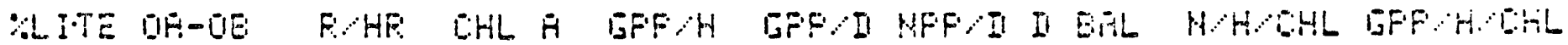

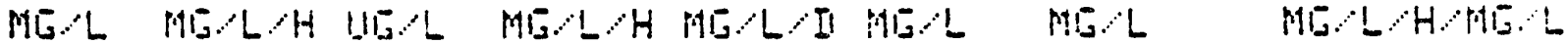

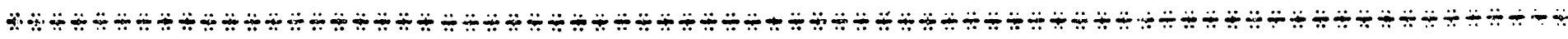

\begin{tabular}{|c|c|c|c|c|c|c|c|}
\hline $\begin{array}{r}190 \\
65 \\
32 \\
10 \\
6 \\
100 \\
160 \\
160\end{array}$ & $\begin{array}{l}9.5 \\
8.55 \\
5.35 \\
6.45 \\
5.24 \\
5.35 \\
9.94 \\
9.93\end{array}$ & 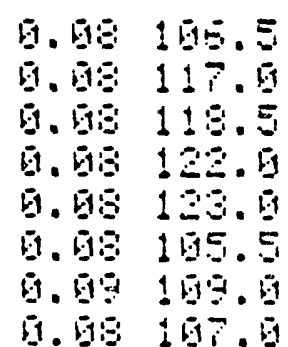 & $\begin{array}{l}1.47 \\
1.35 \\
1.80 \\
1.55 \\
6.85 \\
1.45 \\
1.45 \\
1.52\end{array}$ & 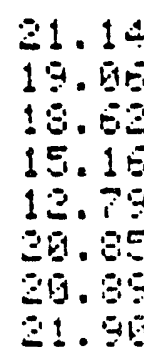 & 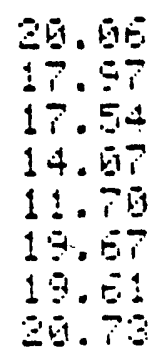 & 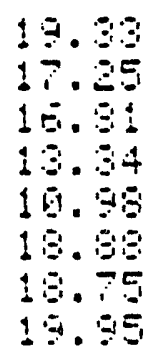 & 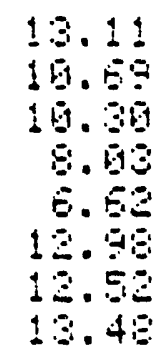 \\
\hline
\end{tabular}

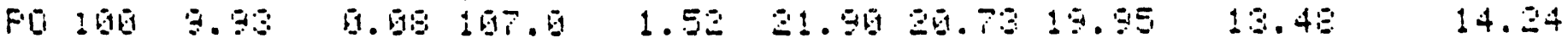


HALLOHIHI FOIHT

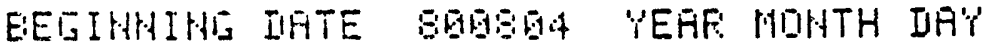

SUHEISE 559 SIIHET 2012 IN MILITARY HOUES

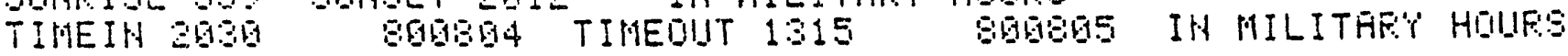

DUEFHIEHT IHLUEATIOH

TOTAL LILHT SUHAISE TO SUHSET 8.OE7 MICFOEIHSTEIHSMQYIA'Y

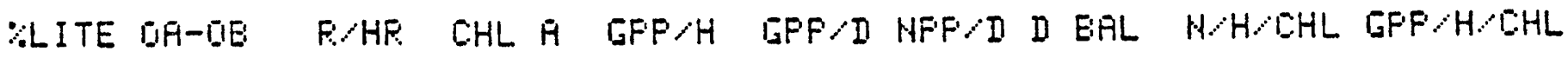

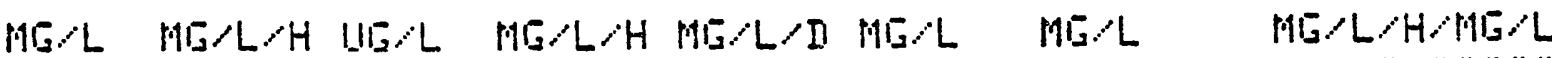

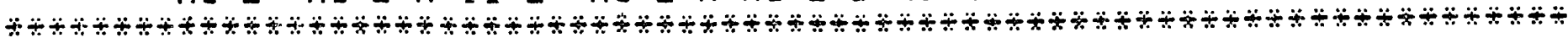

\begin{tabular}{|c|c|c|c|c|c|c|c|}
\hline $\begin{array}{l}7.43 \\
8.54 \\
7.45 \\
6.38 \\
1.58 \\
8.13 \\
7.32\end{array}$ & $\begin{array}{l}0.11 \\
0.11 \\
0.11 \\
0.11 \\
0.11 \\
0.12 \\
0.11\end{array}$ & $\begin{array}{l}84.8 \\
94.3 \\
98.8 \\
97.3 \\
85.0 \\
85.0 \\
55.3 \\
84.9\end{array}$ & $\begin{array}{l}1.27 \\
1.35 \\
1.28 \\
1.12 \\
1.45 \\
1.85 \\
1.25 \\
1.59\end{array}$ & $\begin{array}{r}18.55 \\
19.25 \\
19.18 \\
15.91 \\
6.57 \\
19.73 \\
17.39 \\
21.79\end{array}$ & $\begin{array}{r}16.53 \\
17.78 \\
16.65 \\
14.38 \\
5.05 \\
18.67 \\
16.29 \\
19.45\end{array}$ & $\begin{array}{l}15.45 \\
15.67 \\
15.85 \\
13.35 \\
3.93 \\
10.95 \\
15.25 \\
19.73\end{array}$ & $\begin{array}{r}13.71 \\
13.25 \\
11.85 \\
19.39 \\
4.18 \\
14.95 \\
13.25 \\
16.54\end{array}$ \\
\hline
\end{tabular}

HFLLOWIHI FOIHT

EELIHHIHE INTE BDGSG YEAF MOHTH IAYY

SUHAISE 604 SUHSET 2607 IH MILITARY HOURS

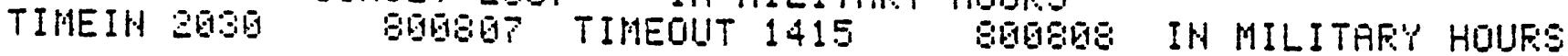

QVEFHIEHT IHEUEATIOH

TOTHL LIGHT SUHFISE TO SUHEET I.1E8 MICFOEIHETEIHSMMEIHF'

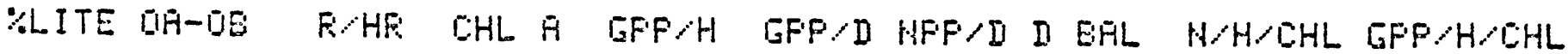

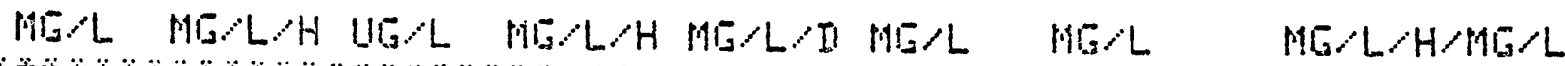

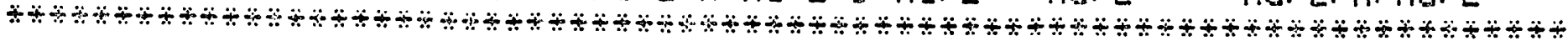

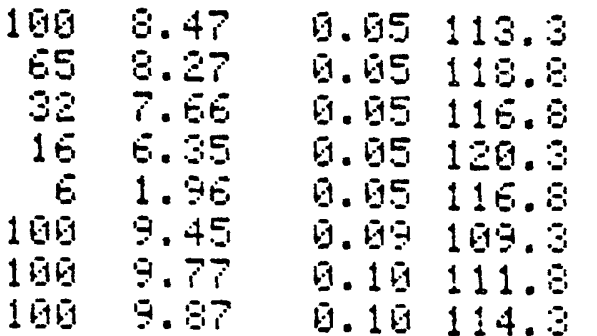

1.14

1.12

1.04

0.80

1. 34

1.36

1.42

1.42
$16.02 \quad 15.34 \quad 14.86$

15.6914 .9914 .51

14.6313 .9513 .47

12.3811 .7911 .22

$4.84 \quad 4.16 \quad 3.65$

19.6417 .7410 .82

19.9518 .4917 .45

19.9518 .5617 .58
9.64

8.99

8.56

6.92

2. 54

11.56

11.77

11.56
19.67

9.46

8.92

7.33

2.95

12.49

12. 71

12.42 
HALLOHIHE FOIHT

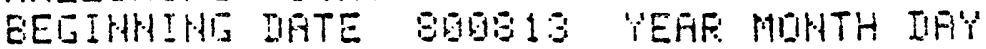

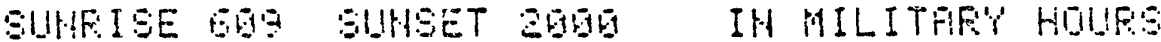

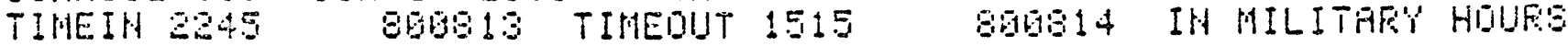

QUEEHIEHT IHIUEETTION

TOTHL LIGHT SUHAISE TO SUHSET $1.0 E 8$ MICEDEIHSTEIHSMR IAH

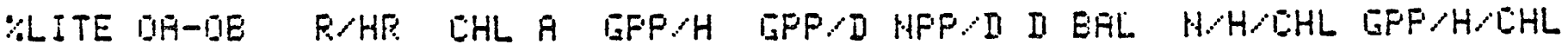

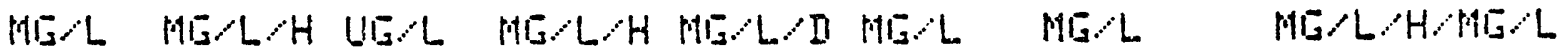

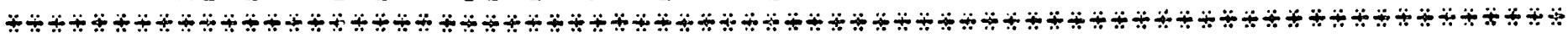

\begin{tabular}{|c|c|c|c|c|c|c|c|c|}
\hline $\begin{array}{r}106 \\
65 \\
35 \\
15 \\
8 \\
150 \\
100 \\
100\end{array}$ & $\begin{array}{r}10.60 \\
12.83 \\
9.18 \\
11.93 \\
5.40 \\
11.48 \\
11.28 \\
12.39\end{array}$ & $\begin{array}{l}0.05 \\
0.65 \\
0.05 \\
0.05 \\
0.05 \\
0.05 \\
0.11 \\
0.10\end{array}$ & $\begin{array}{l}119.0 \\
121.5 \\
124.0 \\
135.0 \\
141.5 \\
111.5 \\
111.5 \\
105.5\end{array}$ & $\begin{array}{l}1.29 \\
1.50 \\
1.12 \\
1.43 \\
9.72 \\
1.45 \\
1.43 \\
1.53\end{array}$ & $\begin{array}{r}17.94 \\
26.31 \\
15.55 \\
19.83 \\
9.92 \\
19.72 \\
19.93 \\
21.25\end{array}$ & $\begin{array}{r}15.95 \\
19.93 \\
14.98 \\
16.95 \\
9.65 \\
13.48 \\
10.35 \\
19.93\end{array}$ & $\begin{array}{r}16.32 \\
19.9 \\
14.64 \\
16.32 \\
0.41 \\
17.57 \\
17.27 \\
16.95\end{array}$ & $\begin{array}{r}11.14 \\
11.94 \\
6.55 \\
16.55 \\
4.95 \\
11.97 \\
11.97 \\
13.54\end{array}$ \\
\hline
\end{tabular}

HFILLOWIMI FOIIHT

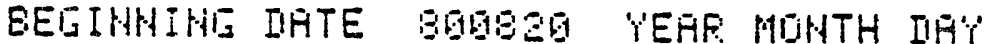

BUHEISE 514 SUHSET 1954 IH MILITARY HOUES

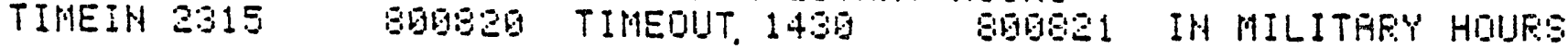

DVEFHIDHT INCUEATIOH

TOTAL LIGHT SUHRISE TO SUHSET 3.5E7 RIIEOEIHETEINEM INAT

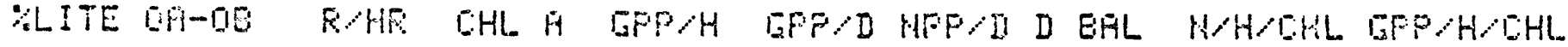

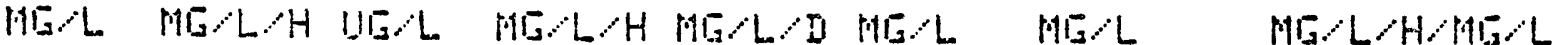

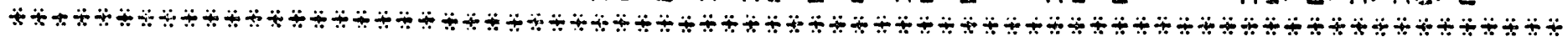

\begin{tabular}{|c|c|c|c|c|c|c|c|c|}
\hline $\begin{array}{r}196 \\
65 \\
32 \\
16 \\
6 \\
106 \\
100 \\
106\end{array}$ & $\begin{array}{l}5.29 \\
4.73 \\
3.85 \\
2.13 \\
9.12 \\
4.90 \\
5.93 \\
5.60\end{array}$ & $\begin{array}{l}0.05 \\
0.05 \\
0.05 \\
0.05 \\
0.05 \\
0.05 \\
0.05 \\
0.05\end{array}$ & $\begin{array}{l}68.9 \\
80.9 \\
6.4 \\
81.4 \\
72.4 \\
79.9 \\
78.9 \\
8.4 .4\end{array}$ & $\begin{array}{l}0.73 \\
0.57 \\
0.58 \\
0.35 \\
0.11 \\
0.89 \\
0.92 \\
0.74\end{array}$ & $\begin{array}{r}9.95 \\
9.13 \\
7.67 \\
4.83 \\
1.56 \\
9.34 \\
11.19 \\
19.13\end{array}$ & $\begin{array}{r}9.24 \\
8.42 \\
-9.96 \\
4.12 \\
9.89 \\
9.63 \\
19.42 \\
9.60\end{array}$ & $\begin{array}{l}8.71 \\
3.98 \\
9.48 \\
9.58 \\
9.10 \\
9.87 \\
9.30\end{array}$ & $\begin{array}{l}9.82 \\
7.51 \\
6.18 \\
3.79 \\
9.86 \\
8.11 \\
9.79 \\
8.79\end{array}$ \\
\hline
\end{tabular}


HALLOHINE POIHT

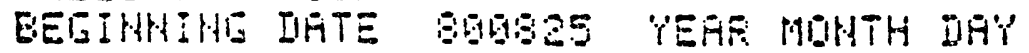

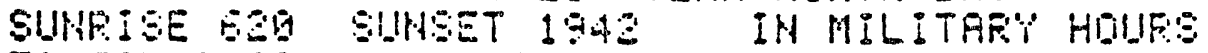

TIMEIH 24 G

QVERHIEHT IHQUEATIOH

TOTHL LIGHT SUHEYSE TO SUHSET 8.1E7 MILEOEIHSTEINSWA DHY

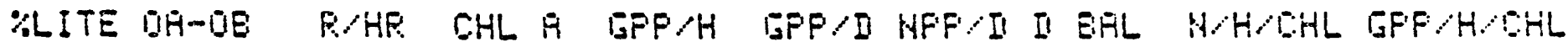

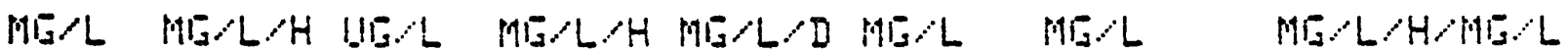

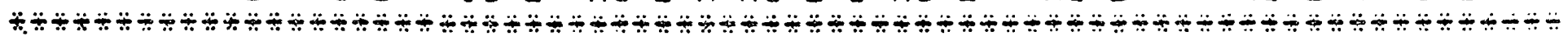

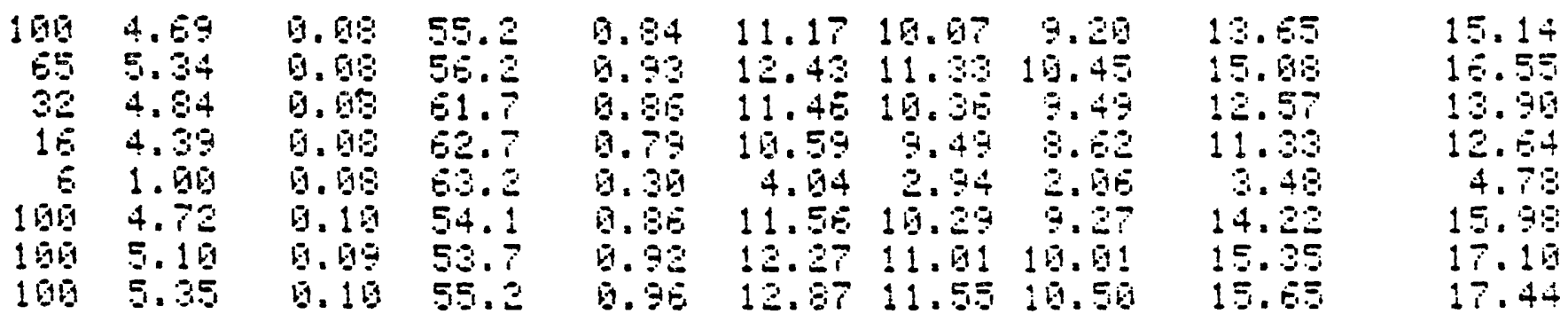

HFILLOWIHI FIIHT

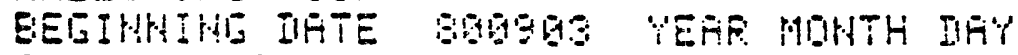

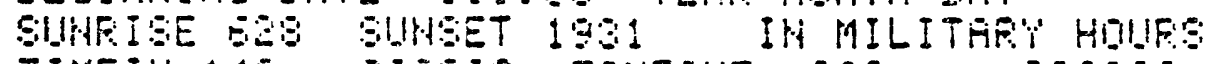

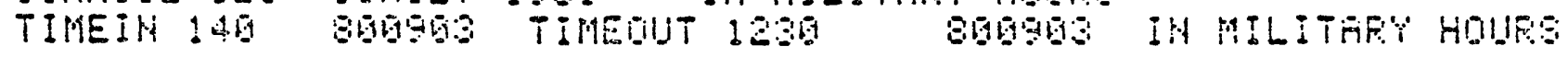

OUEAHEHT INBUEATIOH

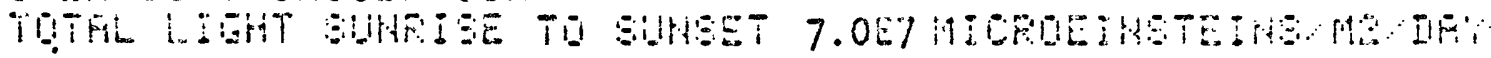

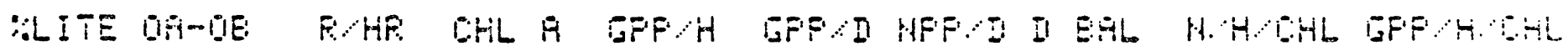

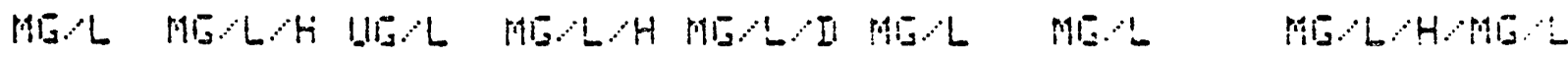

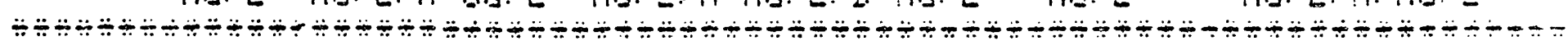

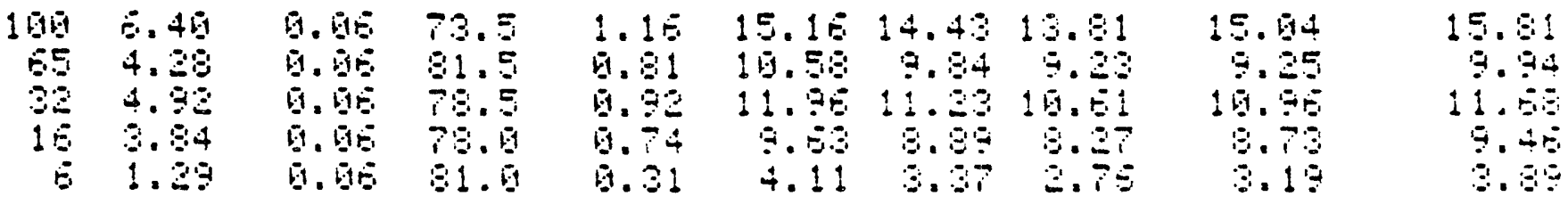


HALLOWING FIOIHT

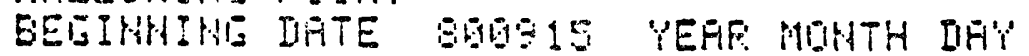

SUHFISE SAO SUNSET 191 G IH MILITAFI HOUFE

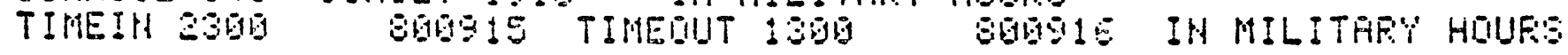

QWEEHIEHT INEUEATIOHA

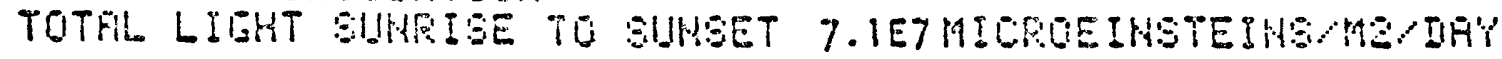

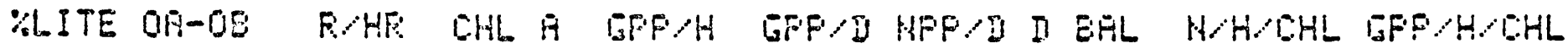

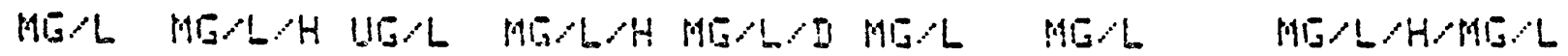

管

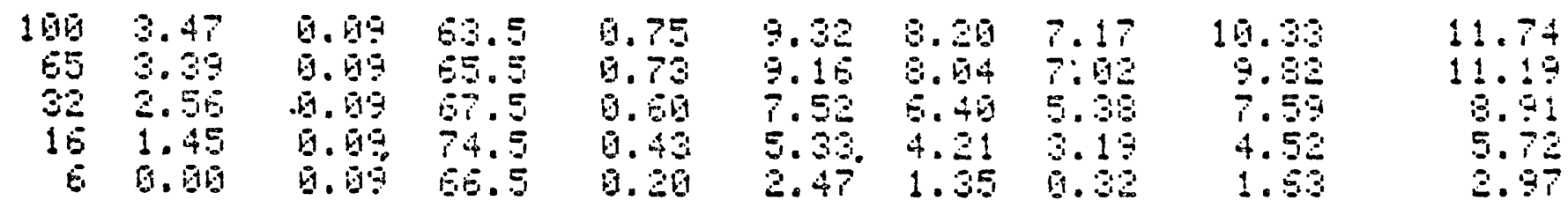

HALLOWIHE FITHT

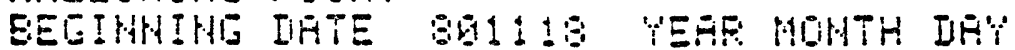

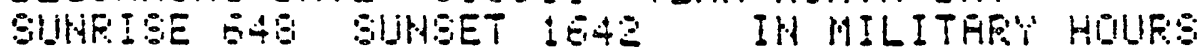

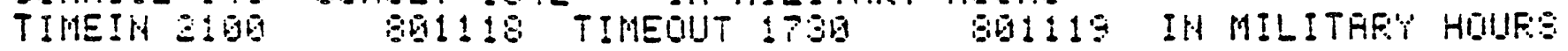

OUEFHIEHT IHEUEATTOHA

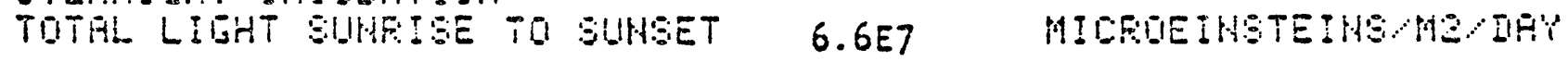

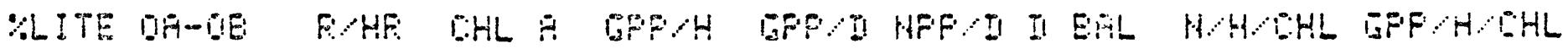

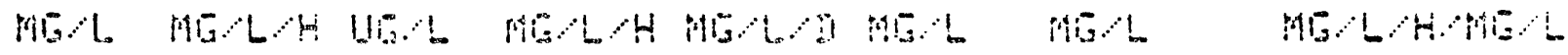

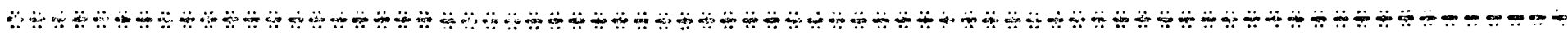

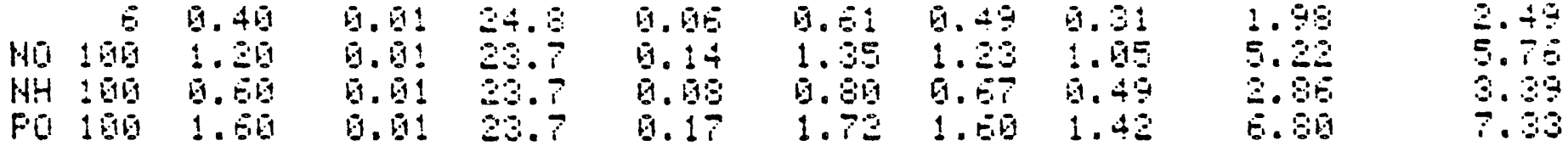


HRLLOHEHI FOIHT

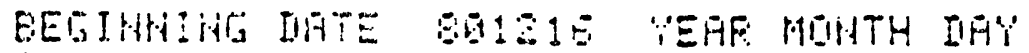

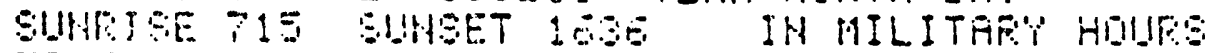

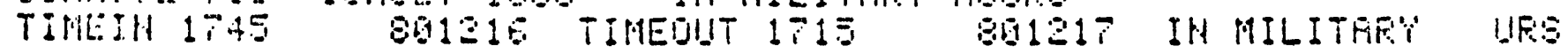

OVETHIDHT INIELERTIOH

TOTRL LIGHT BUHFISE TO SUHSET $6.9 E 7$ MICRUEIHETEIHEMMUIHT

OLITE OHADE RAHE GHL A GFFAH GFF/I MFFOD D EFL HAHCHL GFFHEHL

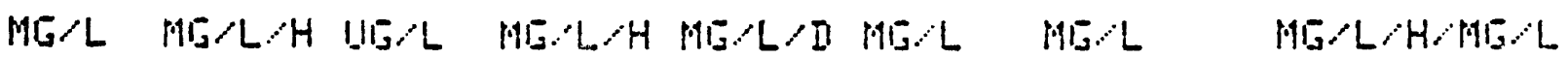

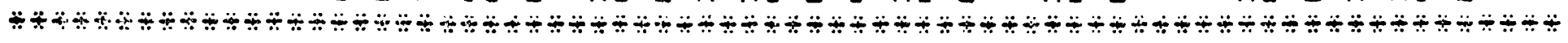

\begin{tabular}{|c|c|c|c|c|c|c|c|c|}
\hline $\begin{array}{l}1.25 \\
1.25 \\
0.35 \\
0.25 \\
9.30 \\
9.35 \\
9.25 \\
0.35\end{array}$ & 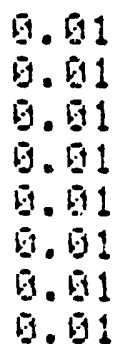 & $\begin{array}{l}15.5 \\
15.4 \\
15.5 \\
15.5 \\
17.5 \\
15.4 \\
15.5 \\
15.1\end{array}$ & 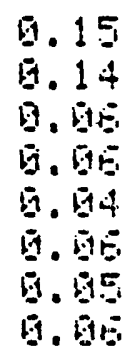 & $\begin{array}{l}1.35 \\
1.34 \\
0.54 \\
6.54 \\
6.40 \\
0.54 \\
9.45 \\
0.54\end{array}$ & $\begin{array}{l}1.25 \\
1.25 \\
9.45 \\
6.45 \\
0.31 \\
0.45 \\
6.95 \\
0.45\end{array}$ & $\begin{array}{l}1.15 \\
1.15 \\
6.21 \\
6.31 \\
0.17 \\
0.31 \\
0.01 \\
0.31\end{array}$ & $\begin{array}{l}5.85 \\
0.14 \\
2.85 \\
2.85 \\
1.85 \\
0.14 \\
-4.45\end{array}$ & $\begin{array}{l}9.47 \\
6.73 \\
3.44 \\
3.44 \\
2.44\end{array}$ \\
\hline
\end{tabular}

HALLOHIHE POIHT

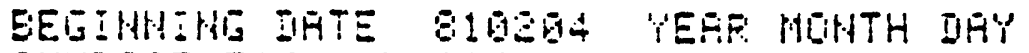

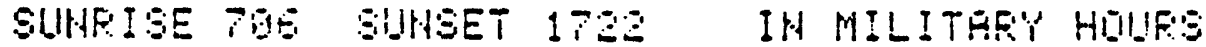

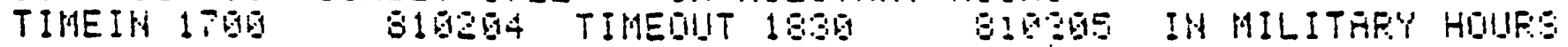

OUEFHICHT INEUEATIOHA

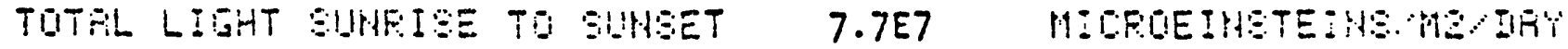

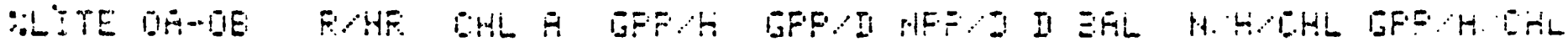

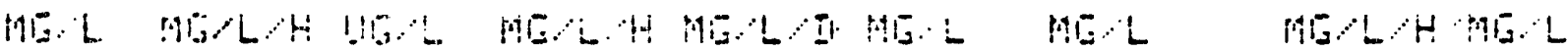

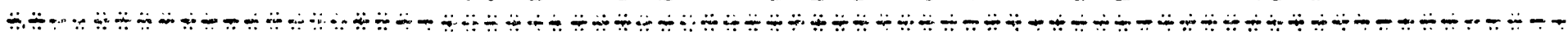

\begin{tabular}{|c|c|c|c|c|c|c|c|}
\hline 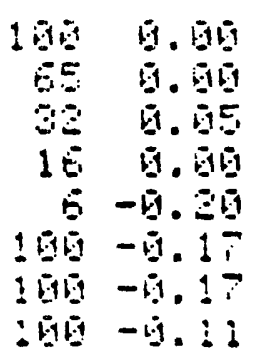 & 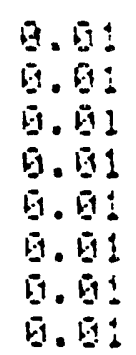 & 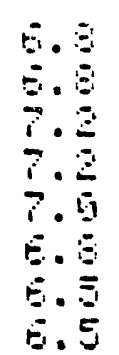 & 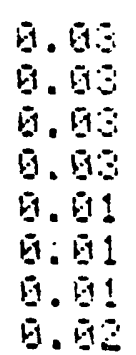 & $\begin{array}{l}0.27 \\
0.27 \\
0.25 \\
0.27 \\
0.13 \\
0.12 \\
0.17\end{array}$ & 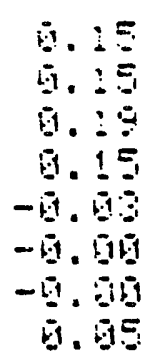 & 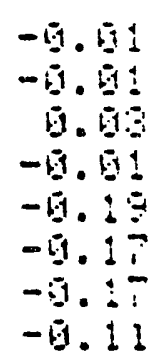 & 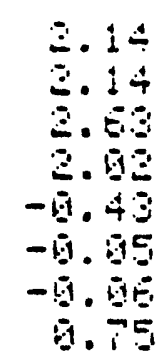 \\
\hline
\end{tabular}


HALLOUIHE POIHT

EETIHHIHE THTE S1G4G! YEFR MOHTH INAY

SUHFISE 5 SO SUHSET ISZO IH MILITARY HOURS

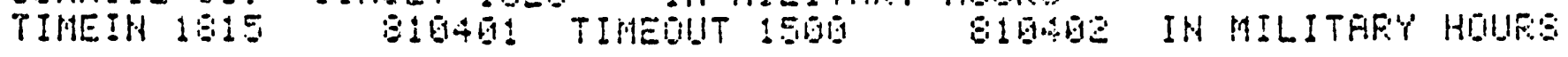

DUEFNIDHTI INLUEATIDNH

TUTHL LIGHT SUHFISE TO SUHEET 4.7E7 MICFUEIHSTEIHSME IAT

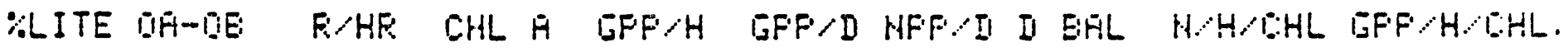

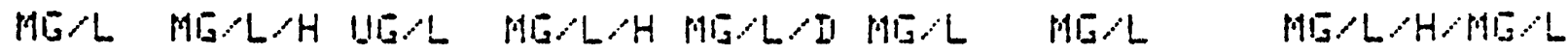

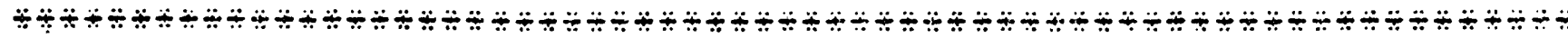

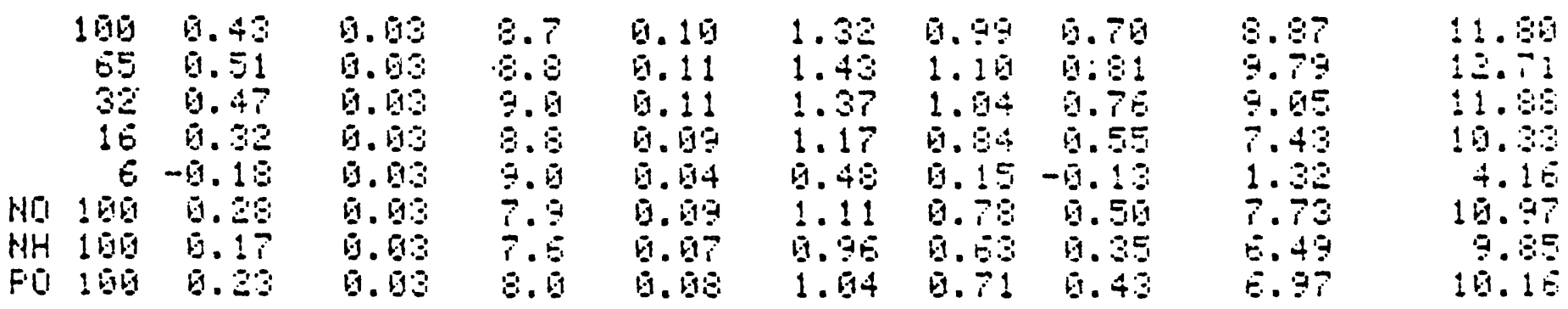

HFLLOHTHE FUIHT

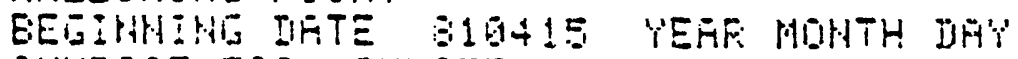

SUHFISE EOS SUHSET 1 SOS IH MILITHEI HIUES

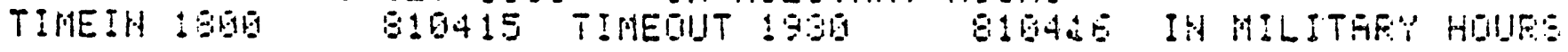

DUEFHIEHT IHE!EATIOH

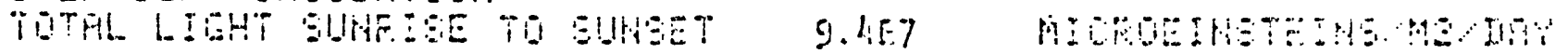

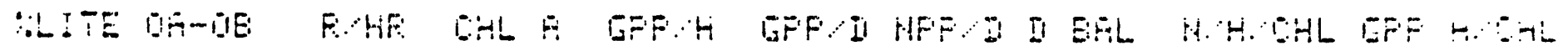

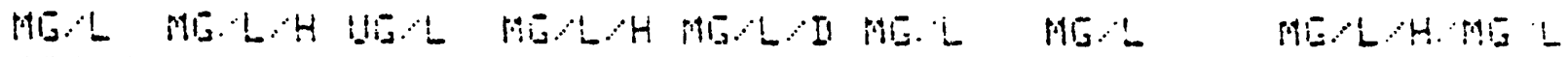

\begin{tabular}{|c|c|c|c|c|c|c|c|c|}
\hline 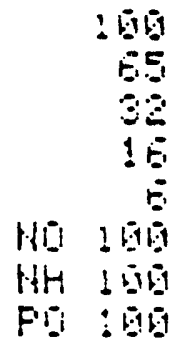 & 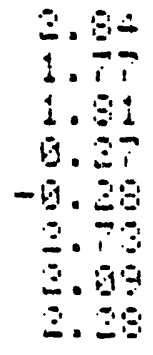 & 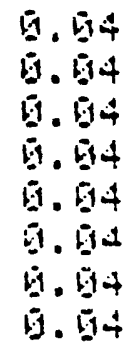 & 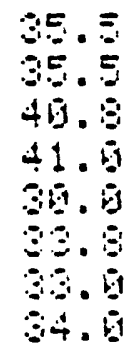 & 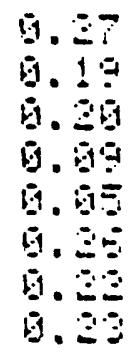 & 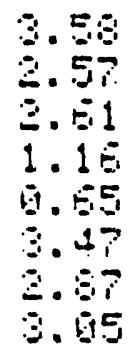 & 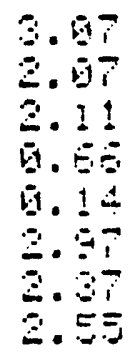 & 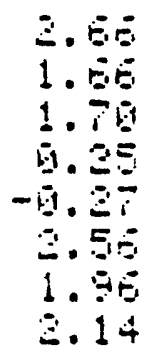 & $\begin{array}{l}5.55 \\
4.45 \\
3.85 \\
1.35 \\
0.85 \\
6.50 \\
5.40 \\
5.50\end{array}$ \\
\hline
\end{tabular}


HALLOWIHE FUIHT

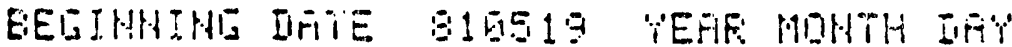

SUHPISE 441 SIHEET 1912 IH MILITARY HOLFS

TIMEIM EZS

QWEEHITHT INCUEATIOH

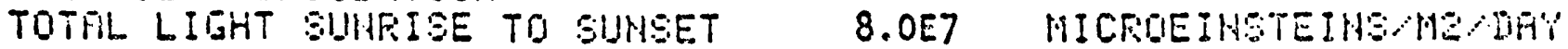

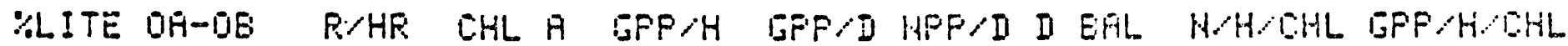

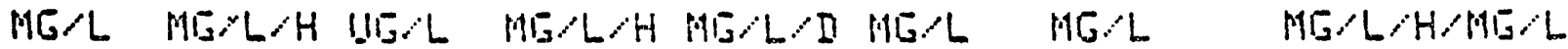

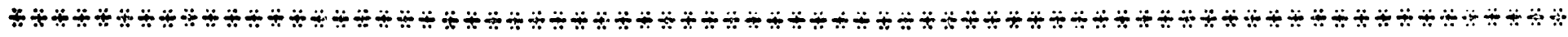

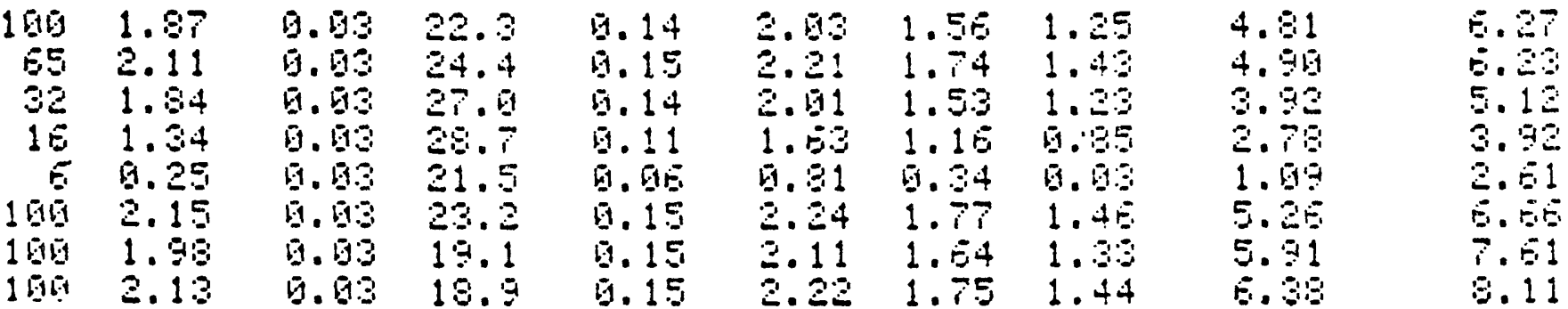

HFLLOUIHE FOIHT

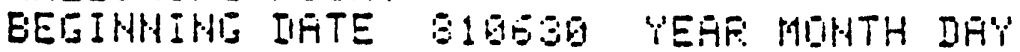

SUIHFISE 5O4 SUNSET ZESS IH MILITAFI' HDURS

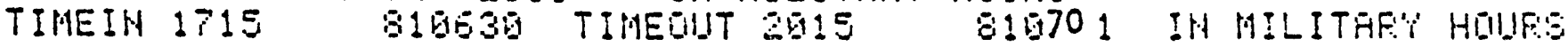

QWEFHIEHT IHEUEATIOH

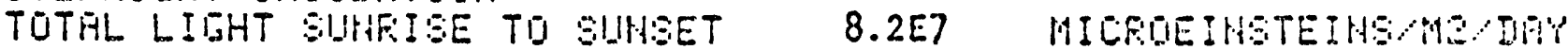

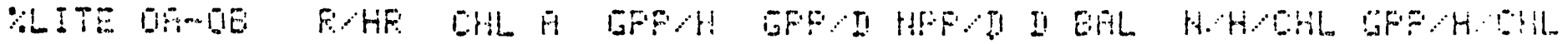

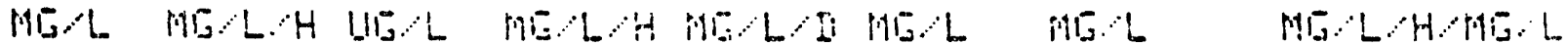

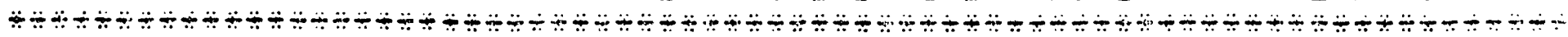

\begin{tabular}{|c|c|c|c|c|c|c|c|c|}
\hline $\begin{array}{l}5.45 \\
3.56 \\
0.45 \\
1.75 \\
6.15 \\
4.35 \\
4.96 \\
4.51\end{array}$ & 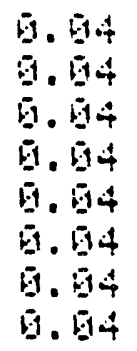 & 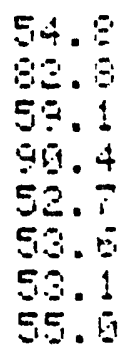 & 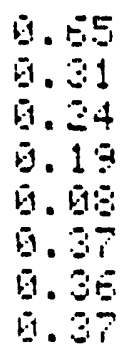 & $\begin{array}{l}9.77 \\
4.57 \\
3.56 \\
3.54 \\
1.25 \\
5.54 \\
5.34 \\
5.56\end{array}$ & $\begin{array}{l}5.17 \\
4.57 \\
3.50 \\
2.24 \\
6.54 \\
4.94 \\
4.79 \\
4.56\end{array}$ & 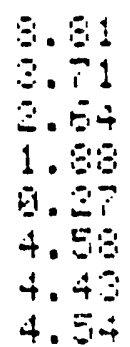 & $\begin{array}{r}11.15 \\
3.25 \\
3.35 \\
1.55 \\
6.50 \\
5.15 \\
5.05\end{array}$ & 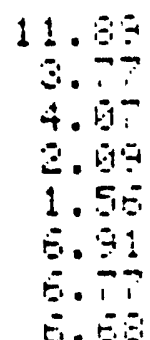 \\
\hline
\end{tabular}


HALLOHUHE FOIHT

EEGIMHIHG TIATE SIGTHS TEAF MOHTH DAY

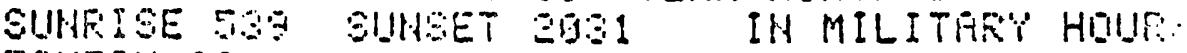

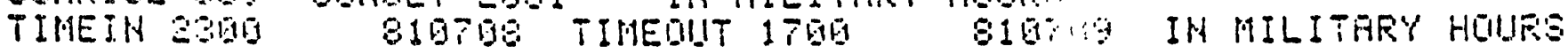

OMEFHITHT IHCHERTIDH

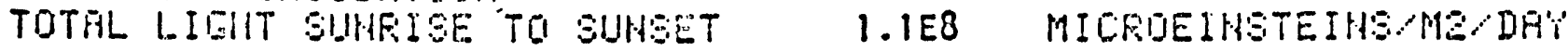

\%LITE DATDE R

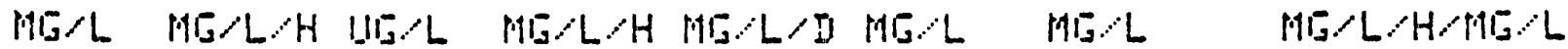

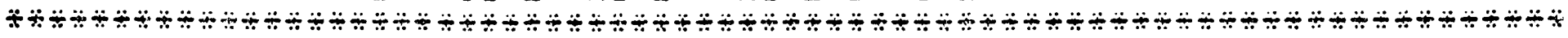

\begin{tabular}{|c|c|c|c|c|c|c|c|c|c|}
\hline $\begin{array}{r}196 \\
65 \\
32 \\
16 \\
6 \\
190 \\
106\end{array}$ & $\begin{array}{l}5.49 \\
5.73 \\
5.42 \\
4.65 \\
2.35 \\
6.35 \\
6.34 \\
6.73\end{array}$ & 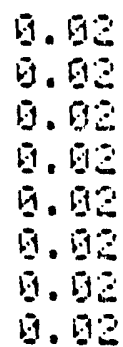 & $\begin{array}{l}51.3 \\
59.4 \\
55.1 \\
52.6 \\
52.6 \\
45.6 \\
51.5 \\
57.9\end{array}$ & $\begin{array}{l}4.59 \\
0.53 \\
0.56 \\
0.43 \\
0.23 \\
9.55 \\
0.55 \\
0.53\end{array}$ & 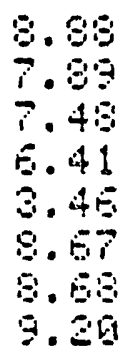 & $\begin{array}{l}5.54 \\
7.55 \\
7.34 \\
5.17 \\
3.25 \\
0.43 \\
6.44 \\
8.95\end{array}$ & 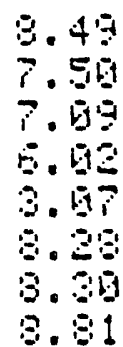 & $\begin{array}{r}11.32 \\
7.41 \\
7.35 \\
7.95 \\
4.15 \\
12.30 \\
11.92 \\
10.46\end{array}$ & $\begin{array}{r}11 \cdot 54 \\
7 \cdot 54 \\
7 \cdot 61 \\
3 \cdot 29 \\
4 \cdot 47 \\
12 \cdot 5\end{array}$ \\
\hline
\end{tabular}

HALLOWIHI FOIHT

EEGIHHIHL IATE SIGTZE TEAR MOHTH IAT

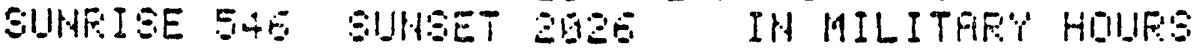

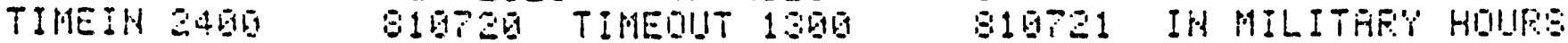

GUEFHIGHT IHEUEATIOH

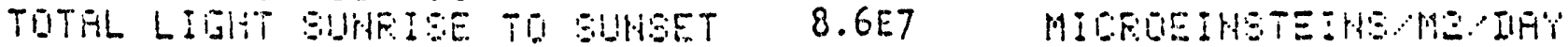

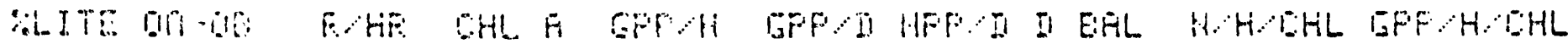

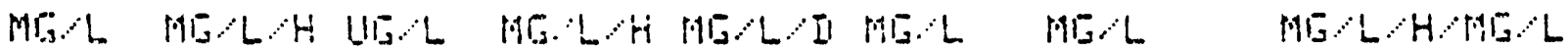

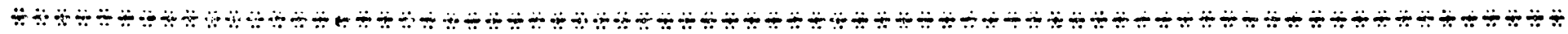

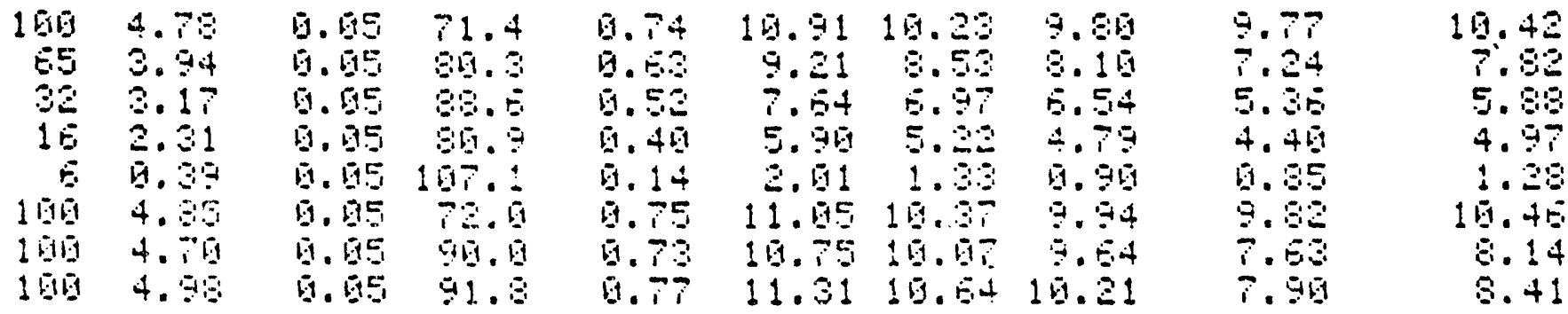




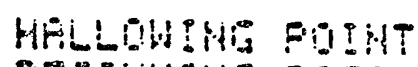

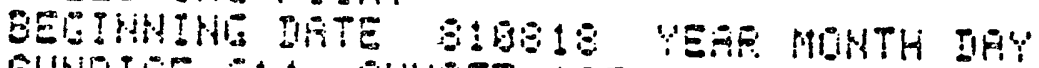

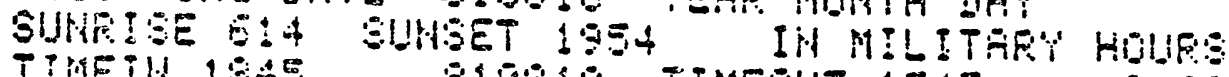

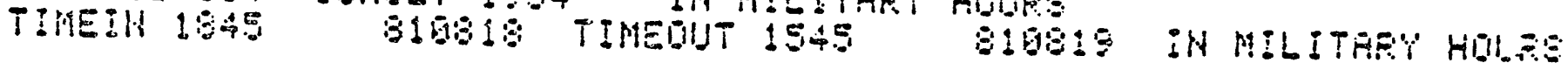

QUEFHISHE IHE!UERTIDH

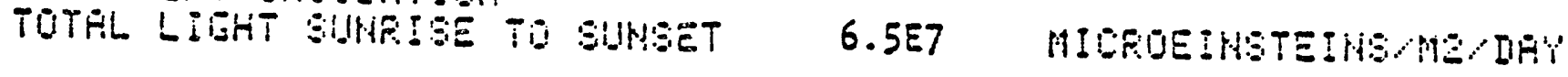

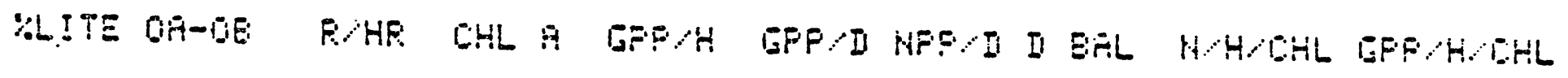

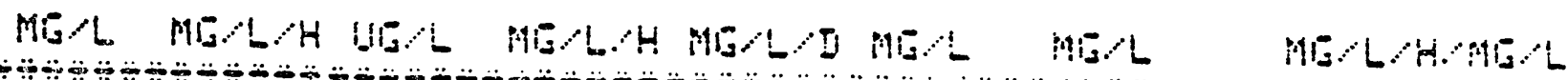

\begin{tabular}{|c|c|c|c|c|c|c|c|c|c|}
\hline 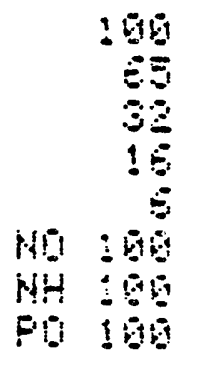 & 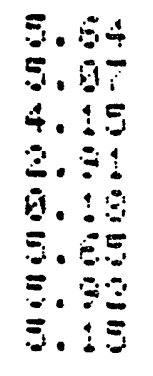 & $\begin{array}{l}0.67 \\
0.07 \\
0.97 \\
0.97 \\
0.07\end{array}$ & 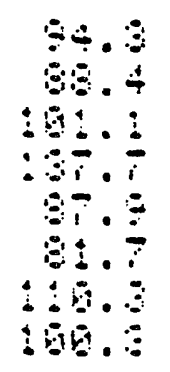 & 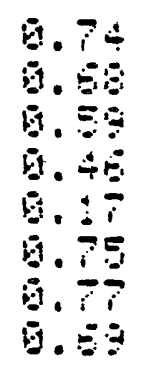 & 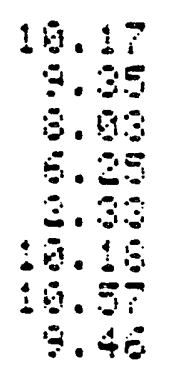 & 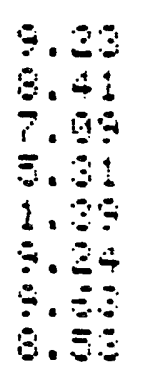 & 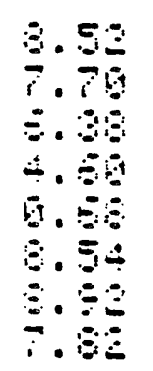 & 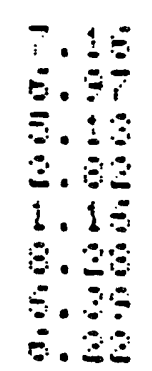 & 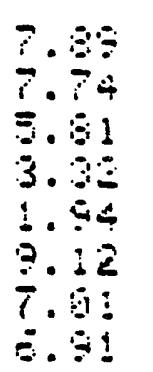 \\
\hline
\end{tabular}


IOHIELRE FOIHT

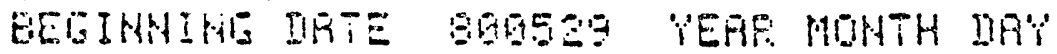

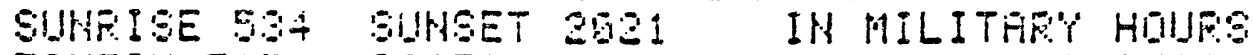

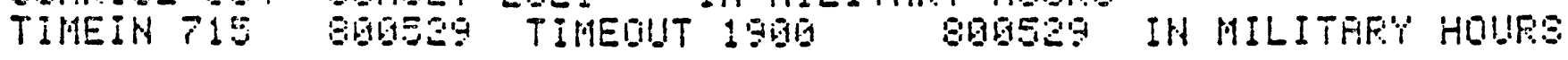

INAY INEUERTIOH DHLY

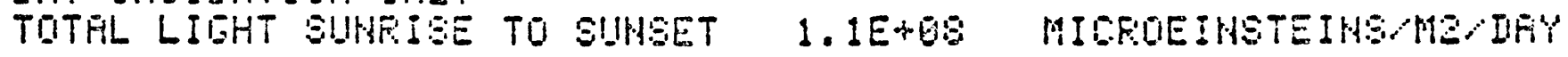

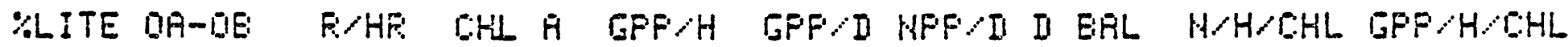

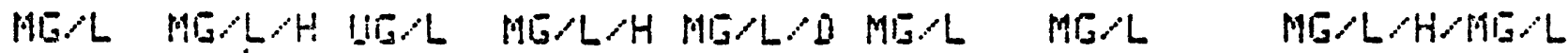

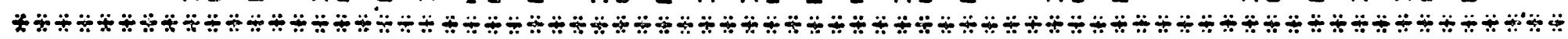

\begin{tabular}{|c|c|c|c|c|c|c|c|c|}
\hline 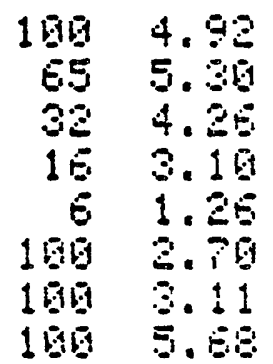 & 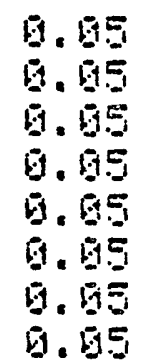 & $\begin{array}{l}49.5 \\
49.5 \\
49.5 \\
49.5 \\
49.5 \\
49.5 \\
49.5 \\
49=\end{array}$ & 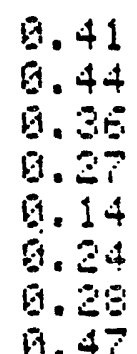 & $\begin{array}{l}5.63 \\
5.45 \\
5.815 \\
4.63 \\
2.96 \\
3.50 \\
4.12 \\
6.96\end{array}$ & 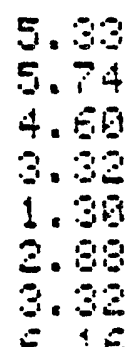 & 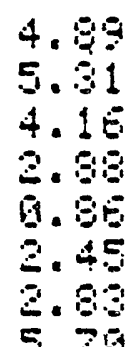 & $\begin{array}{l}7.25 \\
i .35 \\
5.25 \\
4.54 \\
1.77 \\
3.94 \\
4.54\end{array}$ & $\begin{array}{l}8.24 \\
8.81 \\
7.25 \\
5.56 \\
2.74 \\
4.89 \\
5.52\end{array}$ \\
\hline
\end{tabular}

IOULLAS FUIHT

BEGIHAIHL IATE BDGEZS YEAP MONTH IAY

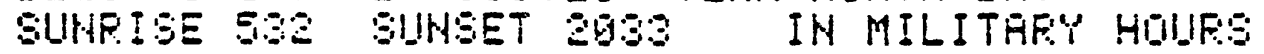

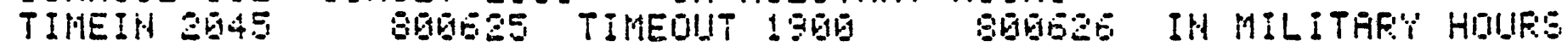

QUEFHIEHT IMEIERTIOH

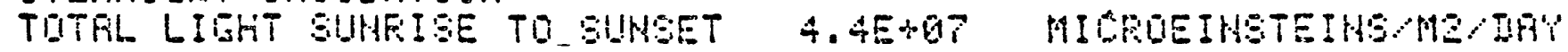

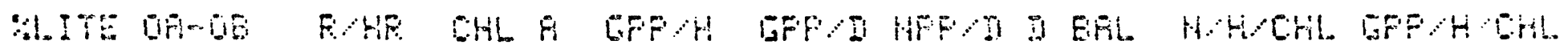

MEL MELH WEL MELH MEL IJ MEL MEL MELHALL

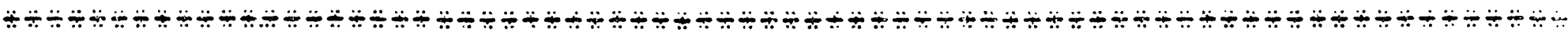

\begin{tabular}{|c|c|c|c|c|c|c|c|c|}
\hline 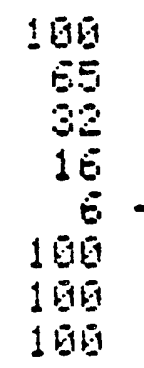 & $\begin{array}{r}3.30 \\
3.14 \\
1.80 \\
1.25 \\
-9.20 \\
3.13 \\
3.60 \\
3.65\end{array}$ & $\begin{array}{l}9.65 \\
0.65 \\
0.95 \\
0.95 \\
0.65 \\
0.65\end{array}$ & $\begin{array}{l}31.4 \\
31.4 \\
31.4 \\
31.4 \\
31.4 \\
31.4 \\
31.4 \\
31.4\end{array}$ & $\begin{array}{l}0.25 \\
0.30 \\
0.31 \\
0.15 \\
0.31 \\
0.35 \\
0.36\end{array}$ & $\begin{array}{l}3.94 \\
4.81 \\
3.15 \\
2.75 \\
1.60 \\
4.65 \\
5.21 \\
4.55\end{array}$ & 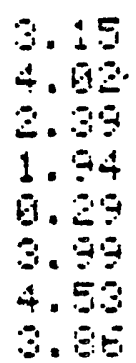 & $\begin{array}{r}2.67 \\
3.54 \\
1.92 \\
1.47 \\
-19.19 \\
3.59 \\
4.12 \\
3.45\end{array}$ & 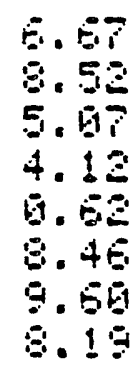 \\
\hline
\end{tabular}


Qunitisers

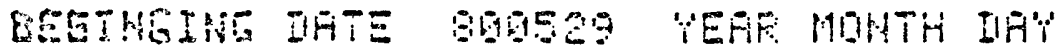

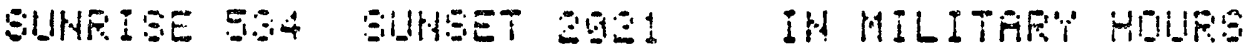

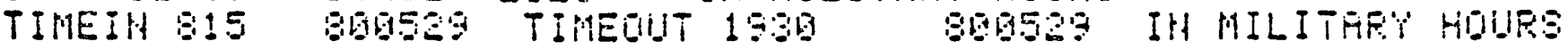

MAY IMEUEATIDH BHLY

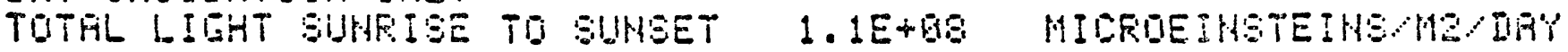

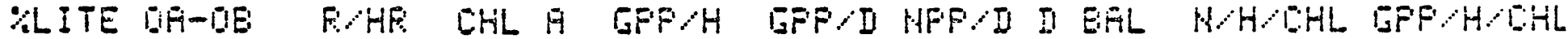

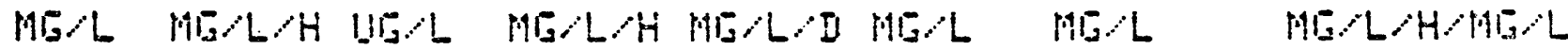

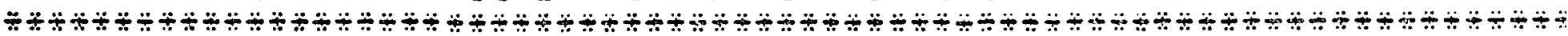

\begin{tabular}{|c|c|c|c|c|c|c|c|}
\hline $\begin{array}{l}5.13 \\
5.53 \\
4.45 \\
2.39 \\
1.915 \\
5.97\end{array}$ & 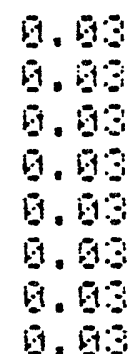 & $\begin{array}{l}24.3 \\
-4.3 \\
-4.3 \\
24.3 \\
-4.3 \\
24.3 \\
24.3\end{array}$ & $\begin{array}{l}6.45 \\
0.42 \\
6.35 \\
6.20 \\
9.15 \\
6.45 \\
6.24\end{array}$ & 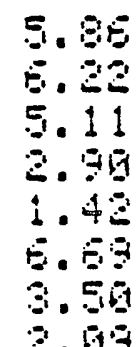 & $\begin{array}{l}5.41 \\
5.79 \\
4.57 \\
0.45 \\
6.97 \\
6.95 \\
3.95 \\
1.64\end{array}$ & 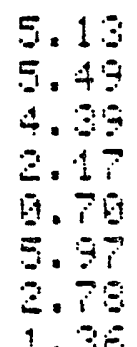 & 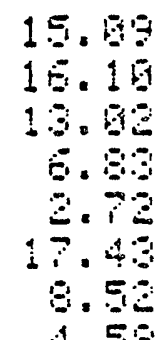 \\
\hline
\end{tabular}

Quithitico

BEGINNING IATE BEGESE YEAR MONTH IAY

SUHFISE $5 \mathrm{~A}$ SUNSET 2 OS IH MILITHEY HOUES

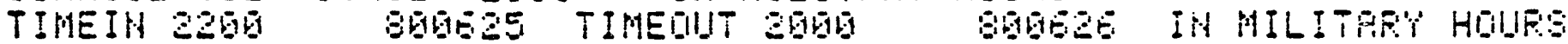

OUEFHIEHT INOUEATIOH

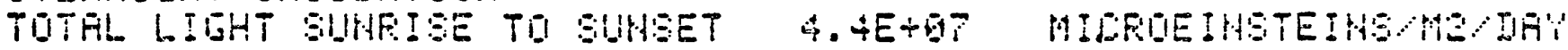

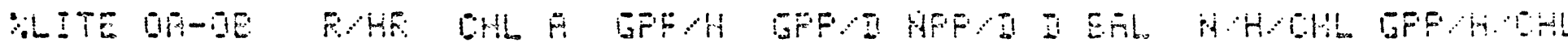

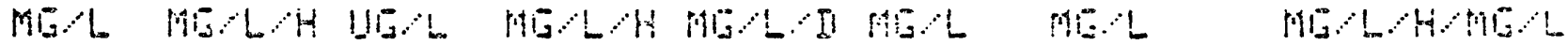

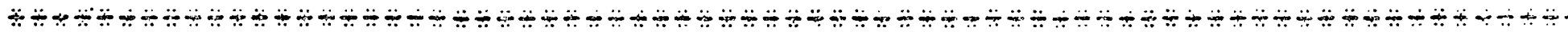

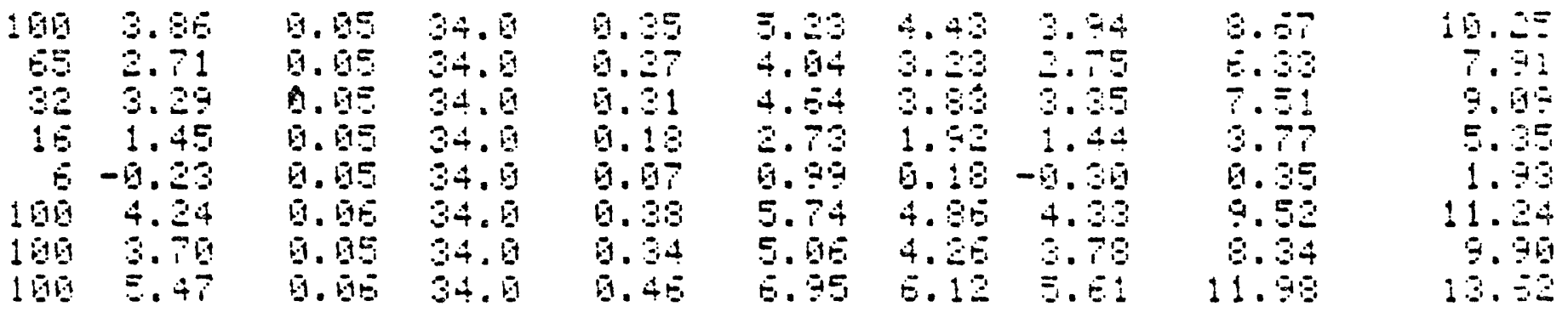


QUFHTSE:

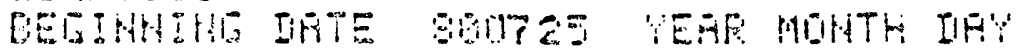

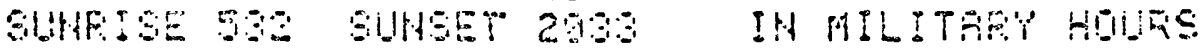

TIMEIH ZOG

QWEFHILHT INEUEATIDH

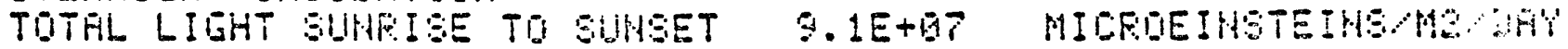

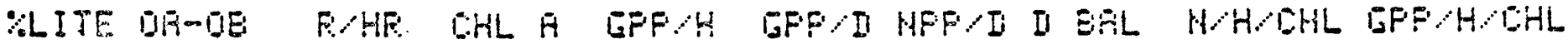

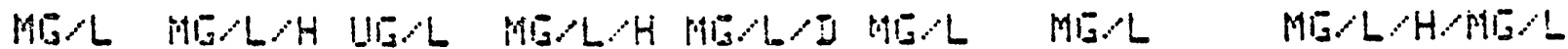

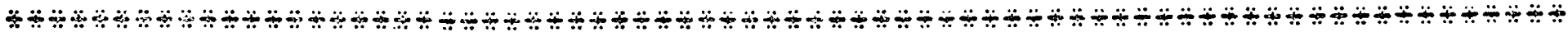

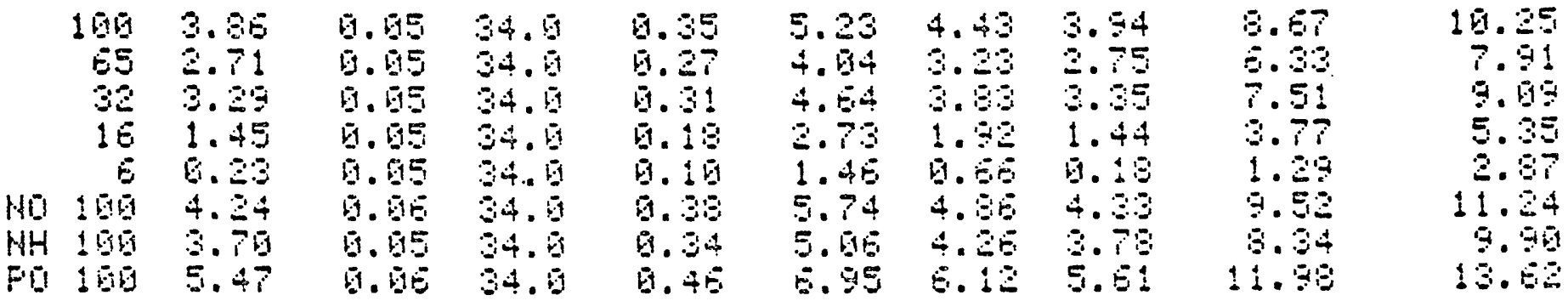

\section{DOUGLS PDiTT}

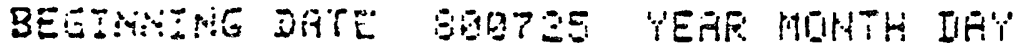

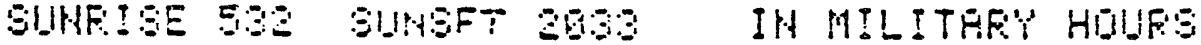

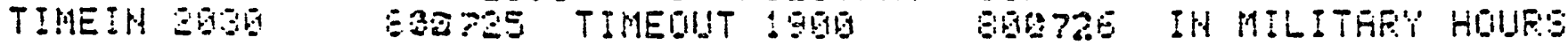

QUERHIEHT IHEUEATIH

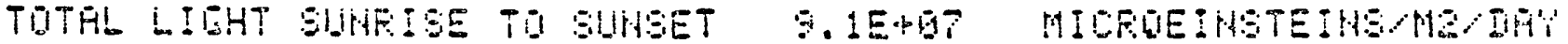

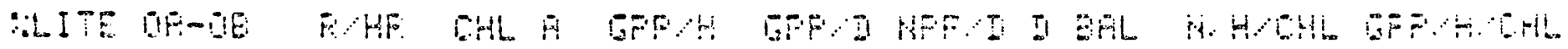

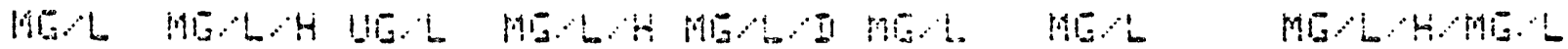

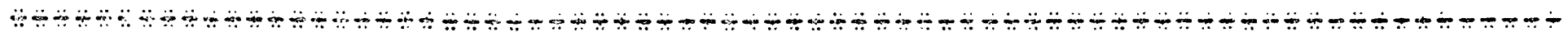

\begin{tabular}{|c|c|c|c|c|c|c|c|c|}
\hline 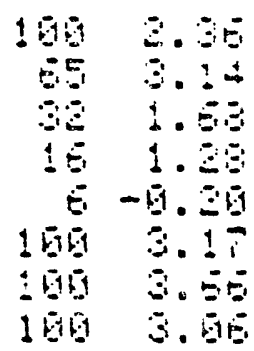 & 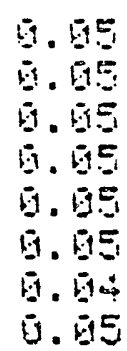 & $\begin{array}{l}3.4 \\
31.4 \\
3.4 \\
31.4 \\
31.4 \\
31.4 \\
31.4 \\
31.4 \\
31.4\end{array}$ & 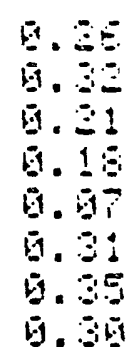 & $\begin{array}{l}3.94 \\
4.85 \\
3.15 \\
3.75 \\
1.68 \\
4.58 \\
5.21 \\
4.55\end{array}$ & 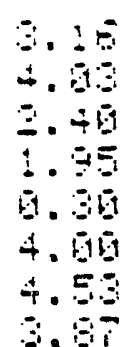 & $\begin{array}{r}2.50 \\
3.50 \\
1.95 \\
1.40 \\
-4.15 \\
3.55 \\
4.15 \\
3.40\end{array}$ & 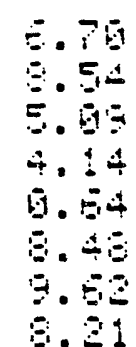 & 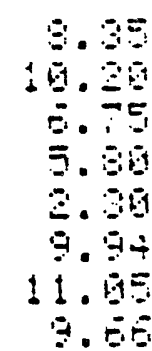 \\
\hline
\end{tabular}


RUAHTIEO

EETIMNIMI DATE SQI11S YEAF MONTH DAT

SIUNRISE GAS SIINEET ISHC IN MILITAR'Y HDIURS

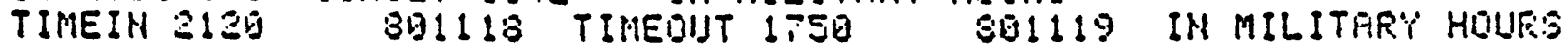

OVERMILHT INCIEATIINN

TOTHL LIGHT SUHRISE TO SUHEET

MICROEIHSTEIHE/ME/DAY

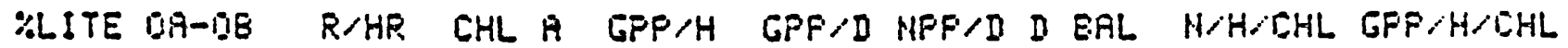

MG/L MG/L/H UR/L MIS/L/H MG/L/D MIS/L MIJ/L MIS/L/H/MG/L

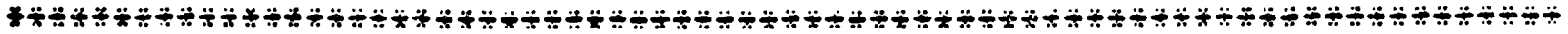

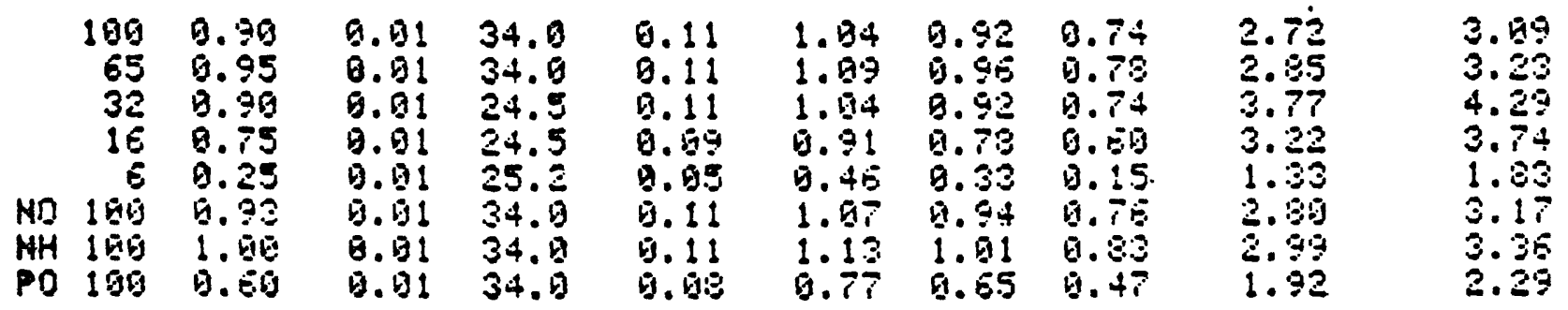

QUIRTTIED

BERIHAINI DATE SQISIS YERR MONTH DAY

SUHRISE 715 SLHEET IE.3E IN MILITAFY' HOURS

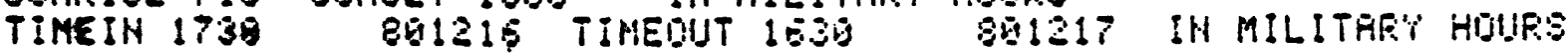

OWEFHIEHT IHCUEAT IDH

TOTHL LIBHT EUNKISE TO SIINSET

MICRDEIHSTEINSN DAY

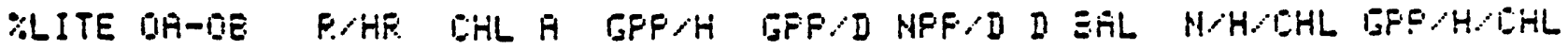

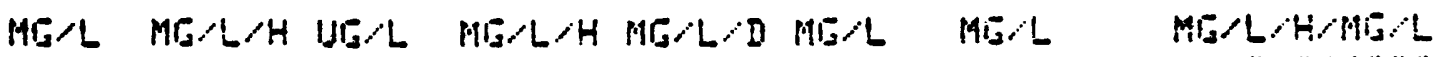

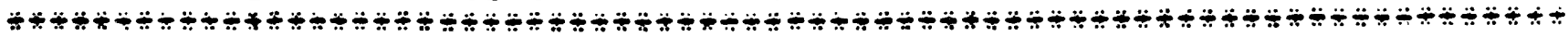

\begin{tabular}{|c|c|c|c|c|c|c|c|}
\hline $\begin{array}{r}195 \\
65 \\
32 \\
15 \\
5 \\
109 \\
160 \\
156\end{array}$ & $\begin{array}{l}9.75 \\
9.55 \\
0.55 \\
0.55 \\
9.45 \\
0.75 \\
0.85 \\
0.5\end{array}$ & $\begin{array}{l}0.91 \\
9.91 \\
0.91 \\
0.91 \\
0.11 \\
0.11 \\
0.1\end{array}$ & $\begin{array}{l}25.5 \\
25.5 \\
37.5 \\
37.3 \\
27.5 \\
35.6 \\
35.5 \\
56.5\end{array}$ & 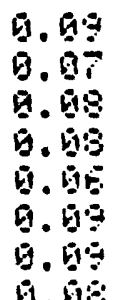 & 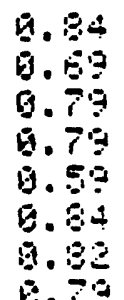 & $\begin{array}{l}1.79 \\
0.63 \\
6.74 \\
0.74 \\
9.53 \\
0.79 \\
0.79 \\
9.74\end{array}$ & $\begin{array}{l}0.75 \\
9.55 \\
0.55 \\
9.55 \\
9.45 \\
8.75 \\
0.85 \\
0.85\end{array}$ \\
\hline
\end{tabular}

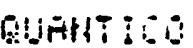

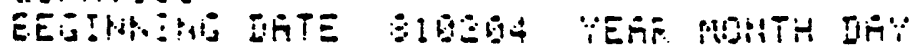

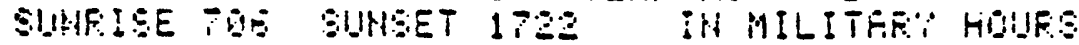

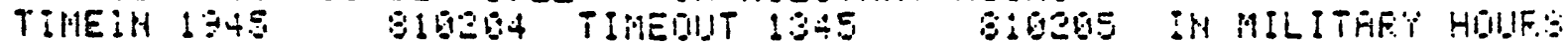

QWEFHISTT IMLUERTIOH

TITHL LIEHT BUHFI'SE TO BUHSET

MICROEIHETEINEMZIITI

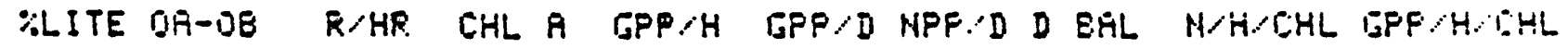

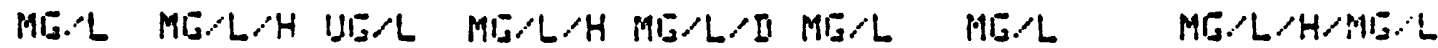

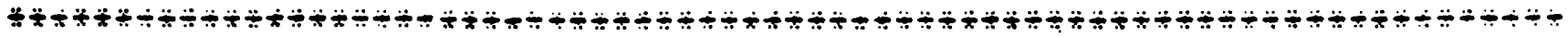

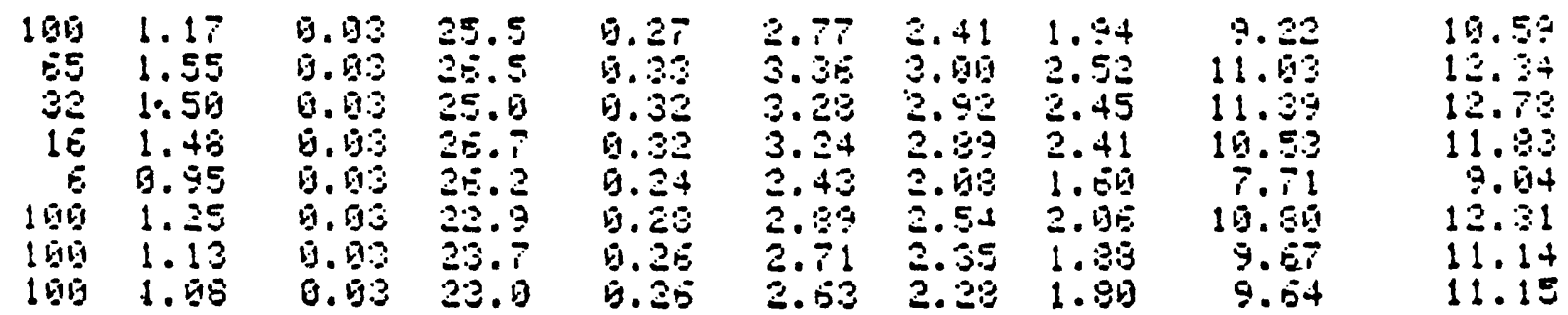




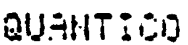

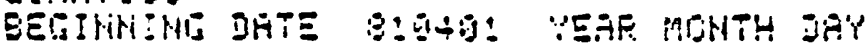

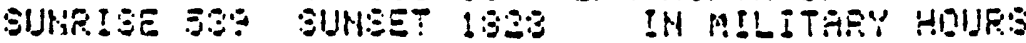

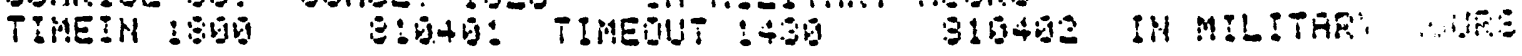

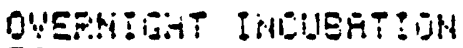

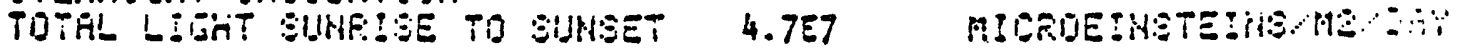

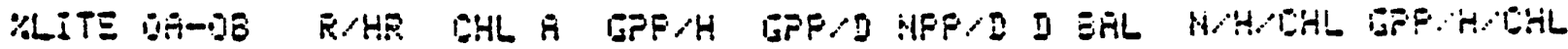

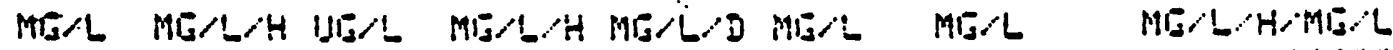

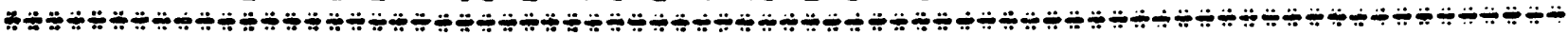

\begin{tabular}{|c|c|c|c|c|c|c|c|}
\hline $\begin{array}{rr}1019 & 9.23 \\
53 & 0.43 \\
32 & 0.35 \\
15 & 0.33 \\
5 & 0.33 \\
1010 & 0.35 \\
105 & 0.33 \\
100 & 0.31\end{array}$ & $\begin{array}{l}0.92 \\
0.02 \\
0.02 \\
0.02 \\
0.02 \\
6.02 \\
9.02 \\
0.92\end{array}$ & $\begin{array}{l}7.2 \\
7.3 \\
3.3 \\
5.3 \\
5.3 \\
7.3 \\
7.5 \\
7.4\end{array}$ & $\begin{array}{l}9.95 \\
0.19 \\
0.19 \\
0.05 \\
0.018 \\
0.15 \\
0.15 \\
0.11\end{array}$ & $\begin{array}{l}1.55 \\
1.35 \\
1.23 \\
1.15 \\
1.50 \\
1.35 \\
1.25 \\
1.45\end{array}$ & $\begin{array}{l}0.74 \\
1.04 \\
0.92 \\
0.87 \\
0.74 \\
0.35 \\
0.95 \\
1.15\end{array}$ & 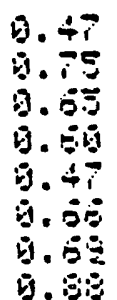 & 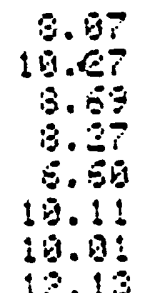 \\
\hline
\end{tabular}

DUFHTIBO

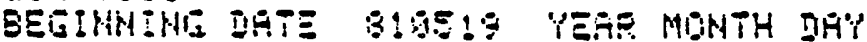

SLINRISE $4+1$ SIJNEET ISIZ IM M!LITHFY HDUR:

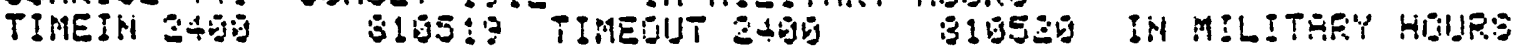

QYEFHIEHT INEUEATIIN

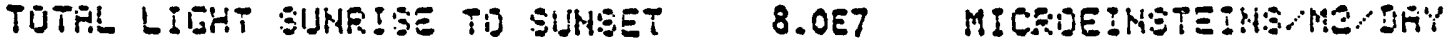

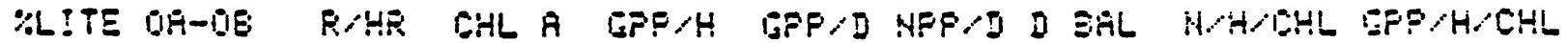

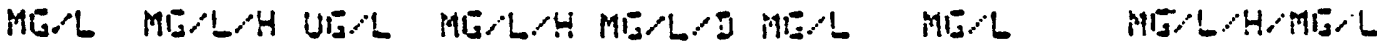
前

\begin{tabular}{|c|c|c|c|c|c|c|c|c|}
\hline $\begin{array}{r}105 \\
65 \\
32 \\
15 \\
5 \\
100 \\
100\end{array}$ & $\begin{array}{l}5.25 \\
5.67 \\
5.34 \\
4.48 \\
2.01 \\
3.35 \\
5.10 \\
5.43\end{array}$ & 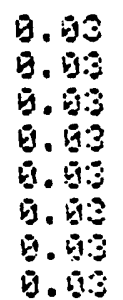 & $\begin{array}{l}75.5 \\
73.7 \\
72.5 \\
83.3 \\
67.7 \\
73.1 \\
73.0 \\
6\end{array}$ & $\begin{array}{l}0.31 \\
9.3: 3 \\
6.32 \\
0.37 \\
6.14 \\
0.32 \\
0.31\end{array}$ & $\begin{array}{l}4.57 \\
4.3 ! \\
4.55 \\
3.35 \\
3.67 \\
4.59 \\
4.45 \\
4.69\end{array}$ & $\begin{array}{l}4.13 \\
4.37 \\
4.15 \\
3.44 \\
\vdots .58 \\
4.14 \\
4.65\end{array}$ & $\begin{array}{l}3.84 \\
4.55 \\
3.37 \\
3.15 \\
1.34 \\
5.85 \\
3.71 \\
3.94\end{array}$ & $\begin{array}{l}3.72 \\
3.73 \\
3.94 \\
3.34 \\
1.23 \\
3.95 \\
3.83 \\
0.93\end{array}$ \\
\hline
\end{tabular}

Gliniti:

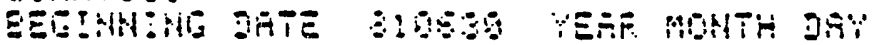

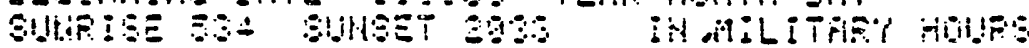

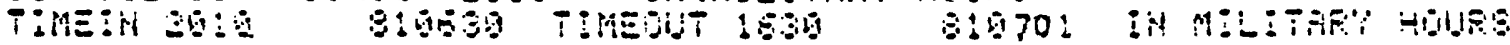

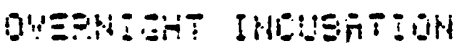

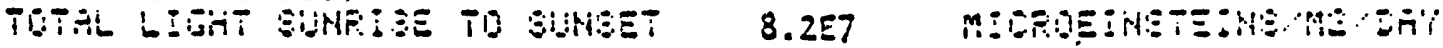

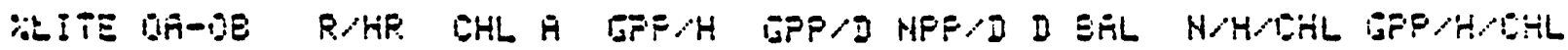

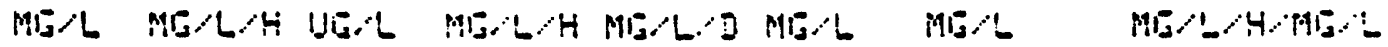
ئزي

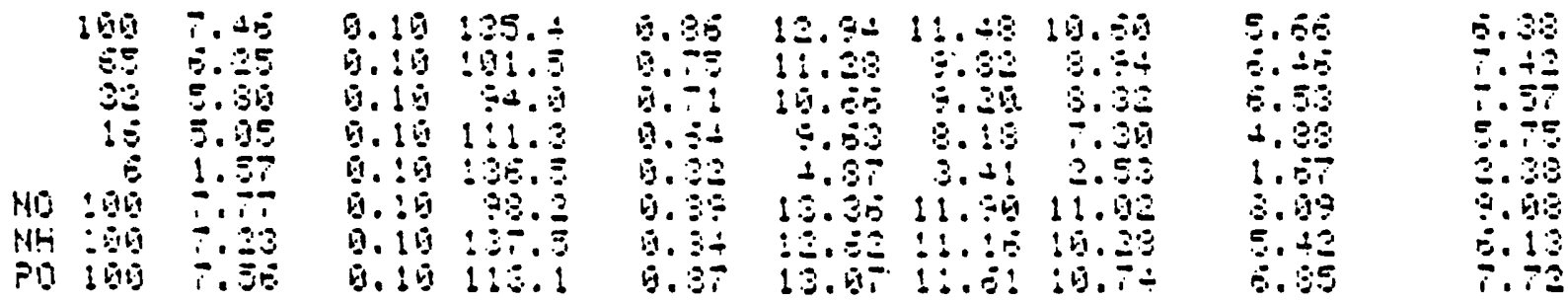


QUPATILO

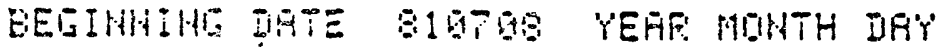

SUHFISE SOE SUHSET OGOL IH MILITAPY HOURE

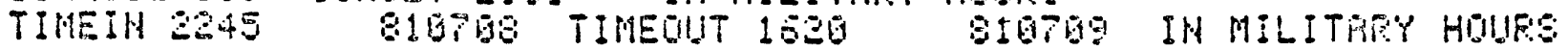

OUEFHISIT IMCUEATIOH

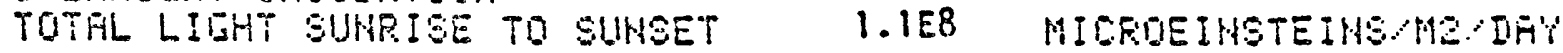

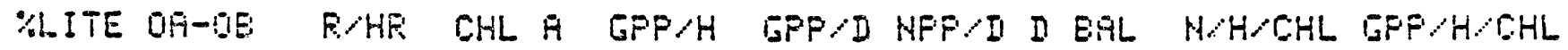

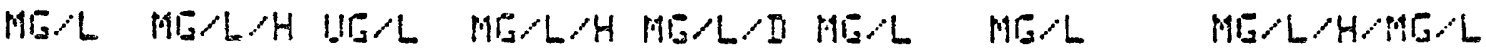

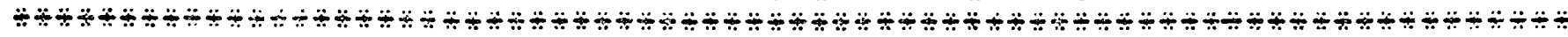

\begin{tabular}{|c|c|c|c|c|c|c|c|c|}
\hline $\begin{array}{r}106 \\
65 \\
35 \\
16 \\
6 \\
100 \\
106 \\
106\end{array}$ & 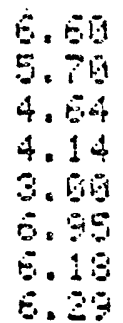 & 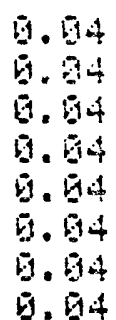 & $\begin{array}{l}55.7 \\
58.3 \\
78.8 \\
60.6 \\
87.5 \\
45.5 \\
57.5 \\
40.5\end{array}$ & 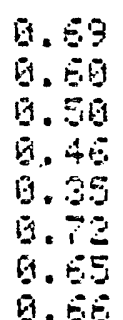 & $\begin{array}{r}19.28 \\
8.98 \\
7.58 \\
5.80 \\
5.28 \\
19.72 \\
9.64 \\
9.80\end{array}$ & $\begin{array}{r}9.59 \\
3.34 \\
6.07 \\
6.17 \\
4.58 \\
10.60 \\
9.61 \\
9.10\end{array}$ & 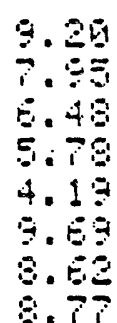 & $\begin{array}{r}11.58 \\
9.8 \\
5.35 \\
5.15 \\
3.58 \\
14.91 \\
19.58 \\
15.35\end{array}$ \\
\hline
\end{tabular}

QUHATICO

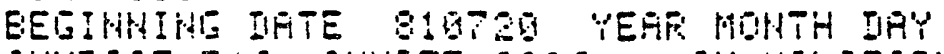

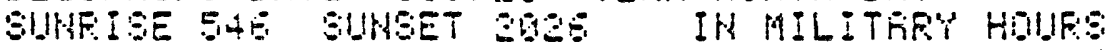

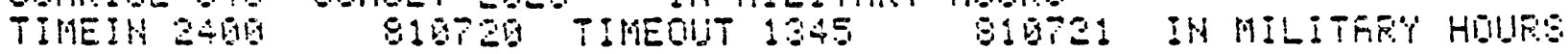

QUEFHIGHT IMBIEATIOH

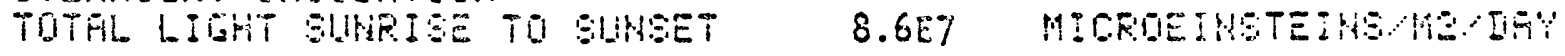

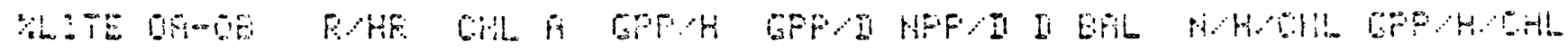

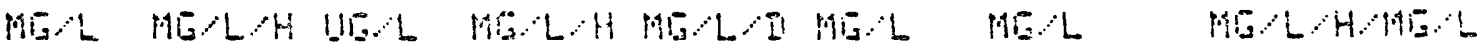

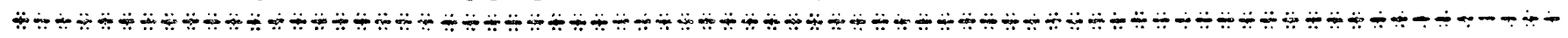

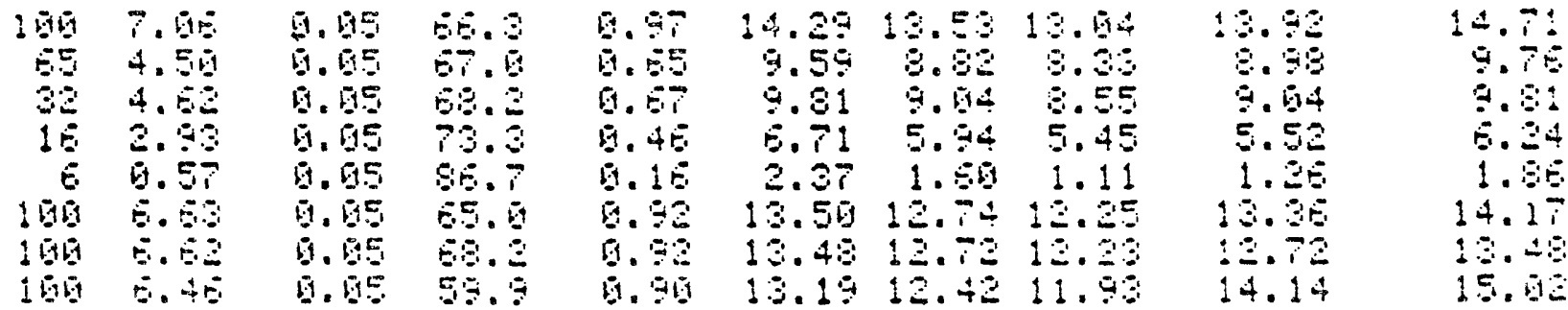

